\title{
Characters on infinite groups and rigidity
}

\author{
Dissertation \\ zur Erlangung des mathematisch-naturwissenschaftlichen Doktorgrades \\ "Doctor rerum naturalium" \\ der Georg-August-Universität Göttingen \\ im Promotionsprogramm Mathematical Science \\ der Georg-August University School of Science (GAUSS) \\ vorgelegt von \\ Rahel Brugger \\ aus Freiburg im Breisgau. \\ Göttingen, 2018
}




\section{Betreuungsausschuss}

Erstbetreuer: Prof. Dr. Thomas Schick,

Mathematisches Institut,

Georg-August Universität Göttingen

Zweitbetreuer: Prof. Dr. Ralf Meyer,

Mathematisches Institut,

Georg-August Universität Göttingen

Drittbetreuer: Dr. Vadim Alekseev,

Institut für Geometrie,

Technische Universität Dresden

Mitglieder der Prüfungskommission

Referent: Prof. Dr. Thomas Schick,

Mathematisches Institut, Georg-August Universität Göttingen

Korreferent: Prof. Dr. Ralf Meyer,

Mathematisches Institut,

Georg-August Universität Göttingen

Weitere Mitglieder der Prüfungskommission

Dr. Vadim Alekseev,

Institut für Geometrie, Technische Universität Dresden

Prof. Dr. Dorothea Bahns,

Mathematisches Institut, Georg-August Universität Göttingen

Prof. Dr. Karl-Henning Rehren,

Institut für Theoretische Physik, Georg-August Universität Göttingen

Prof. Dr. Anja Sturm,

Institut für Mathematische Stochastik, Georg-August Universität Göttingen

Tag der mündlichen Prüfung: 07.02.2018 


\begin{abstract}
We show that for a strong extension of discrete measured groupoids $1 \rightarrow \mathcal{S} \rightarrow$ $\mathcal{G} \rightarrow \mathcal{Q} \rightarrow 1$ with $L \mathcal{G}$ a finite factor, $\mathcal{Q}$ has poperty (T) if and only if the inclusion of $L \mathcal{S}$ into $L \mathcal{G}$ is corigid. In particular, this implies that $\mathcal{G}$ has property (T) if and only if $L^{\infty}(X) \subset L \mathcal{G}$ is corigid.

Furthermore, we give the definition of an invariant random positive definite function on a discrete group, generalizing both the notion of an Invariant Random Subgroup and a character. We use von Neumann algebras to show that all invariant random positive definite functions on groups with infinite conjugacy classes which integrate to the regular character are constant.

We also show a rigidity result for subfactors that are normalized by a representation of a lattice $\Gamma$ in a higher rank simple Lie group with trivial center into a finite factor. This implies that every subfactor of $L \Gamma$ which is normalized by the natural copy of $\Gamma$ is trivial or of finite index.
\end{abstract}





\section{Acknowledgements}

My primary thanks go to Vadim Alekseev for giving me such interesting questions and being a great advisor. I also would like to thank my other advisors Thomas Schick and Ralf Meyer for their support. Furthermore I want to thank Jesse Peterson for very useful comments on earlier versions of parts of this work, Miklós Abért and Andreas Thom for inventing the definition of invariant random positive definite functions together with Vadim and letting me work on it and Claire Anantharaman-Delaroche and Andreas Thom for helpful conversations.

This thesis was supportet by the German Research Foundation (DFG) through the Research Training Group "Mathematical structures in modern quantum physics". The research about invariant random positive definite functions was initiated at the Erwin Schrödinger Institute programme "measured group theory", February 2016. Correspondingly, I would like to thank the Erwin Schrödinger Institute for the nice working atmosphere during the workshop. 


\section{Contents}

1 Introduction 4

1.1 Outline of results . . . . . . . . . . . . . . . . 4

1.2 Preliminaries on von Neumann algebras. . . . . . . . . . . . 8

1.2 .1 Topologies on von Neumann algebras . . . . . . . . . . 8

1.2 .2 Factors $\ldots \ldots \ldots \ldots \ldots \ldots$

$1.2 .3 \quad$ Finite von Neumann algebras . . . . . . . . . . . . . 10

1.2 .4 Conditional expectations . . . . . . . . . . . . . . 13

$1.2 .5 \quad$ Affiliated operators . . . . . . . . . . . . . . . 14

1.3 Preliminaries on ergodic theory $\ldots \ldots \ldots \ldots \ldots \ldots$

1.3 .1 Group actions and equivalence relations on measure spaces 14

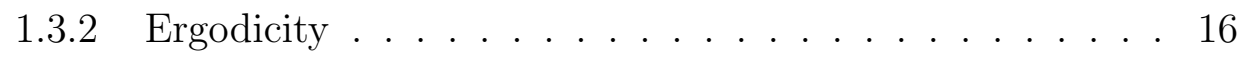

2 Property (T) for discrete measured groupoids and their von $\begin{array}{ll}\text { Neumann algebras } & 19\end{array}$

2.1 Discrete measured groupoids and their von Neumann algebras . 19

2.2 Representations and bimodules $\ldots \ldots \ldots \ldots . \ldots . \ldots 22$

$2.3 \quad$ Almost invariant sections and property (T) for groupoids . . . . 24

2.4 Almost invariant sections and almost central vectors. . . . . . . 28

2.5 Corigidity and rigidity $\ldots \ldots \ldots \ldots$

2.6 Strong extensions $\ldots \ldots \ldots \ldots \ldots$

2.7 Property ( $\mathrm{T}$ ) for groupoids and corigidity . . . . . . . . . . . 32

2.8 Rigidity for groupoid von Neumann algebras . . . . . . . . . 36

3 Disintegration rigidity for invariant random positive definite functions 38

$3.1 \quad$ Characters on discrete groups $\ldots \ldots \ldots$. . . . . . . . . . . 38

3.2 Invariant Random Subgroups . . . . . . . . . . . . . . . . 40

3.3 Definition and examples of invariant random positive definite

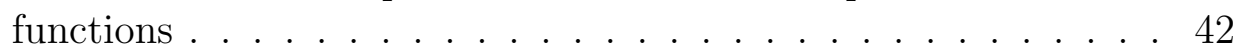

$3.4 \quad$ Invariant random positive definite functions and von Neumann

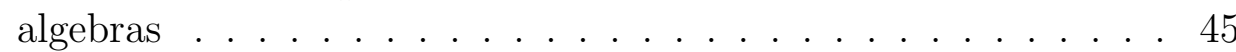

$3.5 \quad$ Disintegration rigidity of the regular character on i.c.c. groups . 50 
4 A rigidity result for regular subfactors 53

4.1 A question about regular subfactors of the von Neumann algebra

of lattices in higher-rank groups . . . . . . . . . . . . . . . 53

4.2 Peterson machine with coefficients in $N \ldots$. . . . . . . . 54

4.3 On the relative commutant . . . . . . . . . . . . . . . . . . 60

\begin{tabular}{ll}
\hline Bibliography & 63
\end{tabular} 


\section{Chapter 1}

\section{Introduction}

\subsection{Outline of results}

In this thesis we collect results in different topics in von Neumann algebras and measured group theory. Besides the introduction there are three chapters.

In Chapter 2 we discuss generalizations of the following definition that was given by Kazhdan in 1967 in order to show that certain groups are finitely generated.

Definition 1.1.1. A discrete group has property $(T)$ if every unitary representation $\pi: \Gamma \rightarrow U(H)$ which has almost invariant vectors, i.e., a net of norm one vectors $\left(\xi_{i}\right) \subset H$ such that $\left\|\pi(\gamma) \xi_{i}-\xi_{i}\right\| \rightarrow 0$ for all $\gamma \in \Gamma$, has an invariant vector, i.e., a nonzero vector $\xi \in H$ such that $\pi(\gamma) \xi=\xi$ for all $\gamma \in \Gamma$.

Typical examples of groups with property $(\mathrm{T})$ are lattices in higher rank simple Lie groups, for example, $\operatorname{SL}(n, \mathbb{Z})$ for $n \geq 3$. The definition has since then proven to be useful in many contexts, for example, in the proof of Margulis' normal subgroup theorem or in proving that the Lebesgue measure is the unique rotation invariant measure defined on all Lebesgue-measurable subsets of the unit sphere $S^{n}$ for $n \geq 2$.

The notion of property $(\mathrm{T})$ has been generalized to finite von Neumann algebras in CJ85] by Alain Connes and Vaughan Jones. In their definition representations of the group are replaced by bimodules of the von Neumann algebra and (almost) invariant vectors are replaced by (almost) central vectors in the bimodule. Robert Zimmer has defined property $(\mathrm{T})$ for group actions of discrete groups and discrete measured equivalence relations in [Zim81], which was generalized to discrete measured groupoids by Claire Anantharaman-Delaroche in [AD05]. Representations of measured groupoids act on bundles over the unit space of the groupoid and property $(\mathrm{T})$ is defined via (almost) invariant sections in these bundles.

As one can associate a finite von Neumann algebra to a discrete measured groupoid, the question about connections between property $(\mathrm{T})$ for the 
groupoid and property $(\mathrm{T})$ for its von Neumann algebra arises. It turns out that if $\mathcal{G}$ is a discrete measured groupoid such that the corresponding von Neumann algebra $L \mathcal{G}$ is a finite factor, then $\mathcal{G}$ has property ( $\mathrm{T}$ ) if and only if the inclusion $L^{\infty}(X) \subset L \mathcal{G}$ is corigid, some kind of relative property (T) defined by Sorin Popa and C. Anantharaman-Delaroche.

While working on this text we were informed that this equivalence has been proven by Martino Lupini this year [Lup17, Theorem 3.5]. For discrete equivalence relations the result had been stated before several times. However, we could not find an earlier proof. In particular, the version of Pop86 we know does not contain Section 4.8, which is cited in Pop06. Things become more complicated by the fact that there have been several definitions similar to corigidity that are not known to be equivalent. Moo82] states the equivalence of a discrete measured equivalence relation having property $(\mathrm{T})$ to such a slightly different notion, which is probably wrong.

We give a proof independent of [Lup17, Theorem 3.5] of the above equivalence in the more general setting of strong extensions introduced by Roman Sauer and Andreas Thom in [ST10].

Theorem 1.1.2 (Theorem 2.7.1). Let

$$
1 \rightarrow \mathcal{S} \rightarrow \mathcal{G} \rightarrow \mathcal{Q} \rightarrow 1
$$

be a strong extension of discrete measured groupoids such that $L \mathcal{G}$ is a finite factor. Then $\mathcal{Q}$ has property $(T)$ if and only if $L \mathcal{S} \subset L \mathcal{G}$ is corigid.

On the way, we discuss different possible definitions of property $(\mathrm{T})$ for discrete measured groupoids, which arise from different ways to define almost invariant sections for a representation of a groupoid. We show that they give equivialent characterizations of property $(\mathrm{T})$.

Having answered the question how property $(\mathrm{T})$ of $\mathcal{G}$ is characterized in terms of von Neumann algebras, one could also ask how property (T) of $L \mathcal{G}$ is characterized in terms of the groupoid. We do not answer this question, but draw the attention to results of Adrian Ioana, who gave a sufficient condition in terms of the groupoid that implies that the inclusion $L \mathcal{G}^{(0)} \subset L \mathcal{G}$ is rigid, a notion of S. Popa, which is complementary to corigidity in the sense that an inclusion $N \subset M$ is both rigid and corigid if and only if $M$ has property (T). Together with our result about corigidity this gives a condition in terms of the groupoid that implies that $L \mathcal{G}$ has property $(\mathrm{T})$. In the case of group actions this condition is also necessary.

In the last years there has been a lot of progress around the definition of an Invariant Random Subgroup (IRS), which shifted the attention in the study of ergodic group actions from their orbit equivalence relations to their stabilizers. We define a generalization of Invariant Random Subgroups, which we call invariant random positive definite functions, and study them in Chapter 3 . 
Definition 1.1.3 (Definition 3.3.1). Let $\Gamma$ be a discrete group. An invariant random positive definite function (i.r.p.d.f. ) is a measurable $\Gamma$-equivariant map

$$
\varphi: \Omega \rightarrow \mathrm{PD}(\Gamma)
$$

where $(\Omega, \mu)$ is a standard probabilitiy space with a measure preserving $\Gamma$ action, and $\mathrm{PD}(\Gamma)$ are the normalized positive definite functions $\phi$ on $\Gamma$ with $\Gamma$-action given by $(g . \phi)(h)=\phi\left(g^{-1} h g\right)$ for $\phi \in \operatorname{PD}(\Gamma)$ and $g, h \in \Gamma$.

This specializes to the definition of an IRS if we demand each $\varphi(\omega)$ to be the characteristic function of the stibilizer subgroup of $\omega$.

As an example for an i.r.p.d.f. let $(\Omega, \mu)=(S, \lambda)$ be the unit sphere in $\mathbb{C}^{n}$ with Lebesgue measure and $\Gamma$ a discrete subgroup of the unitary group $U(n)$, acting on $S$ in the natural way. Then

$$
\varphi: S \rightarrow \operatorname{PD}(\Gamma), \quad \varphi_{\xi}(\gamma)=\langle\gamma \xi, \xi\rangle \quad \forall \xi \in S, \gamma \in \Gamma
$$

is an invariant random positive definite function.

This definition is also closely related to the notion of a character on $\Gamma$, i.e. a conjugation invariant normalized positive definite function. Indeed, if $\varphi$ is an i.r.p.d.f. ,

$$
\mathbb{E}[\varphi]:=\int_{\Omega} \varphi(\omega) d \omega
$$

is a character.

A construction of Anatoly Vershik shows that in the case of $\Gamma=S_{\infty}$ every extremal character, except for the regular, the trivial and the alternating character, is of this form for a non-constant i.r.p.d.f. $\varphi$. Some of these i.r.p.d.f.'s are IRSes, some are "twisted IRSes" arising from cocyles of the action.

Characters and i.r.p.d.f.'s share the property that every character and every i.r.p.d.f. can be decomposed into extremal ones, but, in contrast to the situation for characters, this decomposition is not unique in the case of i.r.p.d.f.'s.

The main result of Chapter 3 is the following theorem.

Theorem 1.1.4 (Theorem 3.5.1). Let $\Gamma$ be a group where every nontrivial conjugacy class is infinite and let $\varphi: \Omega \rightarrow \mathrm{PD}(\Gamma)$ be an i.r.p.d.f. on $\Gamma$ with $\mathbb{E}[\varphi]=\delta_{e}$. Then $\varphi(\omega)=\delta_{e}$ for almost every $\omega \in \Omega$.

We call this phenomenon disintegration rigidity of the regular character $\delta_{e} \in \mathrm{Ch}(\Gamma)$.

$\Gamma$ having infinite conjugacy classes is equivalent to $\delta_{e} \in \operatorname{Ch}(\Gamma)$ being an extremal character. Hence the theorem states disintegration rigidity of $\delta_{e}$ in all cases where it has a chance to be disintegration rigid.

The main step in the proof of this theorem is to translate a given ergodic i.r.p.d.f. $\varphi$ with $\mathbb{E}[\varphi]=\delta_{e}$ into a random variable $f: \Omega \rightarrow L^{1}(L \Gamma)$ which fulfills the invariance condition $f(\gamma \cdot \omega)=\pi\left(\gamma^{-1}\right) f(\omega) \pi(\gamma)$. We then show that such a 
function must be constantly 1, using that the conjugation action of $\Gamma$ on $L \Gamma$ is weakly mixing. Then $\varphi$ also must be constant. This method might also apply to other characters than the regular one.

In Chapter 4 we study the following question.

Question 1.1.5 (Question 4.1.4). Is every regular subfactors of the group von Neumann algebra of a lattice in a higher-rank simple Lie group with trivial center trivial or of finite index?

The reason to hope for such a rigidity phenomenon is that this would be analogous to Margulis' Normal Subgroup Theorem, which states that for such groups every normal subgroup is trivial or of finite index.

In fact, we only look at the special case where a subfactor $N \subset L \Gamma$ is actually normalized by a unitary representation $\pi: \Gamma \rightarrow U(L \Gamma)$ such that $N$ and $\pi(\Gamma)$ generate $M$. We use methods developed by Jesse Peterson for the proof of his character rigidity theorem to prove the following theorem.

Theorem 1.1.6 (Theorem 4.2.7). Let $\Gamma$ be a lattice in a simple real Lie group $G$ which has trivial center and real rank at least 2. Let $M$ be a finite factor, $N \subset M$ a subfactor and $\pi: \Gamma \rightarrow \mathcal{N}_{M}(N)$ a unitary representation of $\Gamma$ into the normalizer of $N$ such that the action $\Gamma \curvearrowright M$ given by $\alpha_{\gamma}(x)=\pi(\gamma) x \pi\left(\gamma^{-1}\right)$ is ergodic and $M=(N \cup \pi(\Gamma))^{\prime \prime}$. If $M \neq N \rtimes \Gamma$ and $N^{\prime} \cap M$ is finite-dimensional, then $[M: N]<\infty$.

Peterson's proof is inspired by Margulis' proof in the sense that the proof of the normal subgroup theorem is based on the fact that an amenable discrete group with property $(\mathrm{T})$ is finite, whereas the proof of character rigidity is based on the fact that an amenable factor with property ( $\mathrm{T}$ ) is finitedimensional.

We adjust Peterson's proof to the situation of subfactors described above by putting coefficients in $N$ into it. Then we use that if an inclusion $N \subset M$ is both amenable and corigid and the relative commutant is finite-dimensional, the inclusion is of finite index.

In the case where $M=L \Gamma$ and $\pi$ is the left regular representation we get rid of the assumption that the relative commutant is finite-dimensional. To do so, we construct a projective representation into the relative commutant and use that Peterson's proof of character rigidity can be slightly modified to give a rigidity result for projective representations. We get the following theorem.

Theorem 1.1.7 (Theorem 4.3.1). Let $\Gamma$ be a lattice in a simple real Lie group $G$ which has trivial center and real rank at least 2 . Let $N \subset L \Gamma$ be a subfactor which is normalized by the natural copy of $\Gamma$ in $L \Gamma$. Then $[M: N]<\infty$ or $N=\mathbb{C}$. 


\subsection{Preliminaries on von Neumann algebras}

\subsubsection{Topologies on von Neumann algebras}

A reference for this and the following subsection is Bla06].

Definition 1.2.1. A von Neumann algebra is a ${ }^{*}$-subalgebra $M \subset B(H)$ of the bounded operators on some Hilbert space that contains the identity and is closed in the weak operator topology (WOT).

The weak operator topology is not independent of the embedding of $M$ into some $B(H)$. Therefore, a more natural topology for von Neumann algebras is the ultraweak topology.

Definition 1.2.2. $\quad$ - The ultraweak (or $\sigma$-weak or weak*) topology on a von Neumann algebra $M \subset B(H)$ is the restriction of WOT on $M \bar{\otimes} B\left(\ell^{2}\right)$ (the smallest von Neumann algebra containing the algebraic tensor product) to $M$.

- The ultrastrong (or $\sigma$-strong) topology is the restriction of the strong operator topology (SOT) on $M \bar{\otimes} B\left(\ell^{2}\right)$ to $M$.

- The strong* topology is the topology induced by the seminorms $\|T\|_{\xi}=$ $\|T \xi\|+\left\|T^{*} \xi\right\|$ for $\xi \in H$.

Theorem 1.2.3. WOT and SOT coincide on convex sets. The ultraweak topology and WOT as well as the ultrastrong topology and SOT coincide on bounded sets.

Definition 1.2.4. A positive map $\Phi: M \rightarrow N$ between von Neumann algebras is called normal if $\Phi\left(\sup x_{i}\right)=\sup \Phi\left(x_{i}\right)$ for all norm bounded, increasing nets $\left(x_{i}\right) \subset M_{+}$.

A bounded linear functional is normal if it is ultraweakly (or ultrastrongly) continuous. The set of normal linear functionals on $M$ is denoted by $M_{*}$.

The notation $M_{*}$ comes from the fact that $M$ is the dual space of $M_{*}$. For positive linear functionals the two characterizations of normality in Definition 1.2.4 coincide. Also *-homomorphisms are normal if and only if they are ultraweakly (or ultrastrongly) continuous. *-isomorphisms between von Neumann algebras are automatically normal. We write $\operatorname{Aut}(M)$ for the ${ }^{*}$-isomorphisms on $M$.

Definition 1.2.5. A von Neumann algebra $M$ is called separable if $M_{*}$ is separable in the norm topology.

$M$ is separable if and only if it is isomorphic to a von Neumann subalgebra of some $B(H)$ for a separable Hilbert space $H$. 
Theorem 1.2.6. The double commutant

$$
A^{\prime \prime}=\{x \in B(H) \mid[y, a]=0 \forall a \in A \Rightarrow[x, y]=0\}
$$

of any selfadjoint subset $A \subset B(H)$ is equal to the WOT-closure of the subalgebra generated by $A$ and therefore a von Neumann algebra.

Example 1.2.7. For a discrete group $\Gamma$ we define the group von Neumann algebra

$$
L \Gamma=\{\lambda(g) \mid g \in \Gamma\}^{\prime \prime} \subset B\left(\ell^{2}(\Gamma)\right)
$$

where $\lambda(g) \in U\left(\ell^{2}(\Gamma)\right)$ is the operator that sends a basis element $\delta_{h}$ to $\delta_{g h}$.

$\lambda$ is called the left regular representation.

Example 1.2.8. Let $c: \Gamma \times \Gamma \rightarrow S^{1}$ be a 2-cocycle on $\Gamma$, i.e.,

$$
c\left(\gamma_{1} \gamma_{2}, \gamma_{3}\right) c\left(\gamma_{1}, \gamma_{2}\right)=c\left(\gamma_{1}, \gamma_{2} \gamma_{3}\right) c\left(\gamma_{2}, \gamma_{3}\right) \quad \forall \gamma_{1}, \gamma_{2}, \gamma_{3} \in \Gamma
$$

and $c(\gamma, e)=c(e, \gamma)=1$ for all $\gamma \in \Gamma$. Then the twisted left regular representation with cocycle $c$ is the projective representation given by

$$
\lambda_{c}(\gamma): \ell^{2}(\Gamma) \rightarrow \ell^{2}(\Gamma), \quad \lambda_{c}(\gamma) \delta_{g}=c(\gamma, g) \delta_{\gamma g}
$$

and $L \Gamma_{c}=\lambda_{c}(\Gamma)^{\prime \prime} \subset B\left(\ell^{2}(\Gamma)\right)$ is called the twisted group von Neumann algebra with cocycle $c$.

Example 1.2.9. If $M \subset B(H)$ is a von Neumann algebra and $\alpha: \Gamma \rightarrow \operatorname{Aut}(M)$ an action of a discrete group $\Gamma$ on $M$, we can define the crossed product

$$
M \rtimes_{\alpha} \Gamma \subset B\left(H \otimes \ell^{2}(\Gamma)\right)
$$

as the von Neumann algebra which is generated by the operators $1 \otimes \lambda(\gamma)$ for $\gamma \in \Gamma$ and $\lambda$ the left regular representation and the operators $\iota(x) \in$ $B\left(H \otimes \ell^{2}(\Gamma)\right)$ with $x \in M$ given by

$$
\iota(x)\left(\delta_{\gamma} \otimes \xi\right)=\alpha_{\gamma^{-1}}(x)(\xi) \otimes \delta_{\gamma} .
$$

Conjugation with $1 \otimes \lambda$ implements the action $\alpha$, i.e., for all $x \in M$ and $\gamma \in \Gamma$

$$
(1 \otimes \lambda)(\gamma) \iota(x)(1 \otimes \lambda)\left(\gamma^{-1}\right)=\iota\left(\alpha_{\gamma}(x)\right) .
$$

Theorem 1.2.10 (Kaplansky density theorem, [Tak02, Theorem II.4.8]). Let $A \subset B(H)$ be a selfadjoint subalgebra and let $M=A^{\prime \prime}$. Then the unit ball of $A$ is strongly* dense in the unit ball of $M$. 


\subsubsection{Factors}

Definition 1.2.11. A von Neumann algebra is called a factor if its center contains only the multiples of the identity.

Example 1.2.12. $L \Gamma$ is a factor if and only if all nontrivial conjugacy classes of $\Gamma$ are infinite. One says then that $\Gamma$ is i.c.c..

Definition 1.2.13. If $(\Omega, \mu)$ is a measure space and $\left(H_{\omega}\right)_{\omega \in \Omega}$ is a measurable field of Hilbert spaces, the direct integral

$$
\int_{\Omega}^{\oplus} H_{\omega} d \mu(\omega)
$$

is the Hilbert space consisisting of $L^{2}$-sections, i.e., $\left(\xi_{\omega}\right)_{\omega \in \Omega}$ with $\xi_{\omega} \in H_{\omega}$ such that $\omega \mapsto\left\|\xi_{\omega}\right\|$ is in $L^{2}(\Omega, \mu)$, with scalar product

$$
\left\langle\left(\xi_{\omega}\right)_{\omega \in \Omega},\left(\eta_{\omega}\right)_{\omega \in \Omega}\right\rangle=\int_{\Omega}\left\langle\xi_{\omega}, \eta_{\omega}\right\rangle d \mu(\omega)
$$

If $M_{\omega} \subset B\left(H_{\omega}\right)$ is a measurable field of von Neumann algebras, $\int_{\Omega}^{\oplus} x_{\omega} d \mu(\omega)$ with $x_{\omega} \in M_{\omega}$ is the operator on $\int_{\Omega}^{\oplus} H_{\omega} d \mu(\omega)$ defined by

$$
\int_{\Omega}^{\oplus} x_{\omega} d \mu(\omega)\left(\xi_{\omega}\right)_{\omega \in \Omega}=\left(x_{\omega} \xi_{\omega}\right)_{\omega \in \Omega}
$$

and the direct integral of von Neumann algebras $\int_{\Omega}^{\oplus} M_{\omega} d \mu(\omega)$ is the von Neumann algebra consisting of all bounded operators of this form such that for all $\xi, \eta \in \int_{\Omega}^{\oplus} H_{\omega} d \mu(\omega)$ the map $\omega \mapsto\left\langle x_{\omega} \xi_{\omega}, \eta_{\omega}\right\rangle$ is measurable. Everything in this construction is modulo sets of measure zero.

Often it is enough to study factors because every separable von Neumann algebra can be decomposed into a direct integral of factors.

Theorem 1.2.14. Every separable von Neumann algebra $M$ is isomorphic to a direct integral $\int_{\Omega} M_{\omega} d \mu(\omega)$ of factors $M_{\omega}$ such that the center $\mathcal{Z}(M)$ of $M$ is isomorphic to $L^{\infty}(\Omega, \mu)$.

\subsubsection{Finite von Neumann algebras}

A reference for this subsection is [ADP10.

Definition 1.2.15. A von Neumann algebra $M$ is finite if there is a linear map $\tau: M \rightarrow \mathbb{C}$ such that

i) $\tau\left(x^{*} x\right)=0 \Leftrightarrow x=0$ ( $\tau$ is faithful $)$,

ii) $\tau$ is normal, 
iii) $\tau(x y)=\tau(y x)$ for all $x, y \in M(\tau$ is tracial $)$ and

iv) $\tau(1)=1$ and $\tau\left(x^{*} x\right) \geq 0$ for all $x \in M$ ( $\tau$ is a state $)$.

$\tau$ is called a trace on $M$.

Example 1.2.16. The von Neumann algebra of bounded operators on a Hilbert space is finite if and only if the Hilbert space is finite-dimensional.

Example 1.2.17. $L \Gamma$ and $L \Gamma_{c}$ are finite with trace $\tau(x)=\left\langle x \delta_{e}, \delta_{e}\right\rangle$.

Example 1.2.18. If $M$ is a finite von Neumann algebra with trace $\tau_{M}$ and $\alpha: \Gamma \rightarrow \operatorname{Aut}(M)$ is a trace-preserving action, then $M \rtimes_{\alpha} \Gamma$ is a finite von Neumann algebra with trace defined by

$$
\tau((1 \otimes \lambda(\gamma)) \iota(x))=\delta_{e}(\gamma) \tau_{M}(x) .
$$

Theorem 1.2.19. The trace on a finite factor is unique.

Theorem 1.2.20. A von Neumann algebra is finite if and only if $v^{*} v=1$ implies $v v^{*}=1$ for all $v \in M$.

Theorem 1.2.21. If $p$ and $q$ are projections in a finite von Neumann algebra $(M, \tau), \tau(p)=\tau(q)$ if and only if there exists $a v \in M$ such that $p=v^{*} v$ and $q=v v^{*}$.

If $(M, \tau)$ is a finite von Neumann algebra, we can build a Hilbert space $L^{2}(M, \tau)$ as the completion of $M$ with respect to the norm $\|x\|_{2}=\sqrt{\tau\left(x^{*} x\right)}$. If we view an element $x \in M$ as an element of $L^{2}(M, \tau)$, we sometimes write $\hat{x} \in L^{2}(M, \tau)$. On this dense subset the scalar product of $L^{2}(M, \tau)$ is $\langle\hat{x}, \hat{y}\rangle=$ $\tau\left(x y^{*}\right)$.

$M$ can also be viewed as a subset of $B\left(L^{2}(M, \tau)\right)$ as for every $x \in M$ the map $\hat{y} \mapsto \hat{x y}$ extends to a bounded operator on $L^{2}(M, \tau)$. This homomorphism $M \rightarrow B\left(L^{2}(M, \tau)\right)$ is called the standard representation of $M$.

Example 1.2.22. We have $L^{2}\left(L \Gamma_{c}, \tau\right)=L^{2}(L \Gamma, \tau)=\ell^{2}(\Gamma)$ and $L^{2}(M \rtimes \Gamma, \tau)=$ $L^{2}\left(M,\left.\tau\right|_{M}\right) \otimes \ell^{2}(\Gamma)$ for the traces defined above.

Proposition 1.2.23. The map $\hat{x} \mapsto \hat{x}^{*}$ extends to an isometric, conjugatelinear operator $J$ on $L^{2}(M, \tau)$ such that $J^{2}=1, J M J=M^{\prime} \subset B\left(L^{2}(M, \tau)\right)$ and $J M^{\prime} J=M$.

\section{Dimension of Hilbert modules over finite von Neumann algebras}

For a finite von Neumann algebra $M$ with fixed trace $\tau$ we have a dimension function $\operatorname{dim}_{M}$ on Hilbert modules over $M$.

Definition 1.2.24. Let $(M, \tau)$ be a finite von Neumann algebra. A Hilbert module over $M$ is a Hilbert space $H$ with a left action of $M$ such that $1 \in M$ acts identically. $H$ is called finitely generated if it is isomorphic to a submodule of $\bigoplus_{1}^{n} L^{2}(M, \tau)$. 
Definition 1.2.25. Let $H$ be a finitely generated Hilbert module over $M$ with $H \subset \bigoplus_{1}^{n} L^{2}(M, \tau)$. Let $\Phi$ be the orthogonal projection in $B\left(\bigoplus_{1}^{n} L^{2}(M, \tau)\right)$ on $H$. Then it is given by a matrix $\left[\Phi_{i, j}\right]_{i, j=1, \ldots, n}$, where $\Phi_{i, j}: L^{2}(M) \rightarrow L^{2}(M)$ are bounded linear maps. The $\Phi_{i, j}$ commute with the left $M$-action, hence by Proposition 1.2 .23 each $\Phi_{i, j}$ is given by right multiplication with some element in $M$, which we again denote by $\Phi_{i, j}$. Then the dimension of $H$ over $M$ is defined as

$$
\operatorname{dim}_{M}(H):=\sum_{i=1}^{n} \tau\left(\Phi_{i, i}\right) .
$$

If $H$ is not finitely generated, we define

$$
\operatorname{dim}_{M}(H):=\sup \left\{\operatorname{dim}_{M}(V) \mid V \subset H \text { finitely generated }\right\} .
$$

Remark 1.2.26. This construction is independent of the chosen embedding.

An inclusion of finite von Neumann algebras $N \subset M$ is an inclusion of von Neumann algebras with fixed traces such that the trace of $N$ is the restriction of the trace of $M$. We will need the following facts.

Lemma 1.2.27. i) If $N \subset M$ is an inclusion of finite von Neumann algebras, then $\operatorname{dim}_{N}(H) \leq \operatorname{dim}_{M}(H)$ for every Hilbert $M$-module $H$.

ii) If $N \subset M$ is an inclusion of finite von Neumann algebras such that $N$ is finite-dimensional and $\operatorname{dim}_{N}\left(L^{2}(M)\right)<\infty$, then $M$ is also finitedimensional.

iii) If $N_{i} \subset M_{i}$ are inclusions of finite von Neumann algebras, then

$$
\operatorname{dim}_{N_{1} \bar{\otimes} N_{2}}\left(L^{2}\left(M_{1} \bar{\otimes} M_{2}, \tau_{1} \otimes \tau_{2}\right)\right)=\operatorname{dim}_{N_{1}}\left(L^{2}\left(M_{1}, \tau_{1}\right)\right) \cdot \operatorname{dim}_{N_{2}}\left(L^{2}\left(M_{2}, \tau_{2}\right)\right) .
$$

iv) If $N \subset M$ is an inclusion of finite von Neumann algebras and $M \subset$ $\sum_{x \in F} N x$ for a finite set $F \subset M$, then $\operatorname{dim}_{N}\left(L^{2}(M)\right)<\infty$.

v) If $N \subset M$ is an inclusion of finite von Neumann algebras and $M$ contains infinitely many $x \in M$ such that the sets $N x$ are pairwise orthogonal in $L^{2}(M)$, then $\operatorname{dim}_{N}\left(L^{2}(M)\right)=\infty$.

Definition 1.2.28. The index of an inclusion $N \subset M$ of finite factors is defined as

$$
[M: N]=\operatorname{dim}_{N}\left(L^{2}(M)\right)
$$

This depends only on the factors themselves since the traces are unique. 


\section{Types of von Neumann algebras}

Definition 1.2.29. $\quad$ i) A von Neumann algebra is of type $I$ if almost every factor in its direct integral decomposition is $B(H)$ for some Hilbert space $H$. It is of type $I_{1}$ if almost every factor in its direct integral decomposition is a matrix algebra $\mathbb{M}_{n}(\mathbb{C})$.

ii) A von Neumann algebra is of type $I I_{1}$ if almost every factor in its direct integral decomposition is finite and not of type I.

iii) A von Neumann algebra is of type $I I_{\infty}$ if for almost every factor $M$ in its direct integral decomposition there is a net of increasing projections $\left\{p_{i}\right\} \subset M$ such that $p_{i} \rightarrow 1$ in SOT and each $p_{i} M p_{i}$ is of type $\mathrm{II}_{1}$.

iv) A von Neumann algebra is of type III if almost every factor in its direct integral decomposition is not of type I or II.

Theorem 1.2.30. A factor is of type I if and only if it has minimal projections.

\subsubsection{Conditional expectations}

A reference for this subsection is [Bla06].

Definition 1.2.31. Let $A \subset B$ be $C^{*}$-algebras. A conditional expectation $E: B \rightarrow A$ is a contractive projection, i.e., a linear map such that $E(a)=a$ for all $a \in A$ and $\|E(b)\| \leq\|b\|$ for all $b \in B$.

Theorem 1.2.32. Each conditional expectation is completely positive, i.e. the map $E \otimes \operatorname{id}_{n}: \mathbb{M}_{n}(B) \rightarrow \mathbb{M}_{n}(A)$ is positive for all $n \in \mathbb{N}$, and each conditional expectation is a bimodule map, i.e. $E\left(a b a^{\prime}\right)=a E(b) a^{\prime}$ for all $a, a^{\prime} \in A$ and $b \in B$.

Theorem 1.2.33. Let $(M, \tau)$ be a finite von Neumann algebra and $N \subset M$ a von Neumann subalgebra (i.e., $1_{M} \in N$ ). Then there is a unique tracepreserving normal conditional expectation $E: M \rightarrow N$.

A conditional expectation is normal iff it is weak* continuous.

Definition 1.2.34. A von Neumann algebra $M \subset B(H)$ is amenable if there is a conditional expectation $E: B(H) \rightarrow M$.

Amenability of $M$ is independent of the embedding $M \subset B(H)$. 


\subsubsection{Affiliated operators}

A reference for the theory of affiliated operators is Tak03].

Definition 1.2.35. If $M \subset B(H)$ is a von Neumann algebra, an unbounded, densely defined operator $x$ on $H$ is called affiliated to $M$ if, for every unitary $u$ in the commutant $M^{\prime} \subset B(H)$, the domain of $x$ is invariant under $u$ and the two operators commute. We denote the set of affiliated operators by $\mathcal{U}(M)$.

$M$ consists exactly of the bounded operators in $\mathcal{U}(M)$.

Definition 1.2.36. For a finite von Neumann algebra $(M, \tau)$ let $L^{1}(M, \tau)$ be the closure of $M$ in $\mathcal{U}(M)$ with respect to the norm $\|x\|_{1}=\tau(|x|)$.

Example 1.2.37. If $(\Omega, \mu)$ is a probability space and

$$
M=L^{\infty}(\Omega, \mu) \subset B\left(L^{2}(\Omega, \mu)\right)
$$

with trace $\tau(f)=\int_{\Omega} f d \mu$, then $\mathcal{U}(M)$ consists of all measurable functions on $\Omega$ modulo null sets and $L^{1}(M, \tau)=L^{1}(\Omega, \mu)$.

Theorem 1.2.38. Let $(M, \tau)$ be a finite von Neumann algebra.

i) $L^{1}(M)$ is an $M$-bimodule.

ii) $\tau$ extends continuously to $L^{1}(M)$.

iii) The map $L^{1}(M) \rightarrow M_{*}, x \mapsto(y \mapsto \tau(x y))$, is an isomorphism mapping positive elements to positive elements.

\subsection{Preliminaries on ergodic theory}

Our references for this section are [Gla03] and [KM04].

\subsubsection{Group actions and equivalence relations on mea- sure spaces}

Ergodic theory is about groups acting on measure spaces. We will restrict ourselves to so-called standard spaces.

Definition 1.3.1. A standard Borel space is a separable topological space which is metrizable with a complete metric together with the Borel $\sigma$-algebra generated by open sets.

Definition 1.3.2. A standard probability space is a probability space which is isomorphic as a measure space to a union of an interval (might be empty) with Borel $\sigma$-algebra and Lebesgue measure and at most countably many atoms, i.e., points with positive measure. 
Theorem 1.3.3. Every regular probability measure on a standard Borel space turns it into a standard probability space.

If $(X, \mu)$ is a standard probability space, then $L^{2}(X, \mu)$ is a separable Hilbert space.

Definition 1.3.4. Let $\Gamma \curvearrowright X$ be a group action. Then the corresponding orbit equivalence relation on $X$ is given by $x \sim y \Leftrightarrow \exists \gamma \in \Gamma: x=\gamma . y$.

Definition 1.3.5. For a Borel equivalence relation $\mathcal{R} \subset X \times X$ on a Borel space the full group $[\mathcal{R}]$ consists of all automorphisms $f \in \operatorname{Aut}(X)$ such that the graph of $f$ is contained in $\mathcal{R}$.

Definition 1.3.6. - An action $\alpha: \Gamma \curvearrowright(X, \mu)$ is called measure preserving (or $\mu$ is invariant) if $\mu \circ \alpha_{\gamma}=\mu$ for every $\gamma \in \Gamma$.

- A Borel equivalence relation $\mathcal{R} \subset X \times X$ on a measure space $(X, \mu)$ is called measure preserving if $\mu \circ f=\mu$ for every $f \in[\mathcal{R}]$.

- If $\mu$ is also a probability measure, $\alpha$ resp. $\mathcal{R}$ as above is called probability measure preserving (p.m.p.).

- An action $\alpha: \Gamma \curvearrowright(X, \mu)$ is called measure class preserving (or $\mu$ is quasiinvariant) if $\mu \circ \alpha_{\gamma} \sim \mu$ for every $\gamma \in \Gamma$, i.e., $\mu \circ \alpha_{\gamma}$ and $\mu$ have the same measure zero sets.

- A Borel equivalence relation $\mathcal{R} \subset X \times X$ on a measure space $(X, \mu)$ is measure class preserving iff $\mu \circ f \sim \mu$ for every $f \in[\mathcal{R}]$.

Clearly an action is measure (class) preserving iff its orbit equivalence relation is.

Definition 1.3.7. A measure class preserving action $\alpha: \Gamma \curvearrowright(X, \mu)$ induces a unitary representation $\alpha^{0}: \Gamma \rightarrow U\left(L^{2}(X, \mu)\right)$ given by

$$
\alpha_{\gamma}^{0}(f)(x)=f\left(\alpha_{\gamma^{-1}}(x)\right) \sqrt{\frac{d(\gamma \cdot \mu)}{d \mu}(x)},
$$

where $\frac{d \gamma \cdot \mu}{d \mu}$ denotes the Radon-Nikodym derivative. This is called the corresponding Koopman representation.

The action also induces a corresponding action on the von Neumann algebra $L^{\infty}(X, \mu)$ by $\alpha_{\gamma}(f)(x)=f\left(\alpha_{\gamma^{-1}}(x)\right)$. Note that for all $f \in L^{\infty}(\Omega, \mu) \subset$ $B\left(L^{2}(\Omega, \mu)\right)$ and all $\gamma \in \Gamma$ we have $\alpha_{\gamma}^{0} f\left(\alpha_{\gamma}^{0}\right)^{-1}=\alpha_{\gamma}(f)$.

Definition 1.3.8. An equivalence relation is called discrete if every orbit is countable and discrete. 
Definition 1.3.9. A discrete p.m.p. equivalence relation $\mathcal{R} \subset X \times X$ on $(X, \mu)$ is called amenable if there is a sequence $\lambda^{n}: \mathcal{R} \rightarrow \mathbb{R}_{\geq 0}$ of Borel functions such that

i) $\lambda_{x}^{n} \in \ell^{1}([x])$, where $\lambda_{x}^{n}(y)=\lambda^{n}(x, y)$,

ii) $\left\|\lambda_{x}^{n}\right\|_{1}=1$ and

iii) there exists an $\mathcal{R}$-invariant set $A \subset X$ of measure 1 such that

$$
\left\|\lambda_{x}^{n}-\lambda_{y}^{n}\right\|_{1} \rightarrow 0
$$

for all $x, y \in A$ with $x \mathcal{R} y$.

Definition 1.3.10. A p.m.p. equivalence relation $\mathcal{R} \subset X \times X$ is called hyperfinite if there is a sequence $\left\{\mathcal{F}_{n}\right\}_{n \in \mathbb{N}}$ of p.m.p. equivalence relations on $X$ such that $\mathcal{F}_{n} \subset \mathcal{F}_{n+1}$ for all $n$ and $\bigcup_{n \in \mathbb{N}} \mathcal{F}_{n}=\mathcal{R}$.

Theorem 1.3.11. A discrete p.m.p. equivalence relation $\mathcal{R} \subset X \times X$ on $(X, \mu)$ is amenable if and only if it is hyperfinite $\mu$-almost everywhere.

\subsubsection{Ergodicity}

Definition 1.3.12. - A group action on a measure space $\Gamma \curvearrowright(X, \mu)$ is called ergodic if every $\Gamma$-invariant, measurable subset $Y \subset X$ is null or conull, i.e., $\mu(Y)=0$ or $\mu\left(Y^{c}\right)=0$.

- An equivalence relation $\mathcal{R} \subset X \times X$ on a measure space is called ergodic if every $\mathcal{R}$-invariant, measurable set $Y$ (i.e., $x \in Y \Rightarrow[x]_{\mathcal{R}} \subset Y$ ) is null or conull.

Example 1.3.13. The action $\mathbb{Z} \curvearrowright S^{1}$ on the circle with Lebesgue measure where $n$ rotates the circle by $n$ times an angle $\alpha$ is ergodic if and only if $\alpha \notin \mathbb{Q} \pi$.

Clearly an action is ergodic iff its orbit equivalence relation is.

Remark 1.3.14. It is easy to check with a nested intervals kind of argument that an action is ergodic iff every (bounded) measurable $\Gamma$-invariant function into a standard Borel space is essentially constant.

Definition 1.3.15. An action on a von Neumann algebra $\Gamma \rightarrow \operatorname{Aut}(M)$ is called ergodic if the fixed point algebra is trivial, i.e. $M^{\Gamma}=\mathbb{C}$.

Remark 1.3.14 implies that an action $\Gamma \curvearrowright(X, \mu)$ is ergodic iff the corresponding action on $L^{\infty}(X, \mu)$ is ergodic.

Example 1.3.16. The conjugation action of an i.c.c. group $\Gamma$ on its von Neumann algebra,

$$
\alpha: \Gamma \rightarrow \operatorname{Aut}(L \Gamma), \quad \alpha_{\gamma}(x)=\lambda(\gamma) x \lambda\left(\gamma^{-1}\right)
$$

is ergodic. 
Often a non-ergodic situation can be decomposed into ergodic parts by the following theorem.

Theorem 1.3.17 (ergodic decomposition). Let $\mathcal{R} \subset X \times X$ be the orbit equivalence relation of an action $\Gamma \curvearrowright X$ of a countable group on a standard Borel space. Let $\mathcal{I}_{\mathcal{R}}$ be the set of $\mathcal{R}$-invariant probability measures on $X$ and $\mathcal{E I}_{\mathcal{R}}$ the set of ergodic $\mathcal{R}$-invariant probability measures on $X$. Then there is a unique map $\pi: X \rightarrow \mathcal{E}_{\mathcal{R}}$ with the following properties:

i) $\pi$ is $\mathcal{R}$-invariant, i.e. it maps orbits to the same measure.

ii) If $X_{\nu}:=\{x \in X \mid \pi(x)=\nu\}$, then $\nu\left(X_{\nu}\right)=1$ and $\nu$ is the unique $\mathcal{R}$-invariant measure on $\left.\mathcal{R}\right|_{X_{\nu}}$.

iii) $\mu=\int \pi(x) d \mu(x)$ for all $\mu \in \mathcal{I}_{\mathcal{R}}$.

The probability spaces $\left(X_{\nu}, \nu\right)$ are called ergodic components.

Example 1.3.18. Consider Example 1.3.13 with rational $\alpha$. Then $\pi$ maps each point to the normalized counting measure on its orbit.

We will also need the following strengthening of ergodicity:

Definition 1.3.19. A measured equivalence relation $\mathcal{R}$ is strongly ergodic if $\mu\left(C_{n} \triangle \theta\left(C_{n}\right)\right) \rightarrow 0$ for all $\theta \in[\mathcal{R}]$ implies that $\mu\left(C_{n}\right)\left(1-\mu\left(C_{n}\right)\right) \rightarrow 0$.

\section{Ergodicity of induced actions on von Neumann algebras}

We will need that the following construction preserves ergodicity. This follows Pet16].

Definition 1.3.20. Let $\Gamma \subset G$ be a closed subgroup of a locally compact group and $\theta: \Gamma \rightarrow \operatorname{Aut}(M)$ a continuous action. Pick a Borel section $s: G / \Gamma \rightarrow G$ and let $\chi: G \times G / \Gamma \rightarrow \Gamma$ be the cocycle given by $\chi(g, x)=s(g x)^{-1} g s(x)$.

Then the induced action $\tilde{\theta}$ of $G$ on $L^{\infty}(G / \Gamma) \bar{\otimes} M$, which we view as bounded functions from $G / \Gamma$ to $M$, is given by

$$
\tilde{\theta}_{g}(f)(x):=\theta_{\chi\left(g, g^{-1} x\right)} f\left(g^{-1} x\right),
$$

for $g \in G, f \in L^{\infty}(G / \Gamma) \bar{\otimes} M$ and $x \in G / \Gamma$.

Remark 1.3.21. Let $R$ be the $G$-action on $L^{\infty}(G)$ given by right multiplication. Then

$$
\Psi: L^{\infty}(G / \Gamma) \bar{\otimes} M \rightarrow\left(L^{\infty}(G) \bar{\otimes} M\right)^{(R \otimes \theta)(\Gamma)}, \quad \Psi(f)(g)=\theta_{s(\Gamma) \chi(g, g \Gamma)}(f(g \Gamma))
$$

is an isomorphism and

$$
\Psi\left(\tilde{\theta}_{g}(f)\right)=L \otimes \operatorname{id}(g) \Psi(f),
$$

where $L$ is the $G$-action on $L^{\infty}(G)$ given by left multiplication. 
Lemma 1.3.22. $\tilde{\theta}$ is ergodic iff $\theta$ is.

Proof. By Remark 1.3.21,

$$
\left(L^{\infty}(G / \Gamma) \bar{\otimes} M\right)^{\tilde{\theta}(G)} \cong\left(L^{\infty}(G) \bar{\otimes} M\right)^{(R \otimes \theta)(\Gamma) \cup(L \otimes \mathrm{id})(G)}=1 \otimes M^{\theta(\Gamma)},
$$

hence $\left(L^{\infty}(G / \Gamma) \bar{\otimes} M\right)^{\tilde{\theta}(G)}=\mathbb{C}$ if and only if $M^{\theta(\Gamma)}=\mathbb{C}$. 


\section{Chapter 2}

\section{Property (T) for discrete measured groupoids and their von Neumann algebras}

\subsection{Discrete measured groupoids and their von Neumann algebras}

In this section we collect some notation and definitions concerning discrete measured groupoids. Our references are [AD05, [ADR00] and [ST10].

Let $\mathcal{G}$ be a groupoid. We write $s$ and $r$ for the source and range maps, $\mathcal{G}_{x}$ resp. $\mathcal{G}^{x}$ for $s^{-1}(x)$ resp. $r^{-1}(x), \mathcal{G}^{(0)}$ for the units and $\mathcal{G}^{(2)}$ for the pairs of composable elements in $\mathcal{G}$. We will also often denote $\mathcal{G}^{(0)}$ by $X$. If $A \subset \mathcal{G}^{(0)}$, $\left.\mathcal{G}\right|_{A}$ is the groupoid $\{\alpha \in \mathcal{G} \mid s(\alpha), r(\alpha) \in A\}$.

A discrete measured groupoid is a groupoid $\mathcal{G}$ such that $s^{-1}(x)$ and $r^{-1}(x)$ are countable for every $x \in X, \mathcal{G}$ is a standard Borel space such that inversion, composition, range and source maps are Borel, together with a measure $\mu$ on $X$ such that the two measures on $\mathcal{G}$

$$
\begin{aligned}
& \mu_{s}(A)=\int_{X} \#\left(\mathcal{G}_{x} \cap A\right) d \mu(x) \quad \text { and } \\
& \mu_{r}(A)=\int_{X} \#\left(\mathcal{G}^{x} \cap A\right) d \mu(x)
\end{aligned}
$$

are equivalent.

A discrete measured groupoid is called p.m.p. if $(X, \mu)$ is a probability space and $\mu_{r}=\mu_{s}$. The results in this chapter will all be about discrete p.m.p. groupoids.

Example 2.1.1. If $\Gamma \curvearrowright(X, \mu)$ is a p.m.p. action of a discrete group on a standard probability space, we get a discrete p.m.p. groupoid

$$
X \rtimes \Gamma=\{(x, \gamma, y) \mid x, y \in X, \gamma \in \Gamma, g \cdot y=x\}
$$


with $s(x, \gamma, y)=y, r(x, \gamma, y)=x$, and $(x, \gamma, y)\left(y, \gamma^{\prime}, z\right)=\left(x, \gamma \gamma^{\prime}, z\right)$.

Example 2.1.2. If $\mathcal{R} \subset X \times X$ is a p.m.p. equivalence relation with countable equivalence classes on a standard probability space, then $\mathcal{R}$ is a discrete p.m.p. groupoid with $s(x, y)=y, r(x, y)=x$ and $(x, y)(y, z)=(x, z)$.

Definition 2.1.3. Let $\mathcal{G}$ be a measured groupoid. Then $\mathcal{R}_{\mathcal{G}}$ is the equivalence relation on $X$ given by

$$
x \mathcal{R}_{\mathcal{G}} y \Leftrightarrow \exists \alpha \in \mathcal{G}: s(\alpha)=x, r(\alpha)=y
$$

Definition 2.1.4. A measured groupoid is ergodic if its equivalence relation $\mathcal{R}_{\mathcal{G}}$ is ergodic.

Definition 2.1.5. The full group of $\mathcal{G}$ is defined as $[\mathcal{G}]:=\left[\mathcal{R}_{\mathcal{G}}\right]$. Hence

$$
[\mathcal{G}]=\{\theta \in \operatorname{Aut}(X) \mid \forall x \in X \exists \alpha \in \mathcal{G}: s(\alpha)=x, r(\alpha)=\theta(x)\} .
$$

Definition 2.1.6. Let $\mathcal{G}$ be a discrete p.m.p. groupoid. Then $\operatorname{Aut}(\mathcal{G})$ is the set of Borel maps $\phi: X \rightarrow \mathcal{G}$ such that $s(\phi(x))=x$ for a.e. $x \in X$ and $r \circ \phi$ is a.e. injective.

We will write $\phi$ for $r \circ \phi$. The second condition implies that $\phi$ is a.e. surjective since it is measure preserving. So $\phi$ defines almost everywhere an element of the full group. In fact, every element of the full group is of this form:

Lemma 2.1.7 ([ST10, Lemma 3.10]). For every $\theta \in[\mathcal{G}]$ there is a $\phi \in \operatorname{Aut}(\mathcal{G})$ such that $\underline{\phi}=\theta$ almost everywhere.

For $\phi, \psi \in \operatorname{Aut}(\mathcal{G})$ we define composition and inverse as

$$
\phi \circ \psi(x)=\phi(r(\psi(x))) \psi(x), \quad \phi^{-1}(r(\phi(x)))=(\phi(x))^{-1},
$$

where the inverse is only almost everywhere defined.

Lemma 2.1.8 ([ST10, Lemma 3.2]). For discrete p.m.p. groupoids $\mathcal{G}$ there are countably many $\phi_{n} \in \operatorname{Aut}(\mathcal{G})$ such that $\mathcal{G}=\bigcup \operatorname{im}\left(\phi_{n}\right)$. If $\mathcal{G}$ is ergodic, the union can be made disjoint.

\section{The von Neumann algebra of a discrete p.m.p. groupoid}

Let $\mathcal{G}$ be a discrete p.m.p. groupoid. Similar as for groups, define $\mathbb{C} \mathcal{G}$ to consist of the functions $k \in L^{\infty}\left(\mathcal{G}, \mu_{\mathcal{G}}\right)$ for which there is an $N \in \mathbb{N}$ such that $\left\{\alpha \in \mathcal{G}_{x} \mid k(\alpha) \neq 0\right\}$ and $\left\{\alpha \in \mathcal{G}^{x} \mid k(\alpha) \neq 0\right\}$ contain at most $N$ elements for $\mu$-almost every $x \in X$. Multiplication and involution on $\mathbb{C} \mathcal{G}$ are given by

$$
(k * l)(\gamma)=\sum_{\alpha \beta=\gamma} k(\alpha) l(\beta), \quad k^{*}(\gamma)=\overline{k\left(\gamma^{-1}\right)} .
$$


The left regular representation $\lambda: \mathbb{C} \mathcal{G} \rightarrow \mathcal{B}\left(L^{2}\left(\mathcal{G}, \mu_{\mathcal{G}}\right)\right)$ is given by

$$
\lambda(k)(l):=k * l
$$

for $k, l \in \mathbb{C} \mathcal{G}$ and $\lambda(k)$ extends to a bounded operator on $L^{2}\left(\mathcal{G}, \mu_{\mathcal{G}}\right)$.

The von Neumann algebra of $\mathcal{G}$ is defined as the weak closure of $\lambda(\mathbb{C} \mathcal{G})$,

$$
L \mathcal{G}=\lambda(\mathbb{C G})^{\prime \prime} \subset \mathcal{B}\left(L^{2}\left(\mathcal{G}, \mu_{\mathcal{G}}\right)\right)
$$

$L \mathcal{G}$ is a finite von Neumann algebra with trace $\tau(x)=\left\langle x \chi_{X}, \chi_{X}\right\rangle$, where $\chi_{X}$ is the characteristic function of the units. Then $L^{2}(L \mathcal{G}, \tau)=L^{2}\left(\mathcal{G}, \mu_{\mathcal{G}}\right)$. Hence arbitrary elements of $L \mathcal{G} \subset L^{2}(L \mathcal{G}, \tau)$ are still given by measurable functions $k: \mathcal{G} \rightarrow \mathbb{C}$ for which $\lambda(k)(l)=k * l$ defines a bounded operator on $L^{2}\left(\mathcal{G}, \mu_{\mathcal{G}}\right)$. We will drop $\lambda$ and write $k \in L \mathcal{G}$. Then $\tau(k)=\int_{X} k(x) d \mu$.

Definition 2.1.9. We associate to $\phi \in \operatorname{Aut}(\mathcal{G})$ a unitary element $u_{\phi} \in L \mathcal{G}$ defined by $u_{\phi}=\chi_{\operatorname{im}(\phi)} \in \mathbb{C} \mathcal{G}$.

The $u_{\phi}$ together with $L^{\infty}(X)$ generate $L \mathcal{G}$. We have $u_{\phi} u_{\psi}=u_{\phi \circ \psi}$ and $u_{\phi}^{*}=u_{\phi^{-1}}$.

Lemma 2.1.10. Let $\mathcal{G}$ be a discrete p.m.p. groupoid. Then $L \mathcal{G}$ is a factor if and only if $\mathcal{G}$ is ergodic and almost all the groups $\mathcal{G}_{x}^{x}:=\mathcal{G}_{x} \cap \mathcal{G}^{x}$ are i.c.c..

Proof. If $\mathcal{G}$ is not ergodic, there is an invariant subset $Y \subset X$ of nontrivial measure. Then $\chi_{Y}$ is nontrivial and central in $L \mathcal{G}$, hence $L \mathcal{G}$ is not a factor.

Suppose that $\mathcal{G}_{x}^{x}$ is not i.c.c. for $x \in Y$ a set of positive measure and choose $\phi_{n} \in \operatorname{Aut}(\mathcal{G})$ such that $\mathcal{G} \subset \bigcup \operatorname{im}\left(\phi_{n}\right)$. For every $x \in Y$ let

$$
n_{x}=\min \left\{n \mid \phi_{n}(x) \in \mathcal{G}_{x}^{x} \backslash\{x\}, C l\left(\phi_{n}(x)\right) \text { is finite }\right\},
$$

where $C l(\alpha)$ denotes the conjugacy class of $\alpha$ in $\mathcal{G}_{x}^{x}$. Then for some $N \in \mathbb{N}$, the set

$$
Y^{\prime}=\left\{x \in Y \mid \# C l\left(\phi_{n_{x}}(x)\right)<N\right\}
$$

is of positive measure and $\sum_{x \in Y^{\prime}} \chi_{C l\left(\phi_{n_{x}}(x)\right)} \in \mathbb{C} \mathcal{G}$ is in the center of $L \mathcal{G}$. Hence $L \mathcal{G}$ is not a factor.

If $\mathcal{G}$ is ergodic and almost all $\mathcal{G}_{x}^{x}$ are i.c.c., let $k: \mathcal{G} \rightarrow \mathbb{C}$ be in the center of $L \mathcal{G}$. Then for every $f \in L^{\infty}(X) \subset L \mathcal{G}$

$$
f(r(\alpha)) k(\alpha)=(f k)(\alpha)=(k f)(\alpha)=f(s(\alpha)) k(\alpha)
$$

for almost every $\alpha \in \mathcal{G}$, which implies that $\operatorname{supp}(k) \subset \bigsqcup \mathcal{G}_{x}^{x}$.

Find sections $\phi_{n} \in \operatorname{Aut}\left(\bigsqcup_{x \in X} \mathcal{G}_{x}^{x}\right)$ such that $\bigsqcup_{x \in X} \mathcal{G}_{x}^{x}=\bigcup_{n \in \mathbb{N}} \operatorname{im}\left(\phi_{n}\right)$. Setting $l_{n}=\chi_{\operatorname{im}\left(\phi_{n}\right)}$ gives that for almost every $\alpha$, say for all $\alpha \in \bigsqcup_{x \in Y_{n}} \mathcal{G}_{x}^{x}$ with $\mu\left(Y_{n}\right)=1$, we have 


$$
\begin{aligned}
k\left(\phi_{n}(s(\alpha))^{-1} \alpha\right) & =\sum_{\beta \in \mathcal{G}_{s(\alpha)}} l_{n}(\beta) k\left(\beta^{-1} \alpha\right)=\left(l_{n} k\right)(\alpha)=\left(k l_{n}\right)(\alpha) \\
& =\sum_{\beta \in \mathcal{G}_{s(\alpha)}} k\left(\alpha \beta^{-1}\right) l_{n}(\beta)=k\left(\alpha \phi_{n}(s(\alpha))^{-1}\right) .
\end{aligned}
$$

Define $Y=\bigcap_{n \in \mathbb{N}} Y_{n}$, then $Y$ has measure one and for $x \in Y$ and $\alpha, \alpha^{\prime} \in \mathcal{G}_{x}^{x}$ conjugate we find $k(\alpha)=k\left(\alpha^{\prime}\right)$. Since a.e. $\mathcal{G}_{x}^{x}$ is i.c.c., it follows that $k \in$ $L^{\infty}(X)$. By ergodicity, $k$ is constant. So $L \mathcal{G}$ is a factor.

\section{$2.2 \quad$ Representations and bimodules}

We recall some notions on representations of groupoids, referring to AD05] for more details.

Definition 2.2.1 ([AD05]). If $\mathcal{H}$ is a Hilbert bundle over $\mathcal{G}^{(0)}$ with fibers $\mathcal{H}_{x}$, denote by $\operatorname{Iso}(\mathcal{H})$ the groupoid with elements $(x, V, y)$ for $x, y \in \mathcal{G}^{(0)}$ and $V$ an isomorphism from $\mathcal{H}_{y}$ to $\mathcal{H}_{x}$, and composition given by $(x, V, y)(y, W, z)=$ $(x, V W, z)$.

Definition 2.2.2 ([AD05]). A representation of a Borel groupoid is a Borel homomorphism $U: \mathcal{G} \rightarrow \operatorname{Iso}(\mathcal{H})$ that preserves $\mathcal{G}^{(0)}$, that is,

$$
U(\alpha)=(r(\alpha), \hat{U}(\alpha), s(\alpha))
$$

with $\hat{U}(\alpha): \mathcal{H}_{s(\alpha)} \rightarrow \mathcal{H}_{r(\alpha)}, U(\alpha \beta)=U(\alpha) U(\beta)$ for $(\alpha, \beta) \in \mathcal{G}^{(2)}$ and $U\left(\alpha^{-1}\right)=$ $U(\alpha)^{-1}$. We will identify $U(\alpha)$ and $\hat{U}(\alpha)$.

To connect properties of groupoids and their von Neumann algebras, we need to pass from groupoid representations to bimodules over von Neumann algebras.

\section{From representations to bimodules}

Let $\mathcal{G}$ be a discrete p.m.p. groupoid and let $U: \mathcal{G} \rightarrow \operatorname{Iso}(\mathcal{H})$ be a representation. From this we define $H$ to be the Hilbert bundle over $\mathcal{G}$ with fiber $\mathcal{H}_{r(\alpha)}$ at $\alpha \in \mathcal{G}$. Then $H$ carries the following commuting left and right $L \mathcal{G}$-actions $L$ and $R$ introduced in [Moo82]: Let $\Phi \in H$ and $k \in L \mathcal{G}$, then

$$
\begin{aligned}
\left(L(U)_{k} \Phi\right)(\gamma) & =\sum_{\alpha \circ \beta=\gamma} k(\alpha) U(\alpha) \Phi(\beta) \\
\left(R_{k} \Phi\right)(\gamma) & =\sum_{\alpha \circ \beta=\gamma} \Phi(\alpha) k(\beta) .
\end{aligned}
$$


Definition 2.2.3. The $L \mathcal{G}$-bimodule defined by the construction above is called the associated bimodule for the representation $U: \mathcal{G} \rightarrow \operatorname{Iso}(\mathcal{H})$.

If $\mathcal{G}$ is ergodic, almost every fibre $\mathcal{H}_{x}$ is isomorphic to some Hilbert space $H_{0}$. In this case we have $H=L^{2}\left(\mathcal{G}, H_{0}\right)=L^{2}(\mathcal{G}) \otimes H_{0}$. The following Lemma has a version for general $\mathcal{G}$. We state it in the ergodic case because it makes notation easier.

Lemma 2.2.4. Let $\mathcal{G}$ be an ergodic discrete p.m.p. groupoid. An LG-bimodule $H$ is associated to some representation $U$ of $\mathcal{G}$ if and only if the restriction to a $L^{\infty}(X)$-LG-bimodule is of the form

$$
{ }_{L^{\infty}(X)} H_{L \mathcal{G}}={ }_{\lambda \otimes \mathrm{id}}\left(L^{2}(\mathcal{G}) \otimes H_{0}\right)_{\rho \otimes \mathrm{id}} .
$$

Proof. Given such a bimodule $H$ a corresponding representation can be constructed as follows. Let $\pi$ be the left action of $L \mathcal{G}$ on $H$. Then for $\phi \in \operatorname{Aut}(\mathcal{G})$ the element

$$
f_{\phi}:=(\lambda \otimes \mathrm{id})\left(u_{\phi}^{*}\right) \pi\left(u_{\phi}\right) \in B(H)
$$

belongs to $(\lambda \otimes \mathrm{id})\left(L^{\infty}(X)\right)^{\prime}=L^{\infty}(X) \otimes B\left(H_{0}\right)=L^{\infty}\left(X, B\left(H_{0}\right)\right)$ as for all projections $p_{A} \in L^{\infty}(X)$ onto some $A \subset X$ we have:

$$
\begin{aligned}
(\lambda \otimes \mathrm{id})\left(u_{\phi}^{*}\right) \pi\left(u_{\phi}\right)(\lambda \otimes \mathrm{id})\left(p_{A}\right) & =(\lambda \otimes \mathrm{id})\left(u_{\phi}^{*}\right) \pi\left(u_{\phi} p_{A}\right) \\
& =(\lambda \otimes \mathrm{id})\left(u_{\phi}^{*}\right) \pi\left(p_{\phi(A))} u_{\phi}\right) \\
& =(\lambda \otimes \mathrm{id})\left(u_{\phi}^{*}\right)(\lambda \otimes \mathrm{id})\left(p_{r(\phi(A))}\right) \pi\left(u_{\phi}\right) \\
& =(\lambda \otimes \mathrm{id})\left(u_{\phi}^{*} p_{\phi(A))}\right) \pi\left(u_{\phi}\right) \\
& =(\lambda \otimes \mathrm{id})\left(p_{A}\right)(\lambda \otimes \mathrm{id})\left(u_{\phi}^{*}\right) \pi\left(u_{\phi}\right) .
\end{aligned}
$$

In fact, $f_{\phi} \in L^{\infty}\left(X, U\left(H_{0}\right)\right)$ because $f_{\phi}$ is a product of two unitaries. We assume the $f_{\phi}$ 's to be pointwise defined by picking one version $f_{\phi}: X \rightarrow U\left(H_{0}\right)$. We now would like to define $U: \mathcal{G} \rightarrow U\left(H_{0}\right)$ as $U(\alpha)=f_{\phi}(s(\alpha))$ for some $\phi$ with $\phi(s(\alpha))=\alpha$. To make this well defined, we must first exclude some null sets. To do so, take countably many $\phi_{n} \in \operatorname{Aut}(\mathcal{G})$ such that $\mathcal{G}=\bigcup \operatorname{im}\left(\phi_{n}\right)$. Let $\Gamma$ be the group they generate. Changing the enumeration of the generators we write $\phi_{n}$ also for arbitrary elements of $\Gamma$. Let

$$
X_{n, m}=\left\{x \in X \mid \phi_{n}(x)=\phi_{m}(x)\right\} .
$$

Then $u_{\phi_{n}} p_{X_{n, m}}=u_{\phi_{m}} p_{X_{n, m}} \in L \mathcal{G}$, so for every $\Phi \in L^{2}\left(\mathcal{G}, H_{0}\right)$,

$$
\left(\pi\left(u_{\phi_{n}} p_{X_{n, m}}\right) \Phi\right)(\alpha)=\left(\pi\left(u_{\phi_{m}} p_{X_{n, m}}\right) \Phi\right)(\alpha) \quad \text { for a.e. } \alpha \in \mathcal{G} .
$$

It follows that $f_{\phi_{n}}(x)=f_{\phi_{m}}(x)$ for a.e. $x \in X_{n, m}$ because for $k \in\{n, m\}$ we have

$$
\begin{aligned}
\left(\pi\left(u_{\phi_{k}} p_{X_{n, m}}\right) \Phi\right)(\alpha)=\left((\lambda \otimes \mathrm{id})\left(u_{\phi_{k}}\right) f_{\phi_{k}} \pi\left(p_{X_{n, m}}\right) \Phi\right)(\alpha) \\
=\left(f_{\phi_{k}}(\lambda \otimes \mathrm{id})\left(p_{X_{n, m}}\right) \Phi\right)\left(\phi_{k}^{-1}(r(\alpha)) \alpha\right) \\
\quad= \begin{cases}f_{\phi_{k}}\left(\underline{\phi}_{k}^{-1}(r(\alpha))\right) \Phi\left(\phi_{k}^{-1}(r(\alpha)) \alpha\right) & \text { if } \underline{\phi}_{k}^{-1}(r(\alpha)) \in X_{n, m} \\
0 & \text { else, }\end{cases}
\end{aligned}
$$


so the claim follows by choosing a non-vanishing $\Phi$ and noting that $\left.\left.\left(\underline{\phi}_{n}^{-1}(r(\alpha))\right) \in X_{n, m}\right) \Leftrightarrow\left(\underline{\phi}_{m}^{-1}(r(\alpha))\right) \in X_{n, m}\right) \Rightarrow\left(\phi_{n}{ }^{-1}(r(\alpha))=\phi_{m}{ }^{-1}(r(\alpha))\right)$.

Hence there is a null set $X_{n, m}^{\prime}$ such that $f_{\phi_{n}}(x)=f_{\phi_{m}}(x)$ for all $x \in X_{n, m} \backslash X_{n, m}^{\prime}$, so for $\alpha$ with $s(\alpha) \in X^{\prime}:=X \backslash \bigcup_{n, m \in I} X_{n, m}^{\prime}$ we can define $U(\alpha)=f_{\phi_{n}}(s(\alpha))$ for an $n$ such that $\phi_{n}(s(\alpha))=\alpha$.

Using $f_{\phi} \in L^{\infty}\left(X, B\left(H_{0}\right)\right)$, we find

$$
f_{\phi \circ \psi}=\lambda \otimes \operatorname{id}\left(u_{\psi^{-1}}\right) f_{\phi} \pi\left(u_{\psi}\right)=\left(f_{\phi} \circ \underline{\psi}\right) \cdot f_{\phi} .
$$

Hence we have that $f_{\phi_{n}}\left(\underline{\phi}_{m}(x)\right) f_{\phi_{m}}(x)=f_{\phi_{n} \circ \phi_{m}}(x)$ for a.e $x \in X$, say, for $x \in$ $X^{\prime \prime}$. Let $(\alpha, \beta) \in \mathcal{G}^{(2)}$ with $s(\alpha), s(\beta) \in X^{\prime} \cap X^{\prime \prime}$ and $\phi_{n}(s(\alpha))=\alpha, \phi_{m}(s(\beta))=$ $\beta$. Then $\phi_{n} \circ \phi_{m}(s(\beta))=\alpha \beta$ and

$$
U(\alpha) U(\beta)=f_{\phi_{n}}\left(\underline{\phi}_{m}(s(\beta))\right) f_{\phi_{m}}(s(\beta))=f_{\phi_{n} \circ \phi_{m}}(x)=U(\alpha \beta) .
$$

Hence $U$ is a homomorphism. Now

$$
\begin{aligned}
\left(\pi\left(u_{\phi_{n}}\right) \Phi\right)(\gamma) & =\left((\lambda \otimes \mathrm{id})\left(u_{\phi_{n}}\right) f_{\phi_{n}} \Phi\right)(\gamma)=\sum_{\alpha \beta=\gamma} \chi_{\operatorname{im}\left(\phi_{n}\right)}(\alpha)\left(f_{\phi_{n}} \Phi\right)(\beta) \\
& =\sum_{\alpha \beta=\gamma} \chi_{\operatorname{im}\left(\phi_{n}\right)}(\alpha)\left(f_{\phi_{n}}(r(\beta)) \Phi(\beta)=\sum_{\alpha \beta=\gamma} \chi_{\operatorname{im}\left(\phi_{n}\right)}(\alpha) U(\alpha) \Phi(\beta)\right.
\end{aligned}
$$

as in (2.1). The $u_{\phi_{n}}$ and $L^{\infty}(X)$ generate $L \mathcal{G}$, so since also $\left.\pi\right|_{L^{\infty}(X)}=\lambda \otimes \mathrm{id}$, we find that $\pi$ is the action belonging to $U$ as in (2.1).

\subsection{Almost invariant sections and property $(\mathrm{T})$ for groupoids}

In the definition of property $(\mathrm{T})$ for groupoids (almost) invariant sections will play the same roll as (almost) invariant vectors for groups.

Definition 2.3.1. Let $U: \mathcal{G} \rightarrow \operatorname{Iso}(\mathcal{H})$ be a representation of $\mathcal{G}$. A nonzero section $\xi: \mathcal{G}^{(0)} \rightarrow \mathcal{H}$ is called invariant for $U$ if $U(\alpha) \xi(s(\alpha))=\xi(r(\alpha))$ for almost every $\alpha \in \mathcal{G}$.

There are different versions how to define almost invariant sections. From now on we assume all our spaces to be separable to be able to restrict ourselves to sequences instead of nets.

Definition 2.3.2 ([AD05]). A representation $U: \mathcal{G} \rightarrow \operatorname{Iso}(\mathcal{H})$ has almost invariant unit sections if there is a sequence of Borel sections $\xi_{n}: \mathcal{G}^{(0)} \rightarrow \mathcal{H}$, such that $\left\|\xi_{n}(x)\right\|_{\mathcal{H}_{x}}=1$ for all $n$ and almost every $x \in \mathcal{G}^{(0)}$ and

$$
\left\|U(\alpha) \xi_{n}(s(\alpha))-\xi_{n}(r(\alpha))\right\|_{\mathcal{H}_{r(\alpha)}} \stackrel{n \rightarrow \infty}{\longrightarrow} 0
$$

for almost every $\alpha \in \mathcal{G}$. 
Definition 2.3.3. A representation $U: \mathcal{G} \rightarrow \operatorname{Iso}(\mathcal{H})$ has $L^{2}$-almost invariant sections if there is a sequence of Borel sections $\xi_{n}: \mathcal{G}^{(0)} \rightarrow \mathcal{H}$, such that $\int_{X}\left\|\xi_{n}(x)\right\|^{2} d \mu(x)=1$ and

$$
\left.\int_{X} \| U(\phi(x)) \xi_{n}(x)-\xi_{n}(\underline{\phi}(x))\right) \|_{\mathcal{H}_{\underline{\phi}}(x)}^{2} \mathrm{~d} \mu(x) \stackrel{n \rightarrow \infty}{\longrightarrow} 0
$$

for all $\phi \in \operatorname{Aut}(\mathcal{G})$.

Definition 2.3.4. A representation $U: \mathcal{G} \rightarrow \operatorname{Iso}(\mathcal{H})$ has almost invariant sections if there is a sequence of Borel sections $\xi_{n}: \mathcal{G}^{(0)} \rightarrow \mathcal{H}$, such that $\int_{X}\left\|\xi_{n}(x)\right\|^{2} d \mu(x)=1$, the $\xi_{n}$ do not converge to zero in measure and

$$
\left\|U(\alpha) \xi_{n}(s(\alpha))-\xi_{n}(r(\alpha))\right\|_{\mathcal{H}_{r(\alpha)}} \stackrel{n \rightarrow \infty}{\longrightarrow} 0
$$

for almost every $\alpha \in \mathcal{G}$.

Remark 2.3.5. Definition 2.3.3 is equivalent to the condition that the induced representation of $C^{*}(\mathcal{G}, \mu)$ (see [ADR00, p.141]) weakly contains the trivial representation.

If a representation has almost invariant unit sections, these are also $L^{2}$ almost invariant sections by the Lebesgue convergence theorem. If a representation has $L^{2}$-almost invariant sections, then it also has almost invariant sections by the following lemma. We do not know if the three notions are actually equivalent for ergodic groupoids.

Lemma 2.3.6. Let $\mathcal{G}$ be ergodic. Then if a representation of $\mathcal{G}$ has $L^{2}$-almost invariant sections, it also has almost invariant sections.

Proof. Let $\xi_{n}$ be $L^{2}$-almost invariant sections and let $\phi_{k} \in \operatorname{Aut}(\mathcal{G})$ be such that $\mathcal{G}=\bigcup_{k \in \mathbb{N}} \operatorname{im}\left(\phi_{k}\right)$. Then for every $k \in \mathbb{N}$

$$
\int_{X}\left\|U\left(\phi_{k}(x)\right) \xi_{n}(x)-\xi_{n}\left(\underline{\phi}_{k}(x)\right)\right\|_{\mathcal{H}_{x}}^{2} \rightarrow 0 \quad \text { for } n \rightarrow \infty .
$$

Hence we can pick a subsequence such that $\| U\left(\phi_{k}(x)\right) \xi_{n}(x)-\xi_{n}\left(r\left(\phi_{k}(x)\right) \|_{\mathcal{H}_{x}}\right.$ goes to zero for almost every $x \in X$. A diagonalisation over subsequences makes this true for every $k \in \mathbb{N}$. For this subsequence it is now true that

$$
\left\|U(\alpha) \xi_{n}(s(\alpha))-\xi_{n}(r(\alpha))\right\|_{H_{r(\alpha)}} \rightarrow 0 \text { for a.e. } \alpha \in \mathcal{G} .
$$

Assume for contradiction that the $\xi_{n}$ converge to zero in measure and pick a subsequence such that $\xi_{n} \rightarrow 0$ a.e.. With $\left\|\xi_{n}\right\|_{2}=1$ then there is an $N$ and a set $A \subset X$ such that $\mu(A)<\frac{1}{2}$ and $\left\|\left.\xi_{n}\right|_{A}\right\|_{2}>\frac{3}{4}$ for all $n>N$. By [ST10, Lemma 3.8] there is a $\phi \in \operatorname{Aut}(\mathcal{G})$ such that $\phi^{-1}(A) \subset A^{c}$. Now since the $\xi_{n}$ are $L^{2}$-almost invariant, there is an $n>N$ with

$$
\left\|U(\phi) \xi_{n}-\xi_{n}\right\|_{2}<\frac{1}{4}
$$


where $\left(U(\phi) \xi_{n}\right)(x):=U\left(\phi\left(\underline{\phi}^{-1}(x)\right) \xi_{n}\left(\underline{\phi}^{-1}(x)\right)\right.$. So

$$
\left\|\left.\xi_{n}\right|_{A^{c}}\right\|_{2} \geq\left\|\left.\left(U(\phi) \xi_{n}\right)\right|_{A}\right\|_{2} \geq\left\|\left.\xi_{n}\right|_{A}\right\|_{2}-\left\|\left.\left(U(\phi) \xi_{n}-\xi_{n}\right)\right|_{A}\right\|_{2}>\frac{1}{2}
$$

in contradiction to $\left\|\xi_{n}\right\|_{2}=\left\|\left.\xi_{n}\right|_{A}\right\|_{2}+\left\|\left.\xi_{n}\right|_{A^{c}}\right\|_{2}=1$.

Remark 2.3.7. If $\mathcal{G}$ is ergodic, invariant sections are automatically of constant norm. But it is not true for ergodic $\mathcal{G}$ that almost invariant sections with $\left\|\xi_{n}\right\|_{2}=1$ are approximately unit sections: Let $\mathcal{G}$ be the hyperfinite equivalence relation on $[0,1]$ where two numbers are equivalent if their binary codes coincide from some point on. Then $\xi_{n} \in L^{2}([0,1])$ given by

$$
\xi_{n}(x)= \begin{cases}\sqrt{2} & \text { if } \exists k \in \mathbb{N}: x \in\left[\frac{2 k}{2^{n}}, \frac{2 k+1}{2^{n}}\right) \\ 0 & \text { if } \exists k \in \mathbb{N}: x \in\left[\frac{2 k+1}{2^{n}}, \frac{2 k}{2^{n}}\right)\end{cases}
$$

are almost invariant for the trivial representation of $\mathcal{G}$, but not approximately of constant norm.

However, the constant 1 is an invariant vector for the trivial representation, so the above example does not prove Definition 2.3.2 and Definition 2.3.4 to be different.

As we will see in the proof of the next proposition, the above kind of example cannot happen if $\mathcal{G}$ has property $(\mathrm{T})$.

Definition 2.3.8 ([AD05]). $\mathcal{G}$ has property $(T)$ if every representation $U$ which has almost invariant unit sections already has invariant sections.

The following proposition is certainly known to the experts, but we could not find a reference.

Proposition 2.3.9. We get equivalent characterizations of property (T) when replacing "almost invariant unit section" in Definition 2.3 .8 by "almost invariant sections" or " $L^{2}$-almost invariant sections".

Proof. We show that if $\mathcal{G}$ has property $(\mathrm{T})$ as in Definition 2.3.8 and $\xi_{n}$ are almost invariant sections for a representation $U$, then there is an invariant section for $U$. This proves the claim because, by the discussion before Lemma 2.3.6, having almost invariant unit sections is stronger than having $L^{2}$-almost invariant sections and having almost invariant sections. The proof partly follows [Sch81, 2.3, (1) $\Rightarrow(3)$ ].

Let $f_{n} \in L^{2}(X)$ be given by $f_{n}(x)=\left\|\xi_{n}(x)\right\|$. These functions have 2-norm one and are almost invariant for the representation $V(\alpha) f(s(\alpha))=f(r(\alpha))$ of $\mathcal{G}$ on the Hilbert bundle with fibers $\mathbb{C}$.

Define a sequence of probability measures on $\mathbb{R}$ by

$$
\sigma_{n}(A)=\mu\left(\left\{x \in X \mid f_{n}(x) \in A\right\}\right) .
$$


Then $\left\{\sigma_{n}\right\}$ is uniformly tight, meaning that for every $\varepsilon>0$ there is a compact $K \subset \mathbb{R}$ such that $\sigma_{n}(K) \geq 1-\varepsilon$ for all $n$. This is true because otherwise there would be a subsequence such that $\mu\left(\left\{f_{n}>n\right\}\right) \geq \varepsilon$ and hence $\left\|f_{n}\right\|_{2} \rightarrow \infty$. By [Par67, Theorem II.6.7] it follows that $\overline{\left\{\sigma_{n}\right\}}$ is weak* compact. Let $\sigma$ be a probability measure such that $\sigma_{n} \rightarrow \sigma$ for a subsequence in the weak* topology.

If $\sigma$ is not a Dirac measure, there is an $a \in \mathbb{R}$ such that $\sigma(\{a\})=0$ and $0<\sigma([0, a])<1$. Since $a$ is a continuity point of $\sigma$, we have $\lim \sigma_{n}([0, a])=$ $\sigma([0, a])$. Hence for $C_{n}=\left\{x \in X \mid f_{n}(x)<a\right\}$ we find $\mu\left(C_{n}\right)\left(1-\mu\left(C_{n}\right)\right) \nrightarrow 0$. We claim that $\mu\left(C_{n} \triangle \theta\left(C_{n}\right)\right) \rightarrow 0$ for all $\theta \in[\mathcal{G}]$.

To this end, let $\theta \in[\mathcal{G}]$ and $x \in X$. Then $\theta(x)=r(\alpha)$ for some $\alpha \in \mathcal{G}_{x}$ and

$$
\left|f_{n}(x)-f_{n}(\theta(x))\right| \leq\left\|U(\alpha) \xi_{n}(x)-\xi_{n}(\theta(x))\right\| \rightarrow 0
$$

almost surely. So $\left|f_{n}(x)-f_{n}(\theta(x))\right| \rightarrow 0$ a.e. and hence in measure. Using this and continuity of $\sigma$ at $a$ for every $\varepsilon>0$ and $\theta \in[\mathcal{G}]$ we find a $\delta>0$ and an $N \in \mathbb{N}$ such that for all $n>N$

$\mu\left(\left\{f_{n} \in B_{\delta}(a)\right\}\right)=\sigma_{n}\left(B_{\delta}(a)\right)<\varepsilon$ and $\mu\left(\left\{x \in X|| f_{n}(\theta(x))-f_{n}(x) \mid>\delta\right\}\right)<\varepsilon$.

Then $\mu\left(\theta^{-1}\left(C_{n}\right) \triangle C_{n}\right)<2 \varepsilon$ because otherwise

$$
\begin{aligned}
\mu\left(\left\{x \in X|| f_{n}(\theta(x))-f_{n}(x) \mid>\delta\right\}\right) & \geq \mu\left(\left(\theta^{-1}\left(C_{n}\right) \triangle C_{n}\right) \cap\left\{f_{n} \in B_{\delta}(a)\right\}^{c}\right) \\
& \geq \mu\left(\theta^{-1}\left(C_{n}\right) \triangle C_{n}\right)-\sigma_{n}\left(B_{\delta}(a)\right) \geq \varepsilon .
\end{aligned}
$$

Hence if $\sigma$ is not a Dirac measure, $\mathcal{R}_{\mathcal{G}}$ is not strongly ergodic. Then by [JS87, Theorem 2.2., Remark 2.5] there is an ergodic amenable countable infinite equivalence relation $\mathcal{Q} \subset Y \times Y$ on a probability space $(Y, \nu)$ and a measure preserving map $\Psi: X \rightarrow Y$ mapping $\mathcal{R}_{\mathcal{G}}$ to $\mathcal{Q}$. Since $\mathcal{G}$ has property $(\mathrm{T}), \mathcal{R}_{\mathcal{G}}$ and $\mathcal{Q}$ also have property (T) because every representation of $\mathcal{Q}$ or $\mathcal{R}_{\mathcal{G}}$ with almost invariant unit sections and no invariant section would give a representation of $\mathcal{G}$ with the same properties. But if an amenable countable ergodic equivalence relation has property $(\mathrm{T})$, it is essentially transitive $\mathrm{AD} 05$, Proposition 4.7, Remark 4.6]. This implies that $Y$ is countable and hence finite since $\mathcal{Q}$ is measure preserving, a contradiction.

Thus $\sigma$ is a Dirac measure $\sigma=\delta_{a}$, which means that the $f_{n}$ converge to the constant $a$ in measure. By Definition 2.3.4, the $\xi_{n}$ do not converge to zero in measure, hence $a \neq 0$. This means that the $\left\|\xi_{n}\right\|$ go away from zero in measure and therefore the sections

$$
\xi_{n}^{\prime}(x)= \begin{cases}\xi_{n}(x) /\left\|\xi_{n}(x)\right\| & \text { if } \xi_{n}(x) \neq 0 \\ \xi_{0}(x) & \text { if } \xi_{n}(x)=0\end{cases}
$$

for a fixed section $\xi_{0}$ of constant norm one are still almost invariant and of norm one. So property $(\mathrm{T})$ gives an invariant section. 
Remark 2.3.10. A groupoid is amenable iff the left regular representation has almost invariant unit sections (see [ADR00, Prop. 3.2.14 (v)]). As for property (T) we get an equivalent definition if we use only $L^{2}$-almost invariant sections instead as [ADR00, Prop. 6.1.4 (ii)] shows. We do not know if almost invariant sections as in Definition 2.3.4 also give the same notion of amenability.

\subsection{Almost invariant sections and almost cen- tral vectors}

In this section we compare the notions of having almost central vectors for bimodules and having almost invariant unit sections for representations.

Lemma 2.4.1. Let $U: \mathcal{G} \rightarrow \operatorname{Iso}(\mathcal{H})$ be a representation of an ergodic discrete p.m.p. groupoid such that $\mathcal{H}_{x}=H_{0}$ for almost all $x \in \mathcal{G}^{(0)}$. Let $\xi_{n}: \mathcal{G}^{(0)} \rightarrow H_{0}$ be almost invariant unit sections. Then the functions $\Phi_{n} \in H=L^{2}\left(\mathcal{G}, H_{0}\right)$ defined by

$$
\Phi_{n}(\alpha)= \begin{cases}\xi_{n}(\alpha) & \text { if } \alpha \in \mathcal{G}^{(0)} \\ 0 & \text { else }\end{cases}
$$

are almost central norm one vectors in the associated $L \mathcal{G}$-bimodule.

Proof. For all $k \in L \mathcal{G}$ we have

$$
\left\|L(U)_{k} \Phi_{n}-R_{k} \Phi_{n}\right\|=\left(\int_{\mathcal{G}}|k(\alpha)|^{2}\left\|U(\alpha) \xi_{n}(s(\alpha))-\xi_{n}(r(\alpha))\right\|^{2} d \mu_{\mathcal{G}}(\alpha)\right)^{\frac{1}{2}} .
$$

Let $k \in \mathbb{C G}$ with $k<C$ a.e. and such that $K_{x}:=\left\{\alpha \in \mathcal{G}_{x} \mid k(\alpha) \neq 0\right\}$ has at most $N$ elements for a.e. $x \in X$. Then

$$
\left\|L(U)_{k} \Phi_{n}-R_{k} \Phi_{n}\right\| \leq C\left(N \int_{X} \max _{\alpha \in K_{x}}\left\{\left\|U(\alpha) \xi_{n}(x)-\xi_{n}(r(\alpha))\right\|^{2}\right\} d \mu(x)\right)^{\frac{1}{2}} .
$$

This converges to zero because $x \mapsto \max _{\alpha \in K_{x}}\left\{\left\|U\left(\alpha_{x}\right) \xi_{n}(x)-\xi_{n}\left(r\left(\alpha_{x}\right)\right)\right\|^{2}\right\}$ converges pointwise and therefore in 2-norm as it is bounded by 4 . Now the statement for general $k \in L \mathcal{G}$ follows because by the Kaplansky density theorem we can strongly approximate $k$ by elements of $\mathbb{C G}$ that are uniformly bounded in operator norm.

Lemma 2.4.2. Let $\mathcal{G}$ be discrete p.m.p. groupoid such that $L \mathcal{G}$ is a factor and let $U: \mathcal{G} \rightarrow \operatorname{Iso}(\mathcal{H})$ be a representation. Let $H=L\left(\mathcal{G}, H_{0}\right)$ be the associated $L \mathcal{G}$-bimodule. Then if $\Phi \in H$ is central for the actions of $L \mathcal{G}$, it is supported on $\mathcal{G}^{(0)}$ and $\xi: \mathcal{G}^{(0)} \rightarrow H_{0}$ defined as $\xi(x)=\Phi(x)$ is an invariant unit section for $U$. 
Proof. For every $f \in L^{\infty}(X)$ we have

$$
f(r(\alpha)) \Phi(\alpha)=\left(L(U)_{f} \Phi\right)(\alpha)=\left(R_{f} \Phi\right)(\alpha)=f(s(\alpha)) \Phi(\alpha)
$$

almost everywhere, which implies that $\operatorname{supp}(\Phi) \subset \bigsqcup \mathcal{G}_{x}^{x}$. Now for general $k \in L \mathcal{G}$ we have for almost every $\alpha \in \bigsqcup \mathcal{G}_{x}^{x}$

$$
\sum_{\beta \in \mathcal{G}_{x}} k(\beta) U(\beta) \Phi\left(\beta^{-1} \circ \alpha\right)=\left(L(U)_{k} \Phi\right)(\alpha)=\left(R_{k} \Phi\right)(\alpha)=\sum_{\beta \in \mathcal{G}_{x}} \Phi\left(\alpha \circ \beta^{-1}\right) k(\beta) .
$$

Find sections $\phi_{n} \in \operatorname{Aut}\left(\bigsqcup_{x \in X} \mathcal{G}_{x}^{x}\right)$ such that $\bigsqcup_{x \in X} \mathcal{G}_{x}^{x}=\bigcup_{n \in \mathbb{N}} \operatorname{im}\left(\phi_{n}\right)$. Setting $k=\chi_{\mathrm{im}\left(\phi_{n}\right)}$ gives

$$
U\left(\phi_{n}(x)\right) \Phi\left(\phi_{n}(x)^{-1} \alpha\right)=\Phi\left(\alpha \phi_{n}(x)^{-1}\right)
$$

for almost every $\alpha$, say, for all $\alpha \in \bigsqcup_{x \in Y_{n}} \mathcal{G}_{x}^{x}$ with $\mu\left(Y_{n}\right)=1$. Then $Y=$ $\bigcap_{n \in \mathbb{N}} Y_{n}$ has measure one, and it follows from above that for $x \in Y$

$$
\alpha, \alpha^{\prime} \in \mathcal{G}_{x}^{x} \text { conjugate } \Rightarrow\|\Phi(\alpha)\|=\left\|\Phi\left(\alpha^{\prime}\right)\right\| .
$$

Since $L \mathcal{G}$ is a factor, almost all the $\mathcal{G}_{x}^{x}$ are i.c.c.. So we find that $\operatorname{supp}(\Phi) \subset \mathcal{G}^{(0)}$ because $\Phi \in L^{2}\left(\mathcal{G}, H_{0}\right)$.

Now $\xi$ is an invariant section for $U$ because if $\phi \in \operatorname{Aut}(\mathcal{G})$ and $k=\chi_{\operatorname{im}(\phi)}$, we have

$$
k(\alpha) U(\alpha) \xi(s(\alpha))=L_{k}(U) \xi(\alpha)=R_{k} \xi(\alpha)=k(\alpha) \xi(r(\alpha)),
$$

so $U(\alpha) \xi(s(\alpha))=\xi(r(\alpha))$ for almost all $\alpha \in \operatorname{im}(\phi)$. The claim follows because there are $\phi_{n} \in \operatorname{Aut}(\mathcal{G})$ such that $\mathcal{G}=\bigcup_{n \in \mathbb{N}} \operatorname{im}\left(\phi_{n}\right)$.

\subsection{Corigidity and rigidity}

There are different definitions for a von Neumann algebra $M$ to have property (T) relative to a von Neumann algebra $N \subset M$ :

Definition 2.5.1. Let $M$ be a $\mathrm{II}_{1}$ factor and $N \subset M$ a von Neumann subalgebra.

i) $N \subset M$ is called corigid if every $M$-bimodule $H$ with $N$-central norm one vectors $\xi_{n} \in H$ such that $\left\|x \xi_{n}-\xi_{n} x\right\| \rightarrow 0$ for all $x \in M$ contains a non-zero $M$-central vector.

ii) $N \subset M$ has Anantharaman-Delaroche's property $T$ if every $M$-bimodule $H$ such that

$$
{ }_{N} H_{M}={ }_{\lambda \otimes \mathrm{id}}\left(L^{2}(M) \otimes H_{0}\right)_{\rho \otimes \mathrm{id}}
$$

that has norm one $N$-central vectors $\xi_{n} \in H$ such that

$$
\left\|x \xi_{n}-\xi_{n} x\right\| \rightarrow 0 \quad \text { for all } x \in M,
$$

contains a non-zero $M$-central vector $\xi_{0}$. 
iii) $N \subset M$ has Moore's property $T$ if every $M$-bimodule $H$ such that

$$
{ }_{N} H_{M}={ }_{\lambda \otimes \mathrm{id}}\left(L^{2}(M) \otimes H_{0}\right)_{\rho \otimes \mathrm{id}}
$$

that has norm one vectors $\xi_{n} \in H$ such that $\left\|x \xi_{n}-\xi_{n} x\right\| \rightarrow 0$ for all $x \in M$ contains a non-zero $M$-central vector.

Remark 2.5.2. The notion of corigidity originates from [Pop86], the adjective "corigid" first appears in Pop06 (corigid inclusions were called "rigid" in [Pop86, and now rigidity is a different notion, see Definition 2.5.3. In [AD87] corigidity is called property $T^{\prime}$ for $(M, N)$. The last two definitions come from [AD87] and [Moo82] respectively.

It is clear that Anantharaman-Delaroche's property $(\mathrm{T})$ is weaker than both Moore's property (T) and corigidity.

Definition 2.5.3. An inclusion of finite von Neumann algebras $N \subset M$ is called rigid if for every $\varepsilon>0$ there is a finite set $F \subset M$ and a $\delta>0$, such that for every $M$-bimodule $H$ with a vector $\xi \in H$ satisfying $\|\langle x \xi, \xi\rangle-\tau(x)\|<\delta\|x\|$ and $\|\langle\xi x, \xi\rangle-\tau(x)\|<\delta\|x\|$ for all $x \in M$ and $\|x \xi-\xi x\|<\delta$ for all $x \in F$ there is a an $N$-central vector $\xi_{0} \in H$ that satisfies $\left\|\xi-\xi_{0}\right\|<\varepsilon$.

Definition 2.5.3 is independent of the chosen trace $\tau$.

Definition 2.5.4. $M$ has property (T) if $M \subset M$ is rigid.

$L \Gamma$ has property $(\mathrm{T})$ iff $\Gamma$ has property $(\mathrm{T})$, hence this is actually a generalization.

Proposition 2.5.5 ([Pop06, Proposition 5.9]). Let $M$ be a $I I_{1}$ factor and $N \subset M$ a von Neumann subalgebra. Then $M$ has property $(T)$ if and only if $N \subset M$ is both rigid and corigid.

\subsection{Strong extensions}

The theory of strong extension of groupoids is developed in [ST10]. We recall the relevant information.

For subgroupoids $\mathcal{S} \subset \mathcal{G}$ we always assume $\mathcal{S}^{(0)}=\mathcal{G}^{(0)}=X$.

Definition 2.6.1. Let $\mathcal{S} \subset \mathcal{G}$ be a subgroupoid of a discrete measured groupoid. Then $\operatorname{Aut}_{\mathcal{S}}(\mathcal{G})$ is the set of all $\phi \in \operatorname{Aut}(\mathcal{G})$ such that

$$
\alpha \in \mathcal{S} \Leftrightarrow \phi(r(\alpha)) \alpha \phi(s(\alpha))^{-1} \in \mathcal{S}
$$

for every $\alpha \in \mathcal{G}$. 
Definition 2.6.2. A subgroupoid $\mathcal{S} \subset \mathcal{G}$ is strongly normal if there is a countable family $\left\{\phi_{n}\right\}_{n \in I} \subset \operatorname{Aut}_{\mathcal{S}}(\mathcal{G})$ such that for a.e. $\alpha \in \mathcal{G}$ there exists exactly one $n \in I$ such that $\phi_{n}(r(\alpha)) \alpha \in \mathcal{S}$.

Definition 2.6.3. Let $q: \mathcal{G} \rightarrow \mathcal{Q}$ be a homomorphism of discrete measured groupoids with unit spaces $(X, \mu)$ and $(Y, \nu)$ such that $\nu=q_{*} \mu$. Let

$$
\mu=\int_{Y} \nu_{y} d \nu(y)
$$

be the measure disintegration with respect to $q:(X, \mu) \rightarrow(Y, \nu)$ and let $\mathcal{S}=$ $q^{-1}(Y)$. Then

i) $q$ is called a surjection if for a.e. $x \in X$ and a.e. $\alpha \in \mathcal{Q}$ with $s(\alpha)=q(x)$ there exists a $\beta \in \mathcal{G}$ such that $q(\beta)=\alpha$.

ii) $q$ is called a strong surjection if it is a surjection and $\left(\left.\mathcal{S}\right|_{q^{-1}(y)}, \nu_{y}\right)$ is ergodic for a.e. $y \in Y$.

Theorem 2.6.4 ([ST10, Theorem 3.12]). Let $\mathcal{S}$ be a strongly normal subgroupoid of an ergodic p.m.p. groupoid. Then there is a strong surjection $q: \mathcal{G} \rightarrow \mathcal{Q}$ onto an ergodic discrete measured groupoid, called the quotient of $\mathcal{G}$ by $\mathcal{S}$ such that

i) $\operatorname{ker}(q):=q^{-1}\left(\mathcal{Q}^{(0)}\right)=\mathcal{S}$,

ii) for any ergodic measured groupoid $\mathcal{Q}^{\prime}$ and any homomorphism $q^{\prime}: \mathcal{G} \rightarrow \mathcal{Q}^{\prime}$ with $\mathcal{S} \subset \operatorname{ker}\left(q^{\prime}\right)$ there is a measure preserving homomorphism $\kappa: \mathcal{Q} \rightarrow$ $\mathcal{Q}^{\prime}$ such that $\kappa \circ q=q^{\prime}$.

In such a situation we write $1 \rightarrow \mathcal{S} \rightarrow \mathcal{G} \stackrel{q}{\rightarrow} \mathcal{Q} \rightarrow 1$.

Theorem 2.6.5 ([ST10, Theorem 3.11]). Let $q: \mathcal{G} \rightarrow \mathcal{Q}$ be a strong surjection of ergodic, discrete p.m.p. groupoids. Then the kernel is a strongly normal subgroupoid.

The following lemma will be useful later.

Lemma 2.6.6. Let $1 \rightarrow \mathcal{S} \rightarrow \mathcal{G} \stackrel{q}{\rightarrow} \mathcal{Q} \rightarrow 1$ be a strong extensions of groupoids with $(X, \mu)$ and $(Y, \nu)$ as in Definition 2.6.3. Then the relative commutant $L^{\infty}(X) \cap L \mathcal{S}^{\prime}$ is isomorphic to $L^{\infty}(Y)$. More precisely, $L^{\infty}(X) \cap L \mathcal{S}^{\prime}=L^{\infty}(Y) \circ$ $q$.

Proof. Let $f \in L^{\infty}(X) \cap L \mathcal{S}^{\prime}$. Then

$$
u_{\phi} f=f u_{\phi} \quad \forall \phi \in \operatorname{Aut}(\mathcal{S}) .
$$

Since $f u_{\phi}=u_{\phi}(f \circ \phi)$, these are exactly those $f$ for which $f \circ \phi=f$ a.e. $\forall \phi \in \operatorname{Aut}(\mathcal{S})$, i.e., $f$ is $[\mathcal{S}]$-invariant. As $q$ is a strong surjection, $\left(\left.\overline{\mathcal{S}}\right|_{q^{-1}(y)}, \nu_{y}\right)$ 
is ergodic for almost every $y \in Y$. So $f$ is essentially constant on almost all $\left(q^{-1}(y), \nu_{y}\right)$ and therefore lies in $L^{\infty}(Y) \circ q$.

If $f \in L^{\infty}(Y) \circ q$, it follows that $u_{\phi} f=f u_{\phi}$ for all $\phi \in \operatorname{Aut}(\mathcal{S})$. Since $f$ clearly commutes with $L^{\infty}(X)$, which together with these $u_{\phi}$ generates $L \mathcal{S}$, we get $f \in L^{\infty}(X) \cap L \mathcal{S}^{\prime}$.

\subsection{Property ( $\mathrm{T}$ ) for groupoids and corigidity}

In this section we prove the main result of this chapter connecting property ( $\mathrm{T}$ ) of a quotient of groupoids to coriditiy of the inclusion of the corresponding von Neumann algebras. For groups this is the well known fact that $\Gamma / \Lambda$ has property (T) iff the inclusion $L \Lambda \subset L \Gamma$ is corigid. For ergodic equivalence relations it comes down to the result that $\mathcal{R}$ has property (T) iff $L^{\infty}(X) \subset L \mathcal{R}$ is corigid.

Theorem 2.7.1. Let

$$
1 \rightarrow \mathcal{S} \rightarrow \mathcal{G} \stackrel{q}{\rightarrow} \mathcal{Q} \rightarrow 1
$$

be a strong extension of discrete p.m.p. groupoids such that $L \mathcal{G}$ is a factor. Then $\mathcal{Q}$ has property $(T)$ if and only if $L \mathcal{S} \subset L \mathcal{G}$ is corigid. It is also equivalent to $L \mathcal{S} \subset L \mathcal{G}$ having Anantharaman-Delaroche's $(T)$.

We will cite some lemmas which we prove afterwards without using Theorem 2.7.1.

Proof. Since Anantharaman-Delaroche's (T) is clearly weaker than corigidity, we only have to show that $\mathcal{Q}$ has property (T) if $L \mathcal{S} \subset L \mathcal{G}$ has AnantharamanDelaroche's (T) and that $L \mathcal{S} \subset L \mathcal{G}$ is corigid if $\mathcal{Q}$ has property (T). By Proposition 2.3.9 we may assume almost invariant unit sections in order to prove property $(\mathrm{T})$.

We write $Y$ for $\mathcal{Q}^{(0)}$. Suppose $L \mathcal{S} \subset L \mathcal{G}$ has Anantharaman-Delaroche's property $(\mathrm{T})$ and let $U: \mathcal{Q} \rightarrow \operatorname{Iso}(\mathcal{H})$ be a representation of $\mathcal{Q}$ with almost invariant sections. Since $\mathcal{Q}$ is ergodic, $\mathcal{H}_{y}=H_{0}$ for a.e. $y \in Y$ and some Hilbert space $H_{0}$. Consider the corresponding representation of $\mathcal{G}$ given by $U^{\prime}=U \circ q$ on the Hilbert space $\int_{X} \mathcal{H}_{x} d \mu$, where $\mathcal{H}_{x}=H_{0}$ for all $x \in X$. Let $H=L^{2}\left(\mathcal{G}, H_{0}\right)$ be the $L \mathcal{G}$-bimodule constructed out of $U^{\prime}$ as in 2.2 .

If $U$ has almost invariant unit sections $\xi_{n}: \mathcal{Q}^{(0)} \rightarrow H_{0}$, the sections $\xi_{n}^{\prime}:=$ $\xi_{n} \circ q$ are almost invariant unit sections for $U^{\prime}$ because $q$ is measure preserving. By Lemma 2.4.1, we get that the vectors

$$
\Phi_{n}(\alpha)= \begin{cases}\xi_{n}^{\prime}(\alpha) & \text { if } \alpha \in \mathcal{G}^{(0)} \\ 0 & \text { else }\end{cases}
$$

are almost central in $H$. They are central for $L \mathcal{S}$ by Lemma 2.7.2. 
Hence by Anantharaman-Delaroche's property $(\mathrm{T})$ there is a norm one vector $\Phi \in L^{2}\left(\mathcal{G}, H_{0}\right)$ that is central for the whole of $L \mathcal{G}$. By Lemma 2.4.2 it follows that $\xi^{\prime}(x)=\Phi(x)$ is an invariant unit section for $U^{\prime}$.

By Theorem 2.6.4. $\left(\mathcal{S}_{q^{-1}(y)}, \nu_{y}\right)$ is ergodic for almost all $y \in \mathcal{Q}^{(0)}$, and for those $y,\left.\xi^{\prime}\right|_{q^{-1}(y)}$ is essentially constant since it is invariant under $\mathcal{S}$. So there is a well-defined section $\xi: \mathcal{Q}^{(0)} \rightarrow \mathcal{H}$ given by $\xi \circ q=\xi^{\prime}$. It has norm one because $q$ is measure preserving, and it is $U$-invariant by construction. Hence $\mathcal{Q}$ has property $(\mathrm{T})$.

For the other direction, let $\mathcal{Q}$ have property $(\mathrm{T})$ and let $H$ be an $L \mathcal{G}$ bimodule with almost central vectors that are central for $L \mathcal{S}$.

Let $H^{\prime} \subset H$ be the subspace of vectors in $H$ that are central for $L \mathcal{S}$. $H^{\prime}$ is a Hilbert space since the action is by continuous operators. It is invariant under the conjugation action $\xi \mapsto u_{\phi} \xi u_{\phi}^{*}$ of $\operatorname{Aut}_{\mathcal{S}}(\mathcal{G})$ on $H$. To prove this we check that $u_{\phi} \xi u_{\phi}^{*}$ with $\phi \in \operatorname{Aut}_{\mathcal{S}}(\mathcal{G})$ and $\xi \in H^{\prime}$ commutes with $u_{\psi}$ for $\psi \in \operatorname{Aut}(\mathcal{S})$ and with $L^{\infty}(X)$, which together generate $L \mathcal{S}$. By the definition of $\operatorname{Aut}_{\mathcal{S}}(\mathcal{G})$ we have $u_{\phi}^{*} u_{\psi} u_{\phi} \in L \mathcal{S}$, which implies that

$$
u_{\psi} u_{\phi} \xi u_{\phi}^{*} u_{\psi}^{*}=u_{\phi}\left(u_{\phi}^{*} u_{\psi} u_{\phi}\right) \xi\left(u_{\phi}^{*} u_{\psi} u_{\phi}\right)^{*} u_{\phi}^{*}=u_{\phi} \xi u_{\phi}^{*} .
$$

Using $L^{\infty}(X) \subset L \mathcal{S}$ we get for all $f \in L^{\infty}(X)$

$$
f u_{\phi} \xi u_{\phi}^{*}=u_{\phi}(f \circ \underline{\phi}) \xi u_{\phi}^{*}=u_{\phi} \xi(f \circ \underline{\phi}) u_{\phi}^{*}=u_{\phi} \xi u_{\phi}^{*} f .
$$

$H^{\prime}$ is also invariant under left multiplication of $L^{\infty}(X) \cap L \mathcal{S}^{\prime}$ because for $\xi \in H^{\prime}, f \in L^{\infty}(X) \cap L \mathcal{S}^{\prime}, \psi \in \operatorname{Aut}(\mathcal{S})$ and $g \in L^{\infty}(X)$ we find $f \xi=f u_{\psi} \xi u_{\psi}^{*}=$ $u_{\psi} f \xi u_{\psi}^{*}$ and $g f \xi=f \xi g$.

By Lemma 2.6.6 we have $L^{\infty}(X) \cap L \mathcal{S}^{\prime} \cong L^{\infty}(Y)$. So Dix81, Theorem II.6.2.2] gives a decomposition

$$
H^{\prime}=\int_{Y} H_{y} d \nu(y)
$$

such that the action of $L^{\infty}(Y)$ is by pointwise multiplication.

For $\phi \in \operatorname{Aut}_{\mathcal{S}}(\mathcal{G}), \phi: X \rightarrow X$ is also well-defined on the quotient, $\phi: Y \rightarrow Y$ because $q \circ \phi: X \rightarrow \bar{Y}$ is $[\mathcal{S}]$-invariant by Definition 2.6.1 and for a.e. $y \in Y$ $\left(\left.\mathcal{S}\right|_{q^{-1}(y)}, \nu_{y}\right)$ is ergodic, so $q \circ \underline{\phi}$ is essentially constant on $\left(q^{-1}(y), \nu_{y}\right)$.

Let now $\phi \in \operatorname{Aut}_{\mathcal{S}}(\mathcal{G})$ and $c\left(u_{\phi}\right): H^{\prime} \rightarrow H^{\prime}$ be conjugation with $u_{\phi} \in$ $U(L \mathcal{G})$. For every $A \subset Y$ we have for the corresponding projection $p_{A} \in L^{\infty}(Y)$

$$
c\left(u_{\phi}\right) p_{A} \xi=u_{\phi} p_{A} \xi u_{\phi}^{*}=p_{\underline{\phi}(A)} u_{\phi} \xi u_{\phi}^{*}=p_{\underline{\phi}(A)} c\left(u_{\phi}\right) \xi .
$$

So by [Dix81, Theorem II.2.5.1], $c\left(u_{\phi}\right)$ is a diagonal operator from $\int H_{y} d \nu(y)$ to $\int H_{\underline{\phi}(y)} d \nu(y)$ :

$$
c\left(u_{\phi}\right)=\int_{Y} c\left(u_{\phi}\right)_{y} d \nu(y), \text { for some } c\left(u_{\phi}\right)_{y}: H_{y} \rightarrow H_{\underline{\phi}(y)} .
$$


Let $\phi_{n} \in \operatorname{Aut}_{\mathcal{S}} \mathcal{G}$ be the sections from Definition 2.6.2. Then for every $\alpha \in \mathcal{G}$ there is exactly one $n$ such that $\phi_{n}(s(\alpha)) \in \mathcal{S} \alpha$. Define $U^{\prime}(\alpha): H_{q(x)} \rightarrow H_{q(y)}$ for $\alpha \in \mathcal{G}_{x}^{y}$ as

$$
U^{\prime}(\alpha)=c\left(u_{\phi_{n}}\right)_{q(x)} \text { for the } \phi_{n} \text { with } \phi_{n}(s(\alpha)) \in \mathcal{S} \alpha .
$$

This is a groupoid homomorphism with $\mathcal{S} \subset \operatorname{ker}\left(U^{\prime}\right)$ a.e. by Lemma 2.7.3. which implies that $U^{\prime}=U \circ q$ a.e. for a representation $U$ of $Q$ on $H^{\prime}$ by Theorem 2.6.4.

Now let $\xi_{i} \in H$ be almost central norm one vectors that are central for the action of $L \mathcal{S}$. Hence $\xi_{i} \in H^{\prime}$. Then the $\xi_{i}$ are $L^{2}$-almost invariant for $U$ by Lemma 2.7.4. So by Lemma 2.3.6 $U$ has almost invariant sections and property (T) of $\mathcal{Q}$ gives a non-zero $\xi \in H^{\prime}$ such that $U(\alpha) \xi(s(\alpha))=\xi(r(\alpha))$ for a.e. $\alpha \in \mathcal{Q}$. Since $U^{\prime}=U \circ q$, we get $U^{\prime}(\alpha) \xi(q(s(\alpha)))=\xi(q(r(\alpha)))$ for a.e. $\alpha \in \mathcal{G}$ and hence $u_{\phi_{n}} \xi u_{\phi_{n}}^{*}=\xi$ for every $n \in \mathbb{N}$. These $u_{\phi_{n}}$ generate $L \mathcal{G}$ together with $L \mathcal{S}$, so since $\xi$ is also central for $L \mathcal{S}, x \xi=\xi x$ for all $x \in L \mathcal{G}$.

Lemma 2.7.2. Let $1 \rightarrow \mathcal{S} \rightarrow \mathcal{G} \rightarrow \mathcal{Q} \rightarrow 1$ be a strong extension of discrete p.m.p. groupoids. Let $U$ be a representation of $\mathcal{Q}$ and $U^{\prime}=U \circ q$. Let $H$ be the $L \mathcal{G}$-bimodule corresponding to $U^{\prime}$ as constructed in 2.2 and let $\Phi \in H$ satisfy $\operatorname{supp}(\Phi) \subset \mathcal{G}^{(0)}$. Then $\Phi$ is central for $L \mathcal{S}$.

Proof. The vector $\Phi$ is central for $L^{\infty}(X)$ since $\operatorname{supp}(\Phi) \subset X$. Let $\psi \in \operatorname{Aut}(\mathcal{S})$. Then

$$
u_{\psi} \Phi u_{\psi}^{*}(\alpha)=\sum_{\beta \in \mathcal{G}^{r(\alpha)}} \chi_{\operatorname{im}(\psi)}(\beta) U^{\prime}(\beta) \Phi(s(\beta)) \chi_{\operatorname{im}\left(\psi^{-1}\right)}\left(\beta^{-1} \alpha\right)
$$

The first factor is non-zero iff $\beta=\psi\left(\underline{\psi}^{-1}(s(\alpha))\right)$ and the last one iff $\psi^{-1}(s(\alpha))=$ $\beta^{-1} \alpha$. So $u_{\psi} \Phi u_{\psi}^{*}(\alpha)$ is non-zero only if

$$
\alpha=\beta \psi^{-1}(s(\alpha))=\psi\left(\underline{\psi}^{-1}(s(\alpha))\right) \psi^{-1}(s(\alpha))=s(\alpha) .
$$

Thus $u_{\psi} \Phi u_{\psi}^{*}$ is again supported on $X$. For $x \in X$ the above formula gives

$$
u_{\psi} \Phi u_{\psi}^{*}(x)=(U \circ q)\left(\psi\left(\underline{\psi}^{-1}(x)\right)\right) \Phi(x)=U(x) \Phi(x)=\Phi(x) .
$$

Lemma 2.7.3. The map $U^{\prime}: \mathcal{G} \rightarrow \operatorname{Iso}\left(H^{\prime}\right)$ defined in $(2.4)$ is a groupoid homomorphism and $\mathcal{S} \subset \operatorname{ker}\left(U^{\prime}\right)$ a.e..

Proof. For every $\phi \in \operatorname{Aut}(\mathcal{G})$ let

$$
\mathcal{G}_{\phi}=\{\alpha \in \mathcal{G} \mid \phi(s(\alpha)) \in \mathcal{S} \alpha\} .
$$

Then $\mathcal{G}=\bigsqcup \mathcal{G}_{\phi_{n}}$ and $\mathcal{G}_{\phi_{n}}=\mathcal{G}_{\phi_{n}} \mathcal{S}$ because if $\alpha \in \mathcal{G}_{\phi_{n}}$ and $\sigma \in \mathcal{S}$ are composable, we get, using $\phi_{n} \in \operatorname{Aut}_{\mathcal{S}}(\mathcal{G})$,

$$
\phi_{n}(s(\alpha \sigma))(\alpha \sigma)^{-1}=\phi_{n}(s(\sigma)) \sigma^{-1} \phi_{n}(s(\alpha))^{-1} \phi_{n}(s(\alpha)) \alpha^{-1} \in \mathcal{S} .
$$


Let now

$$
\mathcal{G}_{n, m, k}=\mathcal{G}_{\phi_{n}} \mathcal{G}_{\phi_{m}} \cap \mathcal{G}_{\phi_{k}} .
$$

Then $\mathcal{G}=\bigcup_{n, m, k \in \mathbb{N}} \mathcal{G}_{n, m, k}$. If $\gamma=\alpha \beta \in \mathcal{G}_{\phi_{\gamma}}$ with $\alpha \in \mathcal{G}_{\phi_{\alpha}}$ and $\beta \in \mathcal{G}_{\phi_{\beta}}$, $\phi_{\alpha}, \phi_{\beta}, \phi_{\gamma} \in\left\{\phi_{n}\right\}_{n \in I}$, we find

$$
\phi_{\gamma}(s(\beta)) \beta^{-1} \alpha^{-1}, \phi_{\alpha}(s(\alpha)) \alpha^{-1} \in \mathcal{S},
$$

so $\phi_{\gamma}(s(\beta)) \beta^{-1} \phi_{\alpha}(s(\alpha))^{-1} \in \mathcal{S}$. Conjugation with $\phi_{\alpha}^{-1} \in \operatorname{Aut}_{\mathcal{S}}(\mathcal{G})$ gives

$$
\left(\phi_{\alpha}^{-1} \circ \phi_{\gamma}\right)(s(\beta)) \beta^{-1} \in \mathcal{S} \text {. }
$$

Together with $\phi_{\beta}(s(\beta)) \beta^{-1} \in \mathcal{S}$ this implies $\left(\phi_{\alpha}^{-1} \circ \phi_{\gamma}\right)(s(\beta)) \phi_{\beta}(s(\beta))^{-1} \in \mathcal{S}$ or, by conjugation with $\phi_{\beta}^{-1} \in \operatorname{Aut}_{\mathcal{S}}(\mathcal{G})$,

$$
\left.\left(\phi_{\beta}^{-1} \circ \phi_{\alpha}^{-1} \circ \phi_{\gamma}\right)(s(\beta))\right) \in \mathcal{S} \text {. }
$$

So we find that

$$
\left.\left.\phi_{m}^{-1} \circ \phi_{n}^{-1} \circ \phi_{k}\right|_{s\left(\mathcal{G}_{n, m, k}\right)} \in \operatorname{Aut}(\mathcal{S})\right|_{s\left(\mathcal{G}_{n, m, k}\right)} .
$$

Extend it to a section $\psi \in \operatorname{Aut}(\mathcal{S})$ (This is possible for example by Claim in the proof of [ST10, Lem. 3.2]). Then

$$
u_{\phi_{k}} p_{s\left(\mathcal{G}_{n, m, k}\right)}=u_{\phi_{n} \circ \phi_{m} \circ \psi} p_{s\left(\mathcal{G}_{n, m, k}\right)},
$$

so using that $H^{\prime}$ is central for $L^{\infty}(X)$ and that $c\left(u_{\psi}\right)=\operatorname{id}_{H^{\prime}}$ we get

$$
c\left(u_{\phi_{k}}\right) p_{s\left(\mathcal{G}_{n, m, k}\right)}=c\left(u_{\phi_{k}} p_{s\left(\mathcal{G}_{n, m, k}\right)}\right)=c\left(u_{\phi_{n} \circ \phi_{m} \circ \psi} p_{s\left(\mathcal{G}_{n, m, k}\right)}\right)=c\left(u_{\phi_{n} \circ \phi_{m}}\right) p_{s\left(\mathcal{G}_{n, m, k}\right)} \text {. }
$$

Since $s\left(\mathcal{G}_{n, m, k}\right)$ is, by $(2.5)$, an $[\mathcal{S}]$-invariant subset of $X$, we can also regard it as a subset of $Y=\mathcal{Q}^{(0)}$ and get $c\left(u_{\phi_{n} \circ \phi_{m}}\right)_{y}=c\left(u_{\phi_{k}}\right)_{y}$ for almost every $y \in s\left(\mathcal{G}_{n, m, k}\right)$, say for all $y \in s\left(\mathcal{G}_{n, m, k}\right) \backslash Y_{n, m, k}$ with $Y_{n, m, k}$ of measure zero. If $\mu\left(s\left(\mathcal{G}_{n, m, k}\right)\right)=0$, we choose $Y_{n, m, k}=s\left(\mathcal{G}_{n, m, k}\right)$. Moreover, define

$$
Z_{n, m}=\left\{y \in Y \mid c\left(u_{\phi_{n} \circ \phi_{m}}\right)_{y} \neq c\left(u_{\phi_{n}}\right)_{\underline{\phi}_{m}(y)} c\left(u_{\phi_{m}}\right)_{y}\right\} .
$$

Let now

$$
Y^{\prime}=Y \backslash\left(\bigcup_{n, m, k \in \mathbb{N}} Y_{n, m, k} \cup Z_{n, m}\right) .
$$

Then $\mu\left(Y^{\prime}\right)=1$ because the $Y_{n, m, k}$ and $Z_{n, m}$ are all null sets. Now for $\gamma=\alpha \beta$ with $s(\beta) \in Y^{\prime}$ we have that

$$
\begin{aligned}
U^{\prime}(\gamma) & =c\left(u_{\phi_{\gamma}}\right)_{q(s(\beta))}=c\left(u_{\phi_{\alpha} \circ \phi_{\beta}}\right)_{q(s(\beta))}=c\left(u_{\phi_{\alpha}}\right)_{q(s(\alpha))} c\left(u_{\phi_{\beta}}\right)_{q(s(\beta))} \\
& =U^{\prime}(\alpha) U^{\prime}(\beta),
\end{aligned}
$$

so $U^{\prime}: \mathcal{G} \rightarrow \operatorname{Iso}\left(H^{\prime}\right)$ is a groupoid homomorphism.

$\mathcal{S}$ is almost surely in the kernel of $U^{\prime}$ because, by (2.5), $Y_{n}:=s\left(\mathcal{G}_{\phi_{n}} \cap \mathcal{S}\right) \subset$ $Y$, so for every $n,\left.\phi_{n}\right|_{Y_{n}}=\left.\psi_{n}\right|_{Y_{n}}$ for a $\psi_{n} \in \operatorname{Aut}(\mathcal{S})$. Hence $c\left(u_{\phi_{n}}\right)_{y}=c\left(u_{\psi_{n}}\right)_{y}=$ $\operatorname{id}_{H_{y}}$ for a.e. $y \in Y_{n}$, say for $y \in Y_{n} \backslash Y_{n}^{\prime}$. Now $\mathcal{S}_{Y^{\prime}} \subset \operatorname{ker}\left(U^{\prime}\right)$ for the set $Y^{\prime}=Y \backslash\left(\bigcup Y_{n}^{\prime}\right)$ of full measure. 
Lemma 2.7.4. Let $\xi_{i} \in H^{\prime}$ as in Theorem 2.7.1 be almost central norm one vectors. Then the $\xi_{i}$ are $L^{2}$-almost invariant for $U$.

Proof. Let $\phi \in \operatorname{Aut}(\mathcal{Q})$ be arbitrary and $\left(\phi_{n}\right)_{n \in I}$ as before. Then $q \circ \phi_{n}$ is essentially constant on a.e. $\left(q^{-1}(y), \nu_{y}\right)$ because $\phi_{n}(r(\alpha)) \alpha \in \mathcal{S} \phi_{n}(s(\alpha))$ for all $\alpha \in \mathcal{S}$ and $\left(\left.\mathcal{S}\right|_{q^{-1}(y)}, \nu_{y}\right)$ is ergodic for a.e. $y \in Y$. Hence it can be viewed as a section in $\mathcal{Q}$.

Let $Y_{n}=\left\{y \in Y \mid \phi(y)=q \circ \phi_{n}(y)\right\}$. Then $Y=\bigsqcup_{n \in I} Y_{n}$ since for all $\alpha \in \mathcal{G}$ there exists a unique $n$ such that $\phi_{n}(s(\alpha)) \in \mathcal{S} \alpha$ and if $q\left(\phi_{n}(y)\right)=q\left(\phi_{m}(n)\right)$ we have $\phi_{n}(x) \phi_{m}(x)^{-1} \in \mathcal{S}$ for a.e. $x \in q^{-1}(y)$, hence $\phi_{n}(y) \in \mathcal{S} \phi_{m}(y)$. Now

$$
\begin{aligned}
& \int_{Y}\left\|U(\phi(y)) \xi_{i}(y)-\xi_{i}(\underline{\phi}(y))\right\|_{H_{\underline{\phi}}(y)}^{2} \mathrm{~d} \nu(y) \\
= & \sum_{n \in I} \int_{Y_{n}}\left\|U\left(q\left(\phi_{n}(y)\right)\right) \xi_{i}(y)-\xi_{i}(\underline{\phi}(y))\right\|_{H_{\underline{\phi}}(y)}^{2} \mathrm{~d} \nu(y) \\
= & \sum_{n \in I} \int_{Y_{n}}\left\|\left(u_{\phi_{n}} \xi_{i} u_{\phi_{n}}^{*}\right)(\underline{\phi}(y))-\xi_{i}(\underline{\phi}(y))\right\|_{H_{\underline{\phi}}(y)}^{2} \mathrm{~d} \nu(y) \\
= & \int_{Y}\left\|\left(u_{\phi} \xi_{i} u_{\phi}^{*}\right)(y)-\xi_{i}(y)\right\|_{H_{y}}^{2} \mathrm{~d} \nu(y),
\end{aligned}
$$

which goes to zero for $i \rightarrow \infty$ because the $\xi_{i}$ are almost central.

Remark 2.7.5. [Moo82, Proposition 6.2.2] claims that equivalence relations $\mathcal{R}$ have property (T) iff $L^{\infty}(X) \subset L \mathcal{R}$ has Moore's (T), that is, for bimodules $H$ with

$$
{ }_{L^{\infty}(X)} H_{L \mathcal{R}}={ }_{\lambda \otimes \mathrm{id}}\left(L^{2}(M) \otimes H_{0}\right)_{\rho \otimes \mathrm{id}}
$$

the existence of central vectors follows already from that of almost central vectors that do not have to be $L^{\infty}(X)$-central. We do not see why this is true.

\subsection{Rigidity for groupoid von Neumann alge- bras}

In [Ioa10, Adrian Ioana gives a sufficient condition for $L^{\infty}(X) \subset L \mathcal{G}$ to be rigid.

Proposition 2.8.1 ([Ioa10, Proposition 2.2]). Let $\mathcal{G}$ be an ergodic discrete p.m.p. groupoid such that there exists no sequence of measures $\nu_{n} \in \mathcal{M}(X \times X)$ such that

i) $p_{*}^{i} \nu_{n}=\mu$ for $i=1,2$,

ii) $\lim _{n \rightarrow \infty} \int_{X \times X}\left(f_{1} \otimes f_{2}\right) d \nu_{n}=\int_{X} f_{1} f_{2} d \mu$ for all $f_{1}, f_{2} \in L^{\infty}(X)$,

iii) $\lim _{n \rightarrow \infty}\left\|(\theta \times \theta)_{*} \nu_{n}-\nu_{n}\right\|=0$ for all $\theta \in[\mathcal{G}]$ and 
iv) $\nu_{n}(\Delta)=0$,

where $\Delta$ is the diagonal in $X \times X$. Then $L^{\infty}(X) \subset L \mathcal{G}$ is rigid.

Ioana formulates this only for equivalence relations, but the above theorem is what he actually shows. He also proves that if $\mathcal{G}$ is as in Example 2.1.1, the other direction is true too: If $L^{\infty}(X) \subset L^{\infty}(X) \rtimes \Gamma$ is rigid, then there are no measures as in Proposition 2.8.1.

Together with Theorem 2.7.1 and Proposition 2.5.5 this gives a condition in terms of the groupoid that implies that $L \mathcal{G}$ has property $(\mathrm{T})$ and a characterization when $L^{\infty}(X) \rtimes \Gamma$ has property $(\mathrm{T})$ :

Theorem 2.8.2. If $\mathcal{G}$ is an ergodic discrete p.m.p. groupoid with property $(T)$ such that almost all $\mathcal{G}_{x}^{x}$ are i.c.c. and such that there exists no sequence of measures $\nu_{n} \in \mathcal{M}(X \times X)$ such that

i) $p_{*}^{i} \nu_{n}=\mu$ for $i=1,2$,

ii) $\lim _{n \rightarrow \infty} \int_{X \times X}\left(f_{1} \otimes f_{2}\right) d \nu_{n}=\int_{X} f_{1} f_{2} d \mu$ for all $f_{1}, f_{2} \in L^{\infty}(X)$,

iii) $\lim _{n \rightarrow \infty}\left\|(\theta \times \theta)_{*} \nu_{n}-\nu_{n}\right\|=0$ for all $\theta \in[\mathcal{G}]$ and

iv) $\nu_{n}(\Delta)=0$,

then $L \mathcal{G}$ has property $(T)$.

$L^{\infty}(X) \rtimes \Gamma$ has property (T) if and only if the groupoid $X \rtimes \Gamma$ has property $(T)$ and the above conditions are fulfilled.

Question 2.8.3. Is the above sufficient condition for $L \mathcal{G}$ to have property $(\mathrm{T})$ also necessary for arbitrary groupoids?

Remark 2.8.4. It follows from [Lup17, Theorem 3.5] that for $\mathcal{S} \subset \mathcal{G}$ discrete p.m.p. groupoids with common unit space $X, L \mathcal{S} \subset L \mathcal{G}$ is rigid if and only if $\mathcal{S} \subset \mathcal{G}$ has relative property $(\mathrm{T})$ and $L^{\infty}(X) \subset L \mathcal{G}$ is rigid. Hence for the question how to characterize property $(\mathrm{T})$ of $L \mathcal{G}$ in terms of the groupoid it is enough to study rigidity of the inclusion $L^{\infty}(X) \subset L \mathcal{G}$. 


\section{Chapter 3}

\section{Disintegration rigidity for invariant random positive definite functions}

In this chapter we define invariant random positive definite functions on discrete groups and prove a rigidity result for them. As invariant random positive definite functions will generalize both characters and Invariant Random Subgroups we first collect some information about these in the next two sections.

\subsection{Characters on discrete groups}

Let $\Gamma$ be a discrete, countable group.

Definition 3.1.1. A function $\phi: \Gamma \rightarrow \mathbb{C}$ is called positive definite if for all $g_{1}, \ldots, g_{n} \in \Gamma$ the matrix $\left[\phi\left(g_{j}^{-1} g_{i}\right)\right] \in \mathbb{M}_{n}(\mathbb{C})$ is positive or, equivalently, if $\phi$ induces a state on $\mathbb{C} \Gamma$.

Definition 3.1.2. A character $\tau \in \mathrm{Ch}(\Gamma)$ is a conjugation-invariant positive definite function on $\Gamma$ normalized by $\tau(e)=1$.

A character is called extremal if it is not a non-trivial convex combination of two different characters.

Theorem 3.1.3 ([Tho64b]). The characters of a given group $\Gamma$ form a Choquet simplex, i.e. every character can be uniquely decomposed as a convex combination of extremal ones.

If $\phi$ is a positive definite function, then $\langle g, h\rangle=\phi\left(h^{*} g\right)$ for $g, h \in \Gamma$ extends to a prescalar product on $\mathbb{C} \Gamma$. Let $H$ be the separated completion and denote the image of $\delta_{g}$ in $H$ again by $\delta_{g}$. Then $\pi(g): \delta_{h} \mapsto \delta_{g h}$ extends uniquely to a unitary operator $\pi(g) \in U(H)$. We get a unitary representation $\pi: \Gamma \rightarrow U(H)$ such that $\delta_{e} \in H$ is cyclic and

$$
\phi(g)=\left\langle\pi(g) \delta_{e}, \delta_{e}\right\rangle
$$


for all $g \in \Gamma$. The triple $\left(H, \pi, \delta_{e}\right)$ is unique with these properties up to a unitary. This is called the GNS construction of $\phi$. Sometimes we will also call the von Neumann algebra $\pi(\Gamma)^{\prime \prime} \subset B(H)$ the GNS construction of $\phi$.

If $\phi=\tau$ is a character, its GNS construction is a finite von Neumann algebra with trace extending the character. We denote this trace again by $\tau$ and get $L^{2}\left(\pi(\Gamma)^{\prime \prime}, \tau\right)=H$. In this case we also have a unitary right representation

$$
\rho: \Gamma \rightarrow U(H), \quad \rho(g): \delta_{h} \mapsto \delta_{h g^{-1}} .
$$

Restricted to $\pi(\Gamma)^{\prime \prime} \subset L^{2}\left(\pi(\Gamma)^{\prime \prime}, \tau\right)$, the maps $\pi(g)$ and $\rho(g)$ correspond to $x \mapsto \pi(g) x$ and $x \mapsto x \pi\left(g^{-1}\right)$ when $x$ is viewed as an operator $x \in B(H)$. In particular,

$$
\Gamma \rightarrow \operatorname{Aut}\left(\pi(\Gamma)^{\prime \prime}\right), \quad g \mapsto\left(x \mapsto \pi(g) x \pi\left(g^{-1}\right)\right),
$$

is a well-defined, trace-preserving action.

In the case of the regular character $\delta_{e}$ we get the group von Neumann algebra $L \Gamma$ as GNS construction.

Theorem 3.1.4 ([Tho64b]). Let $\tau$ be a character. Then it is extremal if and only if $\pi(\Gamma)^{\prime \prime} \subset B(H)$ is a factor.

Definition 3.1.5. We say a character is of a certain type if its GNS construction is of this type in the sense of Definition 1.2.29.

Since the GNS construction of a character is finite, its type can only be $I_{1}$ or $I I_{1}$ (or a combination if it is not extremal).

The following theorem characterizes all characters on $S_{\infty}$.

Theorem 3.1.6 ([Tho64a $]$ ). Every extremal character on $S_{\infty}$ is of the form

$$
\tau_{\alpha, \beta}(g)=\prod_{k \geq 2} s_{k}^{r_{k}(g)},
$$

where $r_{k}(g)$ is the number of cycles of length $k$ in $g, \alpha=\left(\alpha_{n}\right)_{n \in \mathbb{N}}$ and $\beta=$ $\left(\beta_{n}\right)_{n \in \mathbb{N}}$ are sequences with $\alpha_{n} \geq \alpha_{n+1} \geq 0$ and $\beta_{n} \geq \beta_{n+1} \geq 0$ for all $n \in \mathbb{N}$ and such that

$$
\sum_{n \in \mathbb{N}} \alpha_{n}+\sum_{n \in \mathbb{N}} \beta_{n} \leq 1
$$

and the $s_{k}$ are given by

$$
s_{k}:=\sum_{n \in \mathbb{N}} \alpha_{n}^{k}+(-1)^{k+1} \sum_{n \in \mathbb{N}} \beta_{n}^{k} .
$$

All such $\tau_{\alpha, \beta}$ are extremal characters and $\tau_{\alpha, \beta}=\tau_{\alpha^{\prime}, \beta^{\prime}}$ implies $\alpha=\alpha^{\prime}$ and $\beta=\beta^{\prime}$.

All extremal characters on $S_{\infty}$ exept for the trivial character and the alternating character are of type II. 
Remark 3.1.7. In the theorem the trivial character belongs to $\alpha=(1,0,0, \ldots)$ and $\beta=0$, the alternating character belongs to $\alpha=0$ and $\beta=(1,0,0, \ldots)$ and the regular character belongs to $\alpha=\beta=0$.

In contrast to $S_{\infty}$, higher rank lattices have only one type $I I$ character:

Theorem 3.1.8 ([Pet14]). Let $\Gamma$ be an irreducible lattice in a higher-rank semisimple Lie group $G$ with trivial center, property $(T)$ and no compact factors. Then every extremal character $\tau \in \mathrm{Ch}(\Gamma)$ is of type $I$ or the regular character $\tau=\delta_{e}$.

Lemma 3.1.9 (Margulis). A group $\Gamma$ as in Theorem 3.1.8 is i.c.c. (hence $\delta_{e}$ is extremal).

Proof. Let $N$ be the union of all finite conjugacy classes. Then $N$ is normal in $\Gamma$. Hence by Margulis' normal subgroup theorem $N$ is trivial or of finite index. If it is trivial, then $\Gamma$ is i.c.c..

As $\Gamma$ is infinite with property $(\mathrm{T})$ it is not amenable and hence not virtually solvable. Hence by Tits' alternative we get $\mathbb{F}_{2} \subset \Gamma$. Clearly, $\mathbb{F}_{2} \cap N=\{e\}$, so $\mathbb{F}_{2}$ maps injectively into the quotient, which implies $[\Gamma: N]=\infty$.

\subsection{Invariant Random Subgroups}

The name "invariant random subgroup" in the following definition is due to AGV14. However, the concept is much older and was, for example, studied by Vershik in the $80 \mathrm{~s}$ and by Stuck-Zimmer in the $90 \mathrm{~s}$. Invariant random subgroups are a tool to study actions, but also behave similarly to normal subgroups.

Definition 3.2.1. An invariant random subgroup (IRS) is a map given by

$$
\varphi: \Omega \rightarrow \operatorname{Sub}(\Gamma), \quad \omega \mapsto \operatorname{Stab}(\omega)=\{\gamma \in \Gamma \mid \gamma \cdot \omega=\omega\}
$$

for a measure preserving action $\Gamma \curvearrowright(\Omega, \mu)$ on a standard probability space.

In fact, invariant random subgroups were originally defined as conjugation invariant measures on $\operatorname{Sub}(\Gamma)$. One can show that this is equivalent to the above definition [AGV14, Proposition 13]. We use this formulation because it will fit with our definition of invariant random positive definite functions and makes our notation easier.

If $\varphi: \Omega \rightarrow \operatorname{PD}(\Gamma)$ is an IRS,

$$
\mathbb{E}[\varphi]: \gamma \mapsto \mu(\{\omega \mid \gamma \cdot \omega=\omega\})
$$

is a character. 
Example 3.2.2. Let $\Omega=\{1, \ldots, n\}$, let $\mu$ be the normalized counting measure and $\Gamma=S_{n}$ the symmetric group. Then $\varphi(i)=\{\sigma \mid \sigma(i)=i\}$ for $i \in \Omega$ is an IRS where $\mathbb{E}[\varphi]=\operatorname{tr}$ is the normalized trace on matrices. The trace tr is not an extremal character on $S_{n}$.

Theorem 3.2.3 (Stuck-Zimmer Theorem, [SZ94]). Let $\Gamma$ be an irreducible lattice in a higher-rank semi-simple Lie group $G$ with trivial center, property (T) and no compact factors. Then every ergodic IRS on an infinite probability space is trivial (i.e., the action is essentially free).

Theorem 3.1.8 implies Theorem 3.2.3 [PT16, Theorem 3.2].

Definition 3.2.4. An action $\Gamma \curvearrowright(\Omega, \mu)$ is totally nonfree if the $\sigma$-algebra generated by the sets of fixed points $\sigma\left(\left\{\Omega_{g} \mid g \in \Gamma\right\}\right)$ coincides with the whole $\sigma$-algebra on $\Omega$.

Theorem 3.2.5 ([Ver10]). If $\varphi$ is an ergodic, totally nonfree IRS, the GNS construction of $\mathbb{E}[\varphi]$ is a factor, hence $\mathbb{E}[\varphi]$ is an extremal character.

We give a detailed proof as there is only a sketch in [Ver10].

Proof. Let $\mathcal{R} \subset \Omega \times \Omega$ be the orbit equivalence relation of $\Gamma \curvearrowright(\Omega, \mu)$. We have a measure $\mu_{\mathcal{R}}$ on $\mathcal{R}$ as defined in Section 2.1 and for $g \in \Gamma$ we define $u_{g} \in U\left(L^{2}\left(\mathcal{R}, \mu_{\mathcal{R}}\right)\right)$ to be given by $u_{g} f(x, y)=f\left(g^{-1} \cdot x, y\right)$. If $\Delta$ denotes the diagonal of $\Omega \times \Omega$ and $\chi_{\Delta} \in L^{2}(\mathcal{R})$ its characteristic function, we have

$$
\left\langle u_{g} \chi_{\Delta}, \chi_{\Delta}\right\rangle=\mu\left(\Omega_{g}\right)=\mathbb{E}[\varphi](g) .
$$

Hence for $H=\overline{\operatorname{span}}\left\{u_{g} \chi_{\Delta}\right\}, M=\left\{u_{g}\right\}^{\prime \prime} \subset B(H)$ is the GNS construction of $\mathbb{E}[\varphi]$. We show that $M=L \mathcal{R}$, which is a factor because the action is ergodic.

As $L \mathcal{R}$ is generated by $\left\{u_{g} \mid g \in \Gamma\right\}$ and $L^{\infty}(\Omega, \mu)$ we have to show that $L^{\infty}(\Omega, \mu) \subset M$. For each $u_{g} \in M$ the spectral projection $\chi_{\{1\}}\left(u_{g}\right)$ also lies in $M$. This is the projection in $L^{\infty}(\Omega)$ onto $\Omega_{g}$. As the fixed points sets generate the $\sigma$-algebra, these projections generate $L^{\infty}(\Omega)$.

For $\Gamma=S_{\infty}$, many characters arise in this way.

Theorem 3.2.6 ([VK81]). Using the notation of Theorem 3.1.6, assume $\beta=$ 0 , let

$$
\delta=1-\sum_{n \in \mathbb{N}} \alpha_{n}
$$

and let $\mathcal{Q}=\mathbb{N} \sqcup[0, \delta]$ with probability measure $\mu$ which is $\left(\alpha_{n}\right)_{n \in \mathbb{N}}$ on $\mathbb{N}$ and the Lebesgue measure on $[0, \delta]$. Let $\Omega=\prod_{1}^{\infty} \mathcal{Q}$ with measure $m_{\alpha, 0}=\prod_{1}^{\infty} \mu$ and let $S_{\infty}$ act on $\left(\Omega, m_{\alpha, 0}\right)$ by permutation of the coordinates.

Then $\tau_{\alpha, 0}=\mathbb{E}[\varphi]$ for this $\operatorname{IRS} \varphi$. 


\subsection{Definition and examples of invariant ran- dom positive definite functions}

The following definition was given by Miklós Abért, Vadim Alekseev and Andreas Thom in 2016.

Definition 3.3.1. Let $\Gamma$ be a discrete group. An invariant random positive definite function (i.r.p.d.f. ) is a measurable $\Gamma$-equivariant map

$$
\varphi: \Omega \rightarrow \mathrm{PD}(\Gamma)
$$

where $(\Omega, \mu)$ is a standard probabilitiy space with a measure preserving $\Gamma$ action and $\operatorname{PD}(\Gamma)$ are the positive definite functions $\phi$ on $\Gamma$ with $\phi(e)=1$ and $\Gamma$-action given by $(g . \phi)(h)=\phi\left(g^{-1} h g\right)$ for $\phi \in \mathrm{PD}(\Gamma)$.

We often write $\varphi_{\omega}$ for $\varphi(\omega)$.

Definition 3.3.2. An i.r.p.d.f. $\varphi$ is called ergodic if the action $\Gamma \curvearrowright(\Omega, \mu)$ is ergodic.

We say $\varphi$ is extremal if $\varphi=c \varphi_{1}+(1-c) \varphi_{2}$ for i.r.p.d.f.'s $\varphi_{i}: \Omega \rightarrow \operatorname{PD}(\Gamma)$ and $c \in(0,1)$ implies that $\varphi_{1}=\varphi_{2}=\varphi$.

When viewing the i.r.p.d.f.'s with given $\Gamma \curvearrowright \Omega$ as $\Gamma$-equivariant positive definite functions $\varphi: \Gamma \rightarrow L^{\infty}(\Omega, \mu)$, they form a compact convex subset of $\ell^{\infty}\left(\Gamma, L^{\infty}(\Omega, \mu)\right)$ with the topology of pointwise weak* convergence. By the Krein-Milman Theorem, the space of these functions is then equal to the closed convex hull of its extremal points. Hence as for characters, every i.r.p.d.f. is the convex integral of extremal i.r.p.d.f.'s.

Example 3.3.3. Invariant Random Subgroups are i.r.p.d.f.'s because the subgroups $\operatorname{Sub}(\Gamma)$ of $\Gamma$ are canonically embedded in $\operatorname{PD}(\Gamma)$ by taking the characteristic function and the stabilizers of an action fulfill the invariance condition in Definition 3.3.1

As for Invariant Random Subgroups, if $\varphi: \Omega \rightarrow \operatorname{PD}(\Gamma)$ is an i.r.p.d.f. ,

$$
\mathbb{E}[\varphi]=\int_{\Omega} \varphi_{\omega} d \mu(\omega)
$$

is a character.

Question 3.3.4. Does extremality of $\varphi$ imply that $\mathbb{E}[\varphi]$ is extremal as a character?

Example 3.3.5. Let $(S, \lambda)$ be the unit sphere in $\mathbb{C}^{n}$ with Lebesgue measure and let $\Gamma$ be a discrete subgroup of the unitary group $U(n)$ acting on $S$ in the natural way. Then

$$
\varphi: S \rightarrow \operatorname{PD}(\Gamma), \quad \varphi_{\xi}(\gamma)=\langle\gamma \cdot \xi, \xi\rangle \quad \forall \xi \in S, \gamma \in \Gamma
$$


is an i.r.p.d.f. for which $\mathbb{E}[\varphi]=\operatorname{tr}$ is the normalized trace on matrices, which is an extremal character on $\Gamma$ iff $\Gamma$ generates $\mathbb{M}_{n}(\mathbb{C})$ as an algebra. For such $\Gamma$, $\varphi$ is an extremal i.r.p.d.f..

Example 3.3.6. Let $\left(S^{1}, \lambda\right)$ be the circle with Lebesgue measure and trivial action of $\mathbb{Z}$. Then

$$
\varphi: S^{1} \rightarrow \mathrm{PD}(\mathbb{Z}), \quad \varphi_{z}(n)=z^{n}
$$

is an i.r.p.d.f. with $\mathbb{E}[\varphi]=\delta_{e}$. Here $\delta_{e}$ is not extremal and $\varphi_{z}$ is an extremal character for every $z \in S^{1}$. In this way every decomposition of a non-extremal character into extremal ones gives an i.r.p.d.f. with trivial action.

Example 3.3.7. Let $G$ be a compact group with Haar measure $\mu$ and $\Gamma<$ $G$. Let $\Gamma$ act on $G$ by left multiplication. Let $\pi: G \rightarrow U(H)$ be a unitary representation and $\xi \in H$ a unit vector. Then

$$
\varphi^{\xi}:(G, \mu) \rightarrow \operatorname{PD}(\Gamma), \quad \varphi_{g}^{\xi}(h)=\langle\pi(h g) \xi, \pi(g) \xi\rangle
$$

is an i.r.p.d.f.. If $\pi: G \rightarrow U\left(\mathbb{C}^{n}\right)$ is irreducible and $\Gamma$ is dense, then $\mathbb{E}\left[\varphi^{\xi}\right](\gamma)=$ $\operatorname{tr}(\pi(\gamma))$, which is an extremal character on $\Gamma$, and $\varphi^{\xi}$ is ergodic and extremal.

Example 3.3 .7 shows that, in contrast to the situation for characters, the decomposition of an i.r.p.d.f. into extremal i.r.p.d.f.'s is not unique: Take an irreducible representation $\pi: G \rightarrow U\left(\mathbb{C}^{n}\right)$, an orthonormal basis $\left(\xi_{i}\right)$ of $\mathbb{C}^{n}$ and $\Gamma<G$ dense. Then

$$
\sum_{i=1}^{n} \frac{1}{n} \varphi^{\xi_{i}} \equiv \operatorname{tr} \circ \pi
$$

For different bases we get different $\varphi^{\xi_{i}}$ 's, so this gives different convex decompositions of the constant i.r.p.d.f. tro $\pi$ into extremal i.r.p.d.f.'s.

Theorem 3.3.8 (VK81], Theorem 3). In the notation of Theorem 3.1.6, let

$$
\delta=1-\sum_{n \in \mathbb{N}} \alpha_{n}-\sum_{n \in \mathbb{N}} \beta_{n},
$$

$\mathbb{N}_{+}=\mathbb{N}_{-}=\mathbb{N}$ and $\mathcal{Q}=\mathbb{N}_{+} \sqcup \mathbb{N}_{-} \sqcup[0, \delta]$ with the probability measure $\mu$ which is $\left(\alpha_{n}\right)_{n \in \mathbb{N}}$ on $\mathbb{N}_{+},\left(\beta_{n}\right)_{n \in \mathbb{N}}$ on $\mathbb{N}_{-}$and the Lebesgue measure on $[0, \delta]$. Then let $\Omega=\prod_{1}^{\infty} \mathcal{Q}$ with the measure $m_{\alpha, \beta}=\prod_{1}^{\infty} \mu$ and let $S_{\infty}$ act on $\left(\Omega, m_{\alpha, \beta}\right)$ by permutation of the coordinates.

For $g \in S_{\infty}$ and $\omega \in \Omega$ define $\operatorname{sgn}(g, \omega)$ to be 1 if

$$
\prod_{(i, j): \omega_{i}, \omega_{j} \in \mathbb{N}_{-}, i<j}(g(j)-g(i))
$$

is positive and -1 otherwise. This fulfills the cocycle identity

$$
\operatorname{sgn}(g h, \omega)=\operatorname{sgn}(h, \omega) \operatorname{sgn}(g, h . \omega) .
$$


Let

$$
\varphi_{\omega}(g)= \begin{cases}\operatorname{sgn}(g, \omega) & \text { if } g \cdot \omega=\omega, \\ 0 & \text { if } g \cdot \omega \neq \omega .\end{cases}
$$

Then $\tau_{\alpha, \beta}=\mathbb{E}[\varphi]$.

The following theorem proves that the above $\varphi$ is an i.r.p.d.f.. If $\beta$ is non-trivial, then $\varphi$ is not an IRS.

Theorem 3.3.9. Let $\Gamma \curvearrowright(\Omega, \mu)$ be a p.m.p. action and $c: \Gamma \times \Omega \rightarrow S^{1} a$ cocycle as in (3.1). Then

$$
\varphi_{\omega}(g)= \begin{cases}c(g, \omega) & \text { if } g \cdot \omega=\omega, \\ 0 & \text { if } g \cdot \omega \neq \omega,\end{cases}
$$

is an i.r.p.d.f..

If $c$ is not constantly $1, \varphi$ is not an IRS because it takes values outside $\{0,1\}$.

Proof. To show that $\varphi$ is invariant we need that $c(g, h \omega)=c\left(h^{-1} g h, \omega\right)$ if $h^{-1}$ gh. $\omega=\omega$. By the cocycle identity we have

$$
1=c(1, \omega)=c\left(h^{-1} h, h^{-1} g h . \omega\right)=c(h, \omega) c\left(h^{-1}, g h . \omega\right)
$$

and hence

$$
c\left(h^{-1} g h, \omega\right)=c(h, \omega) c\left(h^{-1} g, h . \omega\right)=c(h, \omega) c(g, h . \omega) c\left(h^{-1}, g h . \omega\right)=c(g, h . \omega) .
$$

Now we show that $\varphi_{\omega}$ is positive definite for a.e. $\omega \in \Omega$. Let $\mathcal{R} \subset \Omega \times \Omega$ be the orbit equivalence relation of $\Gamma \curvearrowright(\Omega, \mu)$, equipped with the measure as in Chapter 2. Then $\pi: \Gamma \rightarrow U\left(L^{2}(\mathcal{R})\right)$, given by

$$
(\pi(g) \xi)(x, y)=c(g, x) \xi(g . x, y)
$$

is a unitary representation and for every $X \subset \Omega$ we find a vector $\xi_{X}=$ $\chi_{\{(x, x) \mid x \in X\}} \in L^{2}(\mathcal{R})$ such that

$$
\int_{X} \varphi_{\omega}(g)=\left\langle\pi(g) \xi_{X}, \xi_{X}\right\rangle
$$

Hence for every $a \in \mathbb{C} \Gamma$ we have

$$
\int_{X} \varphi_{\omega}\left(a^{*} a\right) \geq 0
$$

for all $X \subset \Omega$ and hence $\varphi_{\omega}\left(a^{*} a\right) \geq 0$ almost everywhere.

The i.r.p.d.f.'s in Theorem 3.3.9 are supported on an IRS in the sense that $\varphi_{\omega}(\gamma)=0$ if $\gamma \cdot \omega \neq \omega$.

Question 3.3.10. Is every i.r.p.d.f. $\varphi$ such that $\mathbb{E}[\varphi]$ is of type II supported on an IRS ? 


\subsection{Invariant random positive definite func- tions and von Neumann algebras}

In this section we translate i.r.p.d.f.'s into the language of von Neumann algebras in order to be able to use von Neumann methods to study them in the next section.

Fix a discrete group $\Gamma$, a character $\tau \in \operatorname{Ch}(\Gamma)$ and an ergodic, measure preserving action $\alpha: \Gamma \curvearrowright(\Omega, \mu)$ on a standard probability space. Let $A:=$ $L^{\infty}(\Omega, \mu)$ and write again $\alpha$ for the corresponding action on $A$. Let $\pi: \Gamma \rightarrow$ $U(H)$ be the GNS representation of $\tau$.

Lemma 3.4.1. Let $\varphi$ be an i.r.p.d.f. with $\mathbb{E}[\varphi]=\tau$ and for each $\omega \in \Omega$ let $\left(\pi_{\omega}, H_{\omega}, \xi_{\omega}\right)$ be the GNS construction of $\varphi_{\omega}$. Let

$$
H_{\varphi}:=\int_{\Omega}^{\oplus} H_{\omega} d \mu(\omega)
$$

be the direct integral of Hilbert spaces, $\xi=\left(\xi_{\omega}\right)_{\omega \in \Omega} \in H_{\varphi}$ and

$$
\pi_{\varphi}=\int_{\Omega}^{\oplus} \pi_{\omega} d \mu(\omega): \Gamma \rightarrow B\left(H_{\varphi}\right)
$$

the direct integral of representations. Then $\pi_{\varphi}(\Gamma)^{\prime \prime} \cong \pi(\Gamma)^{\prime \prime}$ with isomorphism taking $\pi_{\varphi}(\gamma)$ to $\pi(\gamma)$ for all $\gamma \in \Gamma$.

Proof. Let $p \in B\left(H_{\varphi}\right)$ be the orthogonal projection onto the cyclic representation of $\xi$. Then $p \in \pi_{\varphi}(\Gamma)^{\prime}$. As $\mathbb{E}[\varphi]=\tau$ we have

$$
\left\langle\pi_{\varphi}(\gamma) \xi, \xi\right\rangle=\int_{\Omega}\left\langle\pi_{\omega}(\gamma) \xi_{\omega}, \xi_{\omega}\right\rangle d \mu(\omega)=\int_{\Omega} \varphi_{\omega}(\gamma) d \mu(\omega)=\tau(\gamma)
$$

for all $\gamma \in \Gamma$. So $\left(p\left(H_{\varphi}\right), \pi_{\varphi}, \xi\right)$ is a GNS triple for $\tau$ and therefore by uniqueness of the GNS construction

$$
\pi(\Gamma)^{\prime \prime} \cong\left(p \pi_{\varphi}(\Gamma) p\right)^{\prime \prime}=p\left(\pi_{\varphi}(\Gamma)\right)^{\prime \prime}
$$

with isomorphism taking $\pi(\gamma)$ to $p \pi_{\varphi}(\gamma)$ for all $\gamma \in \Gamma$. Now we show that

$$
\Phi:\left(\pi_{\varphi}(\Gamma)\right)^{\prime \prime} \rightarrow p\left(\pi_{\varphi}(\Gamma)\right)^{\prime \prime}, x \mapsto p x,
$$

is an isomorphism. It is clearly a surjective homomorphism. For injectivity let $x \in\left(\pi_{\varphi}(\Gamma)\right)^{\prime \prime}$ with $\Phi\left(x^{*} x\right)=p x^{*} x p=0$. Then for all $a \in \mathbb{C} \Gamma$ we have

$$
0=\left\langle x^{*} x \pi_{\varphi}(a) \xi, \pi_{\varphi}(a) \xi\right\rangle=\int_{\Omega}\left\langle\left(x^{*} x\right)_{\omega} \pi_{\omega}(a) \xi_{\omega}, \pi_{\omega}(a) \xi_{\omega}\right\rangle d \mu(\omega)
$$

and therefore $\left\langle\left(x^{*} x\right)_{\omega} \pi_{\omega}(a) \xi_{\omega}, \pi_{\omega}(a) \xi_{\omega}\right\rangle=0$ for almost all $\omega \in \Omega$. But $\pi_{\omega}(\mathbb{C} \Gamma) \xi_{\omega}$ is dense in $H_{\omega}$, so $\left(x^{*} x\right)_{\omega}=0$ for almost all $\omega$. Hence $x=0$ and $\Phi$ is injective.

Composing the two isomorphisms we get $\pi(\Gamma)^{\prime \prime} \cong p \pi_{\varphi}(\Gamma)^{\prime \prime} \cong \pi_{\varphi}(\Gamma)^{\prime \prime}$ with isomorphism mapping $\pi(\gamma)$ to $\pi_{\varphi}(\gamma)$. 
Lemma 3.4.2. Let $M:=\left(A \cup \pi_{\varphi}(\Gamma)\right)^{\prime \prime}=\int_{\Omega}^{\oplus} \pi_{\omega}(\Gamma)^{\prime \prime} d \mu(\omega)$. Then $M$ is a finite von Neumann algebra.

Proof. Let $u \in M$ be such that $u^{*} u=1$. By the Kaplansky Density Theorem we find a sequence of finite sums

$$
t_{n}=\sum_{i} p_{n, i} x_{n, i}
$$

converging to $u$ in the strong* topology such that $\left\|t_{n}\right\| \leq 1$ for all $n, p_{n, i} \in A$ are mutually orthogonal projections for fixed $n$ and $x_{n, i} \in \pi_{\varphi}(\Gamma)^{\prime \prime}$. We then have $t_{n}^{*} t_{n} \stackrel{s^{*}}{\rightarrow} 1$ since the strong* topology is jointly continuous on bounded sets. Hence $\left|t_{n}\right| \stackrel{s^{*}}{\rightarrow} 1$ by [Tak02, Lemma II.4.6]. Letting

$$
f(t):= \begin{cases}1-2 t, & 0 \leqslant t \leqslant 1 / 2 \\ 0, & 1 / 2 \leqslant t \leqslant 1\end{cases}
$$

we obtain (again by [Tak02, Lemma II.4.6]) $f\left(\left|t_{n}\right|\right) \stackrel{s^{*}}{\rightarrow} 0$, and therefore $\left|t_{n}\right|+$ $f\left(\left|t_{n}\right|\right) \stackrel{s^{*}}{\rightarrow} 1$. However, as $1 / 2 \leqslant t+f(t) \leqslant 1$ on $[0,1]$, we also have $1 / 2 \leqslant$ $\left|t_{n}\right|+f\left(\left|t_{n}\right|\right) \leqslant 1$

Let $t_{n}=u_{n}\left|t_{n}\right|$ be the polar decomposition of $t_{n}$. Then we have

$$
u_{n}\left(\left|t_{n}\right|+f\left(\left|t_{n}\right|\right)\right) \stackrel{s^{*}}{\rightarrow} u
$$

because $f\left(\left|t_{n}\right|\right) \stackrel{s^{*}}{\rightarrow} 0$. On the other hand, $\left|t_{n}\right|+f\left(\left|t_{n}\right|\right)$ is invertible with the inverse bounded by 2 and $\left(\left|t_{n}\right|+f\left(\left|t_{n}\right|\right)\right)^{-1} \stackrel{s^{*}}{\rightarrow} 1$ again by Tak02, Lemma II.4.6]. Therefore,

$$
u_{n}=u_{n}\left(\left|t_{n}\right|+f\left(\left|t_{n}\right|\right)\right)\left(\left|t_{n}\right|+f\left(\left|t_{n}\right|\right)\right)^{-1} \stackrel{s^{*}}{\rightarrow} u .
$$

Let $x_{n, i}=v_{n, i}\left|x_{n, i}\right|$ be the polar decomposition of $x_{n, i}$. Then

$$
u_{n}=\sum_{i} p_{n, i} v_{n, i}
$$

because using that $A$ commutes with $\pi_{\varphi}(\Gamma)^{\prime \prime}$ and that the $p_{n, i}$ are mutually orthogonal we get that

$$
\left|t_{n}\right|=\sum_{i} p_{n, i}\left|x_{n, i}\right|
$$

and hence

$$
\left(\sum_{i} p_{n, i} v_{n, i}\right)\left|t_{n}\right|=\sum_{i} p_{n, i} v_{n, i}\left|x_{n, i}\right|=\sum_{i} p_{n, i} x_{n, i}=t_{n} .
$$


Now (3.3) and (3.2) imply that

$$
u_{n}^{*} u_{n}=\sum_{i} p_{n, i} v_{n, i}^{*} v_{n, i} \stackrel{s^{*}}{\rightarrow} 1,
$$

and therefore

$$
\sum_{i} p_{n, i} \stackrel{s^{*}}{\rightarrow} 1
$$

Since $\pi_{\varphi}(\Gamma)^{\prime \prime} \cong \pi(\Gamma)^{\prime \prime}$ is finite, there exist partial isometries $w_{n, i} \in \pi_{\varphi}(\Gamma)^{\prime \prime}$ such that $u_{n, i}=v_{n, i}+w_{n, i}$ are unitaries. Let $q_{n, i}:=w_{n, i}^{*} w_{n, i}$ be the source projections of the $w_{n, i}$. Then

$$
\sum_{i} p_{n, i} q_{n, i}=\sum_{i} p_{n, i}\left(1-v_{n, i}^{*} v_{n, i}\right) \leq 1-\sum_{i} p_{n, i} v_{n, i}^{*} v_{n, i}=1-u_{n}^{*} u_{n} \stackrel{s^{*}}{\rightarrow} 0,
$$

and therefore

$$
\sum_{i} p_{n, i} w_{n, i}=\left(\sum_{i} p_{n, i} w_{n, i}\right)\left(\sum_{i} p_{n, i} q_{n, i}\right) \stackrel{s^{*}}{\rightarrow} 0 .
$$

Thus by 3.3

$$
\sum_{i} p_{n, i} u_{n, i}=u_{n}+\sum_{i} p_{n, i} w_{n, i} \stackrel{s^{*}}{\rightarrow} u
$$

and therefore, since the $u_{n, i}$ are unitaries,

$$
\sum_{i} p_{n, i}=\sum_{i} p_{n, i} u_{n, i} u_{n, i}^{*} \stackrel{s^{*}}{\rightarrow} u u^{*}
$$

Hence $u u^{*}=1$ by (3.4), which means that $M$ is finite.

Lemma 3.4.3. If $\tau$ is extremal, we have $M \cong A \bar{\otimes} \pi_{\varphi}(\Gamma)^{\prime \prime}$ with isomorphism taking $x a \in M$ to $a \otimes x \in A \bar{\otimes} \pi_{\varphi}(\Gamma)^{\prime \prime}$ for all $a \in A$ and $x \in \pi_{\varphi}(\Gamma)^{\prime \prime}$.

Proof. Since $M$ is finite by the previous lemma, there exists a normal faithful conditional expectation $E: M \rightarrow \pi_{\varphi}(\Gamma)^{\prime \prime}$. Since $\pi_{\varphi}(\Gamma)^{\prime \prime}$ and $A$ commute and $E$ is $\pi_{\varphi}(\Gamma)^{\prime \prime}$-linear,

$$
E(a)=E\left(\pi_{\varphi}(\gamma) a \pi_{\varphi}\left(\gamma^{-1}\right)\right)=\pi_{\varphi}(\gamma) E(a) \pi_{\varphi}\left(\gamma^{-1}\right)
$$

for all $\gamma \in \Gamma$ and $a \in A$. Thus, $E(A)$ is contained in the center of $\pi_{\varphi}(\Gamma)^{\prime \prime} \cong$ $\pi(\Gamma)^{\prime \prime}$, which is equal to $\mathbb{C}$ since $\tau$ is extremal. Now the claim follows from [Str81, Theorem 9.12].

On $M \operatorname{resp} . L^{1}(M)$ we define a $\Gamma$-action $\theta$ by

$$
\theta_{\gamma}(a \otimes m)=\alpha_{\gamma}(a) \otimes \pi(\gamma) m \pi\left(\gamma^{-1}\right)
$$

By $M^{\theta}$ resp. $L^{1}(M)^{\theta}$ we denote the elements that are invariant under $\theta$. 
Proposition 3.4.4. Given an ergodic action and an extremal character $\tau \in$ $\mathrm{Ch}(\Gamma)$ there is a one-to-one correspondence between i.r.p.d.f.'s $\varphi: \Omega \rightarrow \operatorname{PD}(\Gamma)$ with $\mathbb{E}[\varphi]=\tau$ and positive selfadjoint elements $f \in L^{1}(M)^{\theta}$ with $\int_{\Omega} f_{\omega} d \mu(\omega)=$ 1 such that

$$
\varphi_{\omega}(\gamma)=\tau\left(\pi(\gamma) f_{\omega}\right)
$$

Proof. By Lemma 3.4.1 and Lemma 3.4.3, we have $\pi(\Gamma)^{\prime \prime} \cong \pi_{\omega}(\Gamma)^{\prime \prime}$ for a.e. $\omega \in \Omega$ with the canonical isomorphism sending $\pi(\gamma)$ to $\pi_{\omega}(\gamma)$ for each $\gamma \in \Gamma$. As $\varphi_{\omega}(\gamma)=\left\langle\pi_{\omega}(\gamma) \xi_{\omega}, \xi_{\omega}\right\rangle$, we can extend it to

$$
\varphi_{\omega}: \pi_{\omega}(\Gamma)^{\prime \prime} \rightarrow \mathbb{C}, x \mapsto\left\langle x \xi_{\omega}, \xi_{\omega}\right\rangle
$$

which is a positive normal functional on $\pi_{\omega}(\Gamma)^{\prime \prime}$ and therefore on $\pi(\Gamma)^{\prime \prime}$. So by Theorem 1.2.38 there exists a unique positive element $f_{\omega} \in L^{1}\left(\pi(\Gamma)^{\prime \prime}, \tau\right)$ such that $\varphi_{\omega}(x)=\tau\left(x f_{\omega}\right)$ for all $x \in \pi(\Gamma)^{\prime \prime}$. Let $f: \Omega \rightarrow L^{1}\left(\pi(\Gamma)^{\prime \prime}\right), \omega \mapsto f_{\omega}$. To see that $f$ is $\theta$-invariant, we calculate

$$
\tau\left(\pi(\gamma) f_{\alpha_{\gamma^{\prime}}(\omega)}\right)=\varphi_{\alpha_{\gamma^{\prime}}(\omega)}(\gamma)=\varphi\left(\gamma^{\prime-1} \gamma \gamma^{\prime}\right)=\tau\left(\pi(\gamma) \pi\left(\gamma^{\prime}\right) f_{\omega} \pi\left(\gamma^{\prime-1}\right)\right)
$$

so $\alpha_{\gamma^{\prime}}^{-1}(f)_{\omega}=f_{\alpha_{\gamma^{\prime}}(\omega)}=\pi\left(\gamma^{\prime}\right) f_{\omega} \pi\left(\gamma^{\prime-1}\right)$ for all $\gamma^{\prime} \in \Gamma$ by uniqueness of $f$, hence $\theta(f)=f$. It follows that $\left\|f_{\omega}\right\|_{1}$ is $\Gamma$-invariant and hence constant, so $f \in L^{1}(M)^{\theta}$. We have for all $\gamma \in \Gamma$

$$
\tau\left(\pi(\gamma) \int f_{\omega} d \mu(\omega)\right)=\int \tau\left(\pi(\gamma) f_{\omega}\right) d \mu(\omega)=\int \varphi_{\omega}(\gamma) d \mu(\omega)=\tau(\pi(\gamma))
$$

hence $\int f_{\omega} d \mu(\omega)=1$. By [Lüc02, Lemma $\left.8.3(3)\right], f$ is a selfadjoint operator.

Conversely it is easy to check that such an $f$ defines an i.r.p.d.f. $\varphi$ with $\mathbb{E}(\varphi)=\tau$ by $\varphi_{\omega}(\gamma)=\tau\left(\pi(\gamma) f_{\omega}\right)$.

Remark 3.4.5. $\quad$ i) If $\varphi$ is as in Example 3.3.5 with $\Gamma$ big enough so that $\varphi$ is extremal, we have $f: S \rightarrow \mathbb{M}_{n}(\mathbb{C})$ with $f_{\xi}$ the orthogonal projection on $\operatorname{span}(\xi)$.

ii) Similarly, if $\Gamma$ in Example 3.3.7 is dense and $\pi$ irreducible, we find $f: G \rightarrow$ $\mathbb{M}_{n}(\mathbb{C})$ where $f_{g}$ is the orthogonal projection on $\operatorname{span}(\pi(g) \xi)$.

iii) The i.r.p.d.f. in Example 3.3.6 is not of the form as in Proposition 3.4.4. Hence the ergodicity and extremality assumptions are necessary (or at least one of them is).

Lemma 3.4.6. In fact, for $f \in L^{1}(M)^{\theta}$ as in Proposition 3.4.4 the condition that $\int_{\Omega} f_{\omega} d \mu(\omega)=1$ is equivalent to $\tau_{M}(f)=1$, where $\tau_{M}=\int_{\Omega} \otimes \tau$ is the trace on $M$. 
Proof. Let $f$ be constructed from $\varphi$ as above. Then

$$
\tau_{M}(f)=\int_{\Omega} \tau\left(f_{\omega}\right) d \mu(\omega)=\int_{\Omega} \varphi_{\omega}(e) d \mu(\omega)=\int_{\Omega} 1 d \mu(\omega)=1 .
$$

For the other direction let first $p \in M^{\theta}$ be a projection. Then

$$
\tau(\gamma)=\tau\left(\pi(\gamma) \int p_{\omega} d \mu(\omega)\right)+\tau\left(\pi(\gamma) \int(1-p)_{\omega} d \mu(\omega)\right)
$$

is a convex decomposition into two characters. So by extremality of $\tau$,

$$
\int p_{\omega} d \mu(\omega)=\tau_{M}(p) \cdot 1
$$

Now let $f \in L^{1}(M)^{\theta}$ be positive selfadjoint with $\tau_{M}(f)=1$. Then it follows from the above and the spectral theorem for $f$ that $\int f_{\omega} d \mu(\omega)=\tau_{M}(f) \cdot 1=1$.

Lemma 3.4.7. For $\tau$ extremal and $\alpha$ ergodic the extremal i.r.p.d.f.'s $\varphi$ given $\alpha$ and $\mathbb{E}[\varphi]=\tau$ correspond to minimal projections in $M^{\theta} . M^{\theta}$ is a direct sum of matrix algebras.

Proof. Let $\varphi: \Omega \rightarrow \operatorname{PD}(\Gamma)$ be an extremal i.r.p.d.f. and $f \in L^{1}(M)^{\theta}$ as in Proposition 3.4.4 such that $\tau\left(f_{\omega} \pi(\gamma)\right)=\varphi_{\omega}(\gamma)$ for a.e. $\omega \in \Omega$ and all $\gamma \in \Gamma$. Assume that $f$ is not a scalar multiple of a projection. Then there is a $c \in \mathbb{R}^{+}$ such that

$$
f^{<c}:=\chi([0, c)) f \quad \text { and } \quad f^{\geq c}:=\chi([c, \infty)) f,
$$

are both nonzero with $\chi(I)$ denoting the spectral projection on $I$. These are again positive elements in $M^{\theta}$ hence $\tau_{M}\left(f^{<c}\right)^{-1} f^{<c}$ and $\tau_{M}\left(f^{\geq c}\right)^{-1} f^{\geq c}$ define two different i.r.p.d.f.'s $\varphi^{<c}$ and $\varphi^{\geq c}$ such that

$$
\varphi=\tau_{M}\left(f^{<c}\right) \varphi^{<c}+\tau_{M}\left(f^{\geq c}\right) \varphi^{\geq c}
$$

contradicting the extremality of $\varphi$. So $f=\tau_{M}(p)^{-1} p$ for some projection $p \in M^{\theta}$. If $p$ is not minimal in $M^{\theta}$, say $q<p$ and $q \in M^{\theta}$, then again $q$ and $p-q$ define two i.r.p.d.f.'s such that a convex combination gives $\varphi$, which contradicts extremality.

Conversely every minimal projection $p \in M^{\theta}$ gives an extremal i.r.p.d.f. $\varphi$ because if there was a decomposition $\varphi=c \varphi_{1}+(1-c) \varphi_{2}$ for some $0<c<1$ and different i.r.p.d.f.'s $\varphi_{i}$, this would give different positive elements $f_{1}, f_{2} \in M^{\theta}$ such that $\tau_{M}(p)^{-1} p=c f_{1}+(1-c) f_{2}$, which is not possible for a minimal projection $p$.

Since the set of i.r.p.d.f.'s is the closed convex hull of its extremal points, every positive trace 1 element of $M^{\theta}$ is a convex integral of minimal projections. This means $M^{\theta}$ is generated by its minimal projections, hence by Theorem 1.2 .30 it is of type I with no diffuse part, i.e., $\mathcal{Z}\left(M^{\theta}\right)=L^{\infty}(X, \mu)$ such that every point in $X$ has positive mass. Since it is also finite, it follows that $M^{\theta}$ is a (maybe infinite) direct sum of matrix algebras. 
Remark 3.4.8. Let $\Gamma, \tau, \alpha$ be fixed as in Proposition 3.4.4. Then

i) As $M^{\theta}$ is a direct sum of matrix algebras every i.r.p.d.f. $\varphi$ with $\mathbb{E}[\varphi]=\tau$ is a convex combination of countably many extremal ones.

ii) $M^{\theta}=\mathbb{C}$ iff the constant i.r.p.d.f. $\tau$ is the only one with $\mathbb{E}[\varphi]=\tau$, i.e., $\tau$ is disintegration rigid. This is also equivalent to the constant $\tau$ being an extremal i.r.p.d.f..

iii) $M^{\theta}$ is abelian iff the decomposition of i.r.p.d.f.'s with $\mathbb{E}[\varphi]=\tau$ into extremal ones is unique.

iv) $M^{\theta}$ is finite-dimensional iff every i.r.p.d.f. is a finite convex sum of extremal ones.

\subsection{Disintegration rigidity of the regular char- acter on i.c.c. groups}

In this section we show the following theorem.

Theorem 3.5.1. Let $\Gamma$ be an i.c.c. group. Let $\varphi: \Omega \rightarrow \operatorname{PD}(\Gamma)$ be an i.r.p.d.f. on $\Gamma$ with $\mathbb{E}[\varphi]=\delta_{e}$. Then $\varphi(\omega)=\delta_{e}$ for almost every $\omega \in \Omega$.

Definition 3.5.2. If the conclusion of the theorem holds, we say $\left(\Gamma, \delta_{e}\right)$ is disintegration rigid.

Remark 3.5.3. Theorem 3.1.6, Remark 3.1.7 and Theorem 3.3.8 show that the regular character, the trivial character and the alternating character are the only disintegration rigid characters on $S_{\infty}$. Indeed, if $S_{\infty} \curvearrowright\left(\Omega, m_{\alpha, \beta}\right)$ is the action from Theorem 3.3 .8 such that $\tau_{\alpha, \beta}$ is none of these three characters, we have $0<\alpha_{1}<1$ or $0<\beta_{1}<1$. Assume w.l.o.g. that $0<\alpha_{1}<1$. Then for every nontrivial $g \in S_{\infty}$ and $j \in \operatorname{supp}(g)=\{j \mid g(j) \neq j\}$

$$
\begin{aligned}
0 & <m_{\alpha, \beta}\left(\left\{\omega \in \Omega \mid \omega_{i}=1 \in \mathbb{N}_{+} \forall i \in \operatorname{supp}(g)\right\}\right) \\
& \leq m_{\alpha, \beta}(\{\omega \in \Omega \mid g \cdot \omega=\omega\}) \\
& \leq 1-m_{\alpha, \beta}\left(\left\{\omega \in \Omega \mid \omega_{j}=1 \in \mathbb{N}_{+}, g \cdot \omega_{j} \neq 1 \in \mathbb{N}_{+}\right\}\right)<1 .
\end{aligned}
$$

Hence the $\varphi$ in Theorem 3.2.6 is non-constant with $\mathbb{E}[\varphi]=\tau_{\alpha, \beta}$.

The trivial and the alternating character are clearly disintegration rigid because every positive definite function takes values in the unit disk, and thus, if an i.r.p.d.f. intergrates to a character which takes values only on the boundary of the unit disk, the i.r.p.d.f. has to be constant.

Definition 3.5.4. A trace-preserving action on a finite von Neumann algebra $\Gamma \rightarrow \operatorname{Aut}(M)$ is called weakly mixing if $\mathbb{C} \hat{1}$ is the only finite-dimensional, $\Gamma$ invariant subspace in $L^{2}(M)$. 
The following lemma might be known to experts.

Lemma 3.5.5. Let $\Gamma$ be an i.c.c. group. Then the conjugation action on $L \Gamma$ is weakly mixing.

Proof. Let $\Gamma=\left\{\gamma_{j} \mid j \in \mathbb{N}\right\}$ be an enumeration of $\Gamma$. Assume $\mathbb{C} \delta_{e} \neq H \subset$ $\ell^{2}(\Gamma)$ is an $\Gamma$-invariant, finite-dimensional subspace and let $\left\{\xi_{1}, \ldots, \xi_{n}\right\}$ be an orthonormal basis of $H$ such that $\xi_{1} \notin \mathbb{C} \delta_{e}$. Then for every $\varepsilon>0$ there is a $K \in \mathbb{N}$ such that

$$
\left\|\xi_{j}-\sum_{i=1}^{K}\left\langle\xi_{j}, \delta_{\gamma_{i}}\right\rangle \delta_{\gamma_{i}}\right\|<\varepsilon \quad \text { for all } j=1, \ldots, n .
$$

Let $F=\left\{\gamma_{1}, \ldots, \gamma_{K}\right\}$. Then by [CSU16, Proposition 3.4] there exists a $\gamma \in \Gamma$ such that

$$
\gamma F \gamma^{-1} \cap F \subset\{e\}
$$

Let $H_{F}:=\operatorname{span}(F)$ and $P_{F}$ the orthogonal projection on $H_{F}$.

As $\left\{\gamma \xi_{1} \gamma^{-1}, \ldots, \gamma \xi_{n} \gamma^{-1}\right\}$ is again an orthonormal basis of $H$ we have $c_{j} \in \mathbb{C}$ with $\sum_{j=1}^{n}\left|c_{j}\right|^{2}=1$ such that

$$
\xi_{1}=\sum_{j=1}^{n} c_{j} \gamma \xi_{j} \gamma^{-1}=\sum_{j=1}^{n} c_{j}\left(\sum_{i=1}^{K}\left\langle\xi_{j}, \delta_{\gamma_{i}}\right\rangle \delta_{\gamma \gamma_{i} \gamma^{-1}}+\sum_{i=K+1}^{\infty}\left\langle\xi_{j}, \delta_{\gamma_{i}}\right\rangle \delta_{\gamma \gamma_{i} \gamma^{-1}}\right) .
$$

We have $\sum_{i=1}^{K}\left\langle\xi_{j}, \delta_{\gamma_{i}}\right\rangle \delta_{\gamma \gamma_{i} \gamma^{-1}} \in H_{F}^{\perp}+\mathbb{C} \delta_{e}$ because of 3.6 , which together with (3.5) implies

$$
\begin{aligned}
\left\|P_{F}\left(\xi_{1}\right)\right\| & \leq\left|\left\langle\xi_{1}, \delta_{e}\right\rangle\right|+\left\|P_{F}\left(\sum_{j=1}^{n} c_{j} \sum_{i=K+1}^{\infty}\left\langle\xi_{j}, \delta_{\gamma_{i}}\right\rangle \delta_{\gamma \gamma_{i} \gamma-1}\right)\right\| \\
& \leq\left|\left\langle\xi_{1}, \delta_{e}\right\rangle\right|+\varepsilon \sum_{j=1}^{n}\left|c_{j}\right| \\
& \leq\left|\left\langle\xi_{1}, \delta_{e}\right\rangle\right|+n \varepsilon
\end{aligned}
$$

Since $\left\|P_{F}\left(\xi_{1}\right)\right\|>1-\varepsilon$ by 3.5 , we get a contradiction when choosing $\varepsilon<$ $n^{-1}\left(1-\left|\left\langle\xi_{1}, \delta_{e}\right\rangle\right|\right)$.

Definition 3.5.6. We call an extremal character conjugation weakly mixing if the conjugation action on its GNS construction is weakly mixing.

Question 3.5.7. Which other characters are conjugation weakly mixing?

The following theorem implies Theorem 3.5.1.

Theorem 3.5.8. Let $\tau$ be a conjugation weakly mixing character on $\Gamma$. Then $(\Gamma, \tau)$ is disintegration rigid. 
Proof. We first assume that $\alpha$ is ergodic. An action on a finite von Neumann algebra $\sigma: \Gamma \curvearrowright N$ is weakly mixing if and only if for every action $\alpha: \Gamma \curvearrowright$ $A$ on a finite von Neumann algebra one has $(A \bar{\otimes} N)^{(\alpha \otimes \sigma)}=A^{\alpha} \otimes 1$ Vae07, Proposition D.2]. So if we take $A=L^{\infty}(\Omega)$ as in Section 3.4 and $N=\pi(\Gamma)^{\prime \prime}$, Lemma 3.5.5 implies that

$$
M^{\theta}=(A \bar{\otimes} N)^{(\alpha \otimes \operatorname{conj}(\pi))}=A^{\alpha}=\mathbb{C} .
$$

$\tau$ is extremal because if the conjugation action is weakly mixing, it must be ergodic, hence the GNS construction is a factor. Hence by Proposition 3.4.4 every i.r.p.d.f. $\varphi$ with $\mathbb{E}[\varphi]=\tau$ is given by an element in $M^{\theta}$, which proves the statement in the ergodic case.

The general case follows by ergodic decomposition: Let $\varphi$ be an i.r.p.d.f. with $\mathbb{E}[\varphi]=\tau$. Then the restriction to the ergodic components defined after Theorem 1.3.17 are ergodic i.r.p.d.f.'s. The expectation values of these ergodic i.r.p.d.f.'s integrate to $\tau$ by 1.3.17 iii) and are therefore by extremality $\mu$ almost surely equal to $\tau$. Hence we can apply the statement to them and get that they are constantly $\tau \nu$-almost surely, which by 1.3.17 iii) implies that $\varphi$ is constantly $\tau \mu$-almost surely. 


\section{Chapter 4}

\section{A rigidity result for regular subfactors}

\subsection{A question about regular subfactors of the von Neumann algebra of lattices in higher- rank groups}

In this chapter we study possible analogues of Margulis' Normal Subgroup Theorem [Mar91, Theorem IX.5.3] in the setting of subfactors.

Theorem 4.1.1 (Margulis' Normal Subgroup Theorem). Let $\Gamma$ be an irreducible lattice in a higher-rank simple Lie group $G$ with trivial center. Then every normal subgroup of $\Gamma$ is trivial or of finite index.

A typical example of such a group is $\operatorname{PSL}(n, \mathbb{Z}) \subset \operatorname{PSL}(n, \mathbb{R})$ for $n \geq 3$.

Remark 4.1.2. Margulis' Normal Subgroup Theorem follows from Peterson's Character Rigidity Theorem (Theorem 3.1.8) as follows. Given a normal subgroup $N<\Gamma$, the characteristic function $\chi_{N}$ is a character on $\Gamma$ with GNS representation $\pi=\lambda_{\Gamma / N} \circ p$, where $p: \Gamma \rightarrow \Gamma / N$ is the quotient map and $\lambda_{\Gamma / N}$ is the left regular representation of $\Gamma / N$. By the Character Rigidity Theorem and decomposition of the character into extremal ones, $\pi$ is the direct sum of a type I representation and possibly the left regular representation of $\Gamma$. If it contains the left regular representation of $\Gamma$, it is injective, hence $N$ is trivial. Otherwise $L(\Gamma / N)$ is of type I which implies that $\Gamma / N$ is virtually abelian [Lüc97, Lemma 3.3] and hence amenable. Since it also has property (T), it must be finite dimensional.

Definition 4.1.3. An inclusion of von Neumann algebras $N \subset M$ is regular if the normalizer of $N$ generates $M$, i.e., $\mathcal{N}_{M}(N)^{\prime \prime}=M$.

Question 4.1.4. Is it true that, maybe under some extra conditions, if $\Gamma$ is as above and $N \subset L \Gamma$ a regular subfactor then $N=\mathbb{C}$ or $[L \Gamma: N]<\infty$ ? 
This question has probably been asked before, but we couldn't find it in the literature.

In the following we restrict our attention to the situation where the image of $\Gamma$ is not only in $\mathcal{N}_{M}(N)^{\prime \prime}$, but even in $\mathcal{N}_{M}(N)$ in order to make the question accessible to Peterson's methods from the proof of character rigidity (Theorem 3.1.8. We allow subfactors in von Neumann algebras than bit more general than $L \Gamma$.

Assumption. For the rest of this chapter let $\Gamma$ be a lattice in a simple real Lie group $G$ which has trivial center and real rank at least 2. Let $M$ be a finite factor, $N \subset M$ a subfactor and $\pi: \Gamma \rightarrow \mathcal{N}_{M}(N)$ a representation of $\Gamma$ into the normalizer of $N$ such that the action $\Gamma \curvearrowright M$ given by $\alpha_{\gamma}(x)=\pi(\gamma) x \pi\left(\gamma^{-1}\right)$ is ergodic and $M=(N \cup \pi(\Gamma))^{\prime \prime}$.

Example 4.1.5. $M=L \Gamma$ with $\lambda: \Gamma \rightarrow L \Gamma$ the left regular representation and $N \subset L \Gamma$ a subfactor which is normalized by $\lambda(\Gamma)$ is as in the assumption. $M$ is a factor and the conjugation action is ergodic because $\Gamma$ is i.c.c. (Lemma 3.1 .9 .

Remark 4.1.6. It should be possible to do everything in this chapter with the same assumptions on $\Gamma$ as in Theorem 3.1.8. We restrict ourselves to the simple real case to avoid some technicalities.

\subsection{Peterson machine with coefficients in $N$}

In this section we adjust the proof of Peterson's character rigidity theorem in [Pet14] and [Pet16] to the situation described above by putting coefficients in $N$ into it. Setting $N=\mathbb{C}$ gives back the proof of character rigidity.

We will need a bunch of subgroups, which we first define in the case of $G=\operatorname{SL}(n, \mathbb{R})$.

Example 4.2.1. For $G=\operatorname{SL}(n, \mathbb{R})$ let $P$ be the subgroup of upper triangular matrices and $V$ the subgroup of upper triangular matrices with 1 on the diagonal. Fix numbers $0=j_{0}<j_{1}<j_{2}<\cdots<j_{k}=n$. We define now subgroups consisting each of all block matrices in $\operatorname{SL}(n, \mathbb{R})$ of a certain structure:

$$
\begin{aligned}
P_{0}:=\left\{\left(\begin{array}{cccc}
A_{11} & A_{12} & \ldots & A_{1 k} \\
0 & A_{22} & \ldots & A_{2 k} \\
\vdots & \vdots & \ddots & \vdots \\
0 & 0 & \ldots & A_{k k}
\end{array}\right)\right\}, \quad V_{0}:=\left\{\left(\begin{array}{cccc}
\mathbb{1} & A_{12} & \ldots & A_{1 k} \\
0 & \mathbb{1} & \ldots & A_{2 k} \\
\vdots & \vdots & \ddots & \vdots \\
0 & 0 & \ldots & \mathbb{1}
\end{array}\right)\right\}, \\
R_{0}:=\left\{\left(\begin{array}{cccc}
A_{11} & 0 & \ldots & 0 \\
0 & A_{22} & \ldots & 0 \\
\vdots & \vdots & \ddots & \vdots \\
0 & 0 & \ldots & A_{k k}
\end{array}\right)\right\}, L_{0}:=\left\{\left(\begin{array}{cccc}
V_{11} & 0 & \ldots & 0 \\
0 & V_{22} & \ldots & 0 \\
\vdots & \vdots & \ddots & \vdots \\
0 & 0 & \ldots & V_{k k}
\end{array}\right)\right\},
\end{aligned}
$$


where $A_{i l}$ are arbitrary matrices of size $\left(j_{i}-j_{i-1}\right) \times\left(j_{l}-j_{l-1}\right), V_{i i}$ are upper triangular matrices with 1 on the diagonal and $\mathbb{1}$ is an identity matrix of fitting size.

For each of these subgroups we denote by $\bar{P}, \bar{V}$, ect. the corresponding transposed subgroup.

Definition 4.2.2. For general $G$, let $S$ be an $\mathbb{R}$-split maximal torus, $P$ a minimal parabolic subgroup containing $S$ and $V<P$ its unipotent radical. Let $\bar{P}$ be the opposite parabolic and $\bar{V}$ its unipotent radical. Let $P_{0}$ be another parabolic subgroup s.t. $P<P_{0} \lesseqgtr G$. Let $V_{0}$ be the unipotent radical of $P_{0}$ and $\overline{P_{0}}, \overline{V_{0}}$ the corresponding opposite subgroups. Let $R_{0}$ be the reductive component of $P_{0}$ containing $S$ so that $P_{0}=R_{0} \rtimes V_{0}$ and $\overline{L_{0}}=R_{0} \cap \bar{V}$. Then $\bar{V}=\overline{V_{0}} \rtimes \overline{L_{0}}$. See [Mar91, I.1.2] for the definitions.

We have the following commuting diagram:

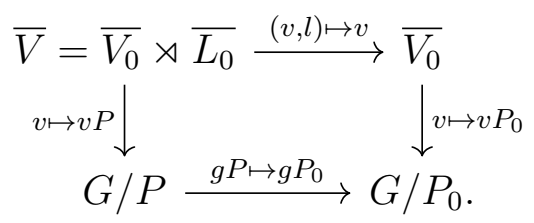

Lemma 4.2.3. The vertical maps map measures in the class of the Haar measure to G-quasiinvariant measures. They are measure isomorphisms when equipping the quotient spaces with these measures.

Proof. Let $\mu_{\bar{V}}$ be a left Haar measure on $\bar{V}$ and let $\lambda \in \mathcal{M}(G / P)$ be the image of $\mu_{\bar{V}}$. This defines by [Bou04, Proposition VII.2.1.4] a measure $\lambda^{\#}$ on $G$ given by

$$
\int_{G} f d \lambda^{\#}=\int_{G / P} \int_{P} f(g p) d \mu_{P}(p) d \lambda(g P),
$$

where $\mu_{P}$ is a left Haar measure on $P$. By [Mar91, Lemma IV.2.2], the map $\left(\bar{V} \times P, \mu_{\bar{V}} \otimes \mu_{P}\right) \rightarrow\left(G, \mu_{G}\right),(v, p) \mapsto v p^{-1}$, is a homeomorphism onto the image and a measure isomorphism, $\mu_{G}$ being a suitably normalized left Haar measure on $G$. This implies that $\lambda^{\#}=\left(1_{\bar{V}} \otimes \Delta_{P}\right) \cdot \mu_{G}$, where $\Delta_{P}$ is the modular function on $P$. It follows by [Bou04, Lemma VII.2.5.4] that $\lambda$ is $G$-quasiinvariant. Now the same follows for the images of measures that are strongly equivalent to Haar measure and analogously for such measures on $\overline{V_{0}}$.

The vertical maps are then measure isomorphisms because they map the measures to each other and are injective.

Let $\nu$ and $\rho$ be probability measures on $\overline{V_{0}}$ resp. $\overline{L_{0}}$ in the class of the Haar measure; the image of $\nu$ on $G / P_{0}$ is still denoted by $\nu$. We equip $\bar{V}=\overline{V_{0}} \rtimes \overline{L_{0}}$ with the product measure $\nu \times \rho$.

Let $G$ act on $\bar{V}$ and $\overline{V_{0}}$ in the way that makes the above diagram $G$ equivariant. This transforms the action of $\bar{V}$ on $G / P$ to left multiplication on $\bar{V}$ and the action of $R_{0}$ on $G / P$ to the action induced by conjugation on $\bar{V}$. 
Let $\sigma$ be the corresponding action of $\Gamma$ on $L^{\infty}(G / P)$ and $\sigma^{0}$ the corresponding Koopman representation on $L^{2}(G / P)$. Let

$$
P_{1}:=1 \otimes P_{\hat{1}} \in L^{\infty}(G / P) \bar{\otimes} B\left(L^{2} M\right),
$$

where $P_{\hat{1}}$ is the orthogonal projection on $\mathbb{C} \hat{1} \subset L^{2} M$ with $M$ as in the assumption. Let

$$
\mathcal{B}:=\left(L^{\infty}(G / P) \bar{\otimes} B\left(L^{2} M\right)\right) \cap\left\{\sigma_{\gamma} \otimes(J \pi(\gamma) J) \mid \gamma \in \Gamma\right\}^{\prime} \cap(1 \otimes J N J)^{\prime} .
$$

Lemma 4.2.4. There exists a conditional expectation

$$
E:\left(L^{\infty}(G / P) \bar{\otimes} B\left(L^{2} M\right)\right) \cap(1 \otimes J N J)^{\prime} \rightarrow \mathcal{B} .
$$

Proof. Let $H=L^{2}(M)$ and let $\theta: \Gamma \rightarrow \operatorname{Aut}(B(H))$ be conjugation with $J \pi(\cdot) J$. Let $\tilde{\theta}$ be the induced action of $G$ on $L^{\infty}(G / \Gamma) \bar{\otimes} B(H)$ as in Definition 1.3 .20 with a section $s: G / \Gamma \rightarrow G$ and $\chi: G \times G / \Gamma \rightarrow \Gamma$ given by $\chi(g, x)=s(g x)^{-1} g s(x) . \tilde{\theta}$ is also well-defined on $L^{\infty}(G / \Gamma) \bar{\otimes} B(H) \cap(1 \otimes J N J)^{\prime}$, which we view as bounded functions from $G / \Gamma$ to $B(H) \cap(J N J)^{\prime}$. To see this let $f \in L^{\infty}(G / \Gamma) \bar{\otimes}\left(B(H) \cap(J N J)^{\prime}\right), x \in G / \Gamma, n \in N, g \in G, \gamma=\chi\left(g, g^{-1} x\right)$ and calculate

$$
\begin{aligned}
\tilde{\theta}_{g}(f)(x) J n J & =J \pi(\gamma) J f\left(g^{-1} x\right) J \pi\left(\gamma^{-1}\right) J J n J \\
& =J \pi(\gamma) J f\left(g^{-1} x\right) J \alpha_{\gamma^{-1}}(n) J J \pi\left(\gamma^{-1}\right) J \\
& =J \pi(\gamma) J J \alpha_{\gamma^{-1}}(n) J f\left(g^{-1} x\right) J \pi\left(\gamma^{-1}\right) J \\
& =J n J J \pi(\gamma) J f\left(g^{-1} x\right) J \pi\left(\gamma^{-1}\right) J \\
& =J n J \tilde{\theta}_{g}(f)(x) .
\end{aligned}
$$

$P$ is amenable [Mar91, IV.4.4], hence [Pet16, Theorem 7.4] gives that there is a conditional expectation

$$
\begin{aligned}
E: L^{\infty}(G / \Gamma) \otimes\left(B(H) \cap(J N J)^{\prime}\right) \rightarrow & \left(L^{\infty}(G / \Gamma) \otimes\left(B(H) \cap(J N J)^{\prime}\right)\right)^{\tilde{\theta}(P)} \\
& =\left(L^{\infty}(G / \Gamma) \otimes B(H)\right)^{\tilde{\theta}(P)} \cap(1 \otimes J N J)^{\prime} .
\end{aligned}
$$

But

$$
\begin{aligned}
\left(L^{\infty}(G / \Gamma) \bar{\otimes} B(H)\right)^{\tilde{\theta}(P)} & \cong\left(L^{\infty}(G) \bar{\otimes} B(H)\right)^{(L \otimes \operatorname{id}(P)) \times(R \otimes \theta(\Gamma))} \\
& \cong\left(L^{\infty}(G) \bar{\otimes} B(H)\right)^{(R \otimes \operatorname{id}(P)) \times(L \otimes \theta(\Gamma))} \\
& \cong\left(L^{\infty}(G / P) \bar{\otimes} B(H)\right)^{\sigma \otimes \theta(\Gamma)}
\end{aligned}
$$

Here the first isomorphism is the map $\Psi$ given in Remark 1.3.21 and the second isomorphism is $f \mapsto\left(g \mapsto f\left(g^{-1}\right)\right)$. Thus $\left(L^{\infty}(G / \Gamma) \bar{\otimes} B(H)\right)^{\tilde{\theta}(P)} \cap(J N J)^{\prime}=\left(L^{\infty}(G / P) \bar{\otimes} B(H)\right)^{\sigma \otimes \theta(\Gamma)} \cap(1 \otimes J N J)^{\prime}=\mathcal{B}$. 
The following lemma is [Pet14, Lemma 4.4], only with different $\mathcal{B}$, which does not change the proof. We give it anyway in order to provide more details.

Lemma 4.2.5. Let

$$
x=x^{*} \in \mathcal{B} \subset L^{\infty}(G / P) \bar{\otimes} B\left(L^{2} M\right)=L^{\infty}\left(\overline{V_{0}}\right) \bar{\otimes} L^{\infty}\left(\overline{L_{0}}\right) \bar{\otimes} B\left(L^{2} M\right)
$$

and view it as a function from $\overline{V_{0}}$ to $L^{\infty}\left(\overline{L_{0}}\right) \bar{\otimes} B\left(L^{2} M\right)$. Let

$$
x_{0} \in L^{\infty}\left(\overline{L_{0}}\right) \bar{\otimes} B\left(L^{2} M\right)
$$

be in the SOT-essential range of $x$. Then there exists a $y=y^{*} \in \mathcal{B}$ such that $y P_{1} \in L^{\infty}\left(\overline{L_{0}}\right) \bar{\otimes} B\left(L^{2} M\right)$ and $P_{1} y P_{1}=P_{1} x_{0} P_{1}$.

Proof. That $x_{0}$ is in the SOT-essential range of $x$ means that there are subsets $E_{j} \subset \overline{V_{0}}$ of positive measure such that for all $\eta \in L^{2}(M), \xi_{L} \in L^{2}\left(\overline{L_{0}}\right)$ and $\epsilon>0$ there exists an $N$ with

$$
\int_{\overline{L_{0}}}\left\|\left(x(v, l)-x_{0}(l)\right) \eta\right\|^{2}\left|\xi_{L}(l)\right|^{2} d \rho(l)<\epsilon
$$

for all $j>N$ and all $v \in E_{j}$. By the proof of [Pet14, Lemma 4.3] there are $\gamma_{j} \in \Gamma$ and $h_{j} \in \overline{V_{0}} \rtimes \mathcal{Z}\left(R_{0}\right)$ such that $\gamma_{j} h_{j}^{-1} \rightarrow e$ and $\nu\left(h_{j} E_{j}\right) \rightarrow 1$. We first show that these can be chosen in a way that $\sigma_{\gamma_{j}}(x) \rightarrow x_{0}$ in SOT. Take a countable SOT-basis of neighborhoods of zero in the unit ball of $L^{\infty}\left(\overline{V_{0}}\right) \bar{\otimes} L^{\infty}\left(\overline{L_{0}}\right) \bar{\otimes} B\left(L^{2} M\right)$, denoted by $\left\{U_{j}\right\}_{j \in \mathbb{N}}$, such that $U_{j} \searrow\{0\}$. As the action $\sigma$ is strongly continuous, there are numbers $k(j) \in \mathbb{N}$ and neighborhoods $e \in O_{j} \subset G$ such that $O_{j} \subset O_{i}$ if $j>i$ and

$$
\sigma_{g}\left(x_{0}+U_{k(j)}\right) \subset x_{0}+U_{j} \quad \forall g \in O_{j}
$$

We can choose the $\gamma_{j}$ in [Pet14, Lemma 4.3] in a way that $\gamma_{j} h_{j}^{-1} \in O_{j}$ for all $j$. We will show now first that $\sigma_{\gamma_{j}}(x) \rightarrow x_{0}$ if $\sigma_{h_{j}}(x) \rightarrow x_{0}$ and then that $\sigma_{h_{j}}(x) \rightarrow x_{0}$, all in SOT.

So assume that $\sigma_{h_{j}}(x) \rightarrow x_{0}$, hence $\forall j \exists N: \sigma_{h_{i}}(x) \in x_{0}+U_{k(j)}$ for all $i>N$. Then by $4.2 \sigma_{\gamma_{i}}\left(x_{0}\right)=\sigma_{\gamma_{i} h_{i}^{-1}} \sigma_{h_{i}}\left(x_{0}\right) \in x_{0}+U_{j}$ for all $i>\max \{N, j\}$ because $\gamma_{i} h_{i}^{-1} \in O_{j}$. So then $\sigma_{\gamma_{j}}(x) \rightarrow x_{0}$.

To show that $\sigma_{h_{j}}(x) \rightarrow x_{0}$ let $\eta \in L^{2} M, \xi_{L} \in L^{2}\left(\overline{L_{0}}\right), \xi_{V} \in L^{2}\left(\overline{V_{0}}\right)$. Then, 
as the $h_{j} \in \overline{V_{0}} \rtimes \mathcal{Z}\left(R_{0}\right)$ act trivially on $\overline{L_{0}}$ and using 4.1 ,

$$
\begin{aligned}
& \left\|1_{h_{j} E_{j}}\left(\sigma_{h_{j}}(x)-x_{0}\right)\left(\xi_{V} \otimes \xi_{L} \otimes \eta\right)\right\|^{2} \\
= & \int_{h_{j} E_{j} \times \overline{L_{0}}}\left\|\left(\sigma_{h_{j}}(x)(v, l)-x_{0}(l)\right) \eta\right\|^{2}\left|\xi_{V}(v) \xi_{L}(l)\right|^{2} d \nu(v) d \rho(l) \\
= & \int_{h_{j} E_{j} \times \overline{L_{0}}}\left\|\left(x\left(h_{j}^{-1} v, l\right)-x_{0}(l)\right) \eta\right\|^{2}\left|\xi_{V}(v) \xi_{L}(l)\right|^{2} d \nu(v) d \rho(l) \\
= & \int_{E_{j} \times \overline{L_{0}}}\left\|\left(x(v, l)-x_{0}(l)\right) \eta\right\|^{2}\left|\xi_{V}\left(h_{j} v\right) \xi_{L}(l)\right|^{2} d\left(\left(h_{j}^{-1}\right)_{*} \nu\right)(v) d \rho(l) \\
= & \int_{E_{j}}\left(\int_{\overline{L_{0}}}\left\|\left(x(v, l)-x_{0}(l)\right) \eta\right\|^{2}\left|\xi_{L}(l)\right|^{2} d l\right)\left|\xi_{V}\left(h_{j} v\right)\right|^{2} d\left(\left(h_{j}^{-1}\right)_{*} \nu\right)(v) \\
< & \int_{h_{j} E_{j}} \epsilon\left|\xi_{V}(v)\right|^{2} d \nu(v) \leq \epsilon\left\|\xi_{V}\right\|^{2} .
\end{aligned}
$$

So $1_{h_{j} E_{j}}\left(\sigma_{h_{j}}(x)-x_{0}\right) \rightarrow 0$ in SOT, and since $\nu\left(h_{j} E_{j}\right) \rightarrow 1$, also $\sigma_{h_{j}}(x)-x_{0} \rightarrow 0$ in SOT.

Let $y$ be a WOT cluster point of the set $\left\{\pi\left(\gamma_{j}\right) x \pi\left(\gamma_{j}^{-1}\right)\right\}$. Then $y \in \mathcal{B}$ because $x \in \mathcal{B}$ and conjugation with $J N J$ and $J \pi(\Gamma) J$ commutes with conjugation with $\pi(\Gamma)$. Also $y P_{1}$ is a WOT cluster point of

$$
\begin{aligned}
\left\{\pi\left(\gamma_{j}\right) x \pi\left(\gamma_{j}^{-1}\right) P_{1}\right\} & =\left\{\pi\left(\gamma_{j}\right)\left(J \pi\left(\gamma_{j}\right) J\right)\left(J \pi\left(\gamma_{j}^{-1}\right) J\right) x\left(J \pi\left(\gamma_{j}\right) J\right) P_{1}\right\} \\
& =\left\{\pi\left(\gamma_{j}\right)\left(J \pi\left(\gamma_{j}\right) J\right) \sigma_{\gamma_{j}}(x) P_{1}\right\}
\end{aligned}
$$

Since $\sigma_{\gamma_{j}}(x) \rightarrow x_{0}$ in SOT, $y P_{1}$ must then also be a WOT cluster point of

$$
\left\{\pi\left(\gamma_{j}\right)\left(J \pi\left(\gamma_{j}\right) J\right) x_{0} P_{1}\right\} \subset L^{\infty}\left(\overline{L_{0}}\right) \bar{\otimes} B\left(L^{2} M\right),
$$

so $y P_{1} \in L^{\infty}\left(\overline{L_{0}}\right) \bar{\otimes} B\left(L^{2} M\right)$. $P_{1} y P_{1}$ is a WOT cluster point of

$$
\left\{P_{1} \pi\left(\gamma_{j}\right) x \pi\left(\gamma_{j}^{-1}\right) P_{1}\right\}=\left\{P_{1}\left(J \pi\left(\gamma_{j}\right) J\right) x\left(J \pi\left(\gamma_{j}^{-1}\right) J\right) P_{1}\right\}=\left\{P_{1} \sigma_{\gamma}(x) P_{1}\right\} .
$$

So again since $\sigma_{\gamma_{j}}(x) \rightarrow x_{0}, P_{1} y P_{1}=P_{1} x_{0} P_{1}$.

Proposition 4.2.6. If $M$ is not isomorphic to $N \rtimes \Gamma$ with isomorphism extending $\pi$, then $\mathcal{B}=M$.

Proof. Assume that $M$ is not isomorphic to $N \rtimes \Gamma$ with isomorphism extending $\pi$. Then there is a $\gamma_{0} \in \Gamma \backslash\{e\}$ and an $n \in N$ such that $c_{0}:=\tau\left(\pi\left(\gamma_{0}\right) n\right) \neq 0$ because if $\tau(n \pi(\gamma))=\tau(n) \delta_{e}(\gamma)$ for all $n \in N$ and $\gamma \in \Gamma$, the ${ }^{*}$-homomorphism $\pi(\gamma) n \mapsto(1 \otimes \lambda(\gamma)) \iota(n)$ from the ${ }^{*}$-algebra generated by $N$ and $\pi(\Gamma)$ to the *-algebra generated by $\iota(N)$ and $1 \otimes \lambda(\Gamma)$ as in Definition 1.2 .9 extends to a unitary $U: L^{2}(M, \tau) \rightarrow L^{2}\left(N \rtimes \Gamma,\left.\tau\right|_{N} \otimes \delta_{e}\right)=L^{2}(N, \tau) \otimes \ell^{2}(\Gamma)$ such that $M=U^{*}(N \rtimes \Gamma) U$.

Let $x, x_{0}$ and $y$ be as in the above lemma. We want to show that $x$ is a constant function. Let $\theta: \Gamma \rightarrow \operatorname{Aut}(M)$, different as in the proof of Lemma 
4.2.4 be conjugation by $\pi(\cdot)$ and the induced action $\tilde{\theta}: G \curvearrowright L^{\infty}(G / \Gamma) \bar{\otimes} M$ as in Definition 1.3.20.

$\theta$ is ergodic by assumption, hence $\tilde{\theta}$ is ergodic by Lemma 1.3.22. It is still ergodic when restricted to $\overline{V_{0}} \rtimes \mathcal{Z}\left(R_{0}\right)$ because $\overline{V_{0}} \rtimes \mathcal{Z}\left(R_{0}\right)$ is not compact and hence every $\overline{V_{0}} \rtimes \mathcal{Z}\left(R_{0}\right)$-invariant vector must also be $G$-invariant by the Howe-Moore property of $G$ [HM79, Theorem 5.2]. Now [Pet14, Lemma 3.2] gives us that for every neighborhood $e \in O \subset G$ and $\Gamma_{O}=\Gamma \cap O\left(\overline{V_{0}} \rtimes \mathcal{Z}\left(R_{0}\right)\right)$ we have

$$
\tau\left(\pi\left(\gamma_{0}\right) n\right)=J \tau\left(\pi\left(\gamma_{0}\right) n\right) J \in \overline{\mathrm{conv}}^{S O T}\left\{J \pi\left(\gamma^{-1}\right) \pi\left(\gamma_{0}\right) n \pi(\gamma) J \mid \gamma \in \Gamma_{O}\right\}
$$

We want to show now that $\left[\sigma_{\gamma_{0}}^{0} \otimes c_{0}, P_{1} x_{0} P_{1}\right]$ is zero. By Lemma 4.2.5 $\left[\sigma_{\gamma_{0}}^{0} \otimes c_{0}, P_{1} x_{0} P_{1}\right]=\left[\sigma_{\gamma_{0}}^{0} \otimes c_{0}, P_{1} y P_{1}\right]$. The approximation 4.3$)$ of $\tau\left(\pi\left(\gamma_{0}\right) n\right)$ gives for every $O$ an approximation

$$
\left[\sigma_{\gamma_{0}}^{0} \otimes c_{0}, P_{1} y P_{1}\right] \stackrel{S O T}{\sim} \sum_{i=1}^{k} c_{i} P_{1}\left[\sigma_{\gamma_{0}}^{0} \otimes J \pi\left(\gamma_{i}^{-1} \gamma_{0}\right) n \pi\left(\gamma_{i}\right) J, y\right] P_{1}
$$

with $\gamma_{i} \in \Gamma_{O}$ and $\sum_{i=1}^{k} c_{i}=1$.

Now write $\gamma_{i}=g_{i} h_{i}$ where $g_{i} \in O$ and $h_{i} \in \overline{V_{0}} \rtimes \mathcal{Z}\left(R_{0}\right)$. We have $\sigma_{h_{i}}\left(y P_{1}\right)=$ $y P_{1}$ and $\sigma_{h_{i}}\left(P_{1} y\right)=P_{1} y$ since $y P_{1}, P_{1} y \in L^{\infty}\left(\overline{L_{0}}\right) \bar{\otimes} B\left(L^{2} M\right)$ and taking $O$ small enough we get $\sigma_{\gamma_{i}}(y) P_{1}=\sigma_{\gamma_{i}}\left(y P_{1}\right) \sim y P_{1}$ and $P_{1} \sigma_{h_{i}}(y)=\sigma_{h_{i}}\left(P_{1} y\right) \sim P_{1} y$ in SOT. Then

$$
\begin{aligned}
& {\left[\sigma_{\gamma_{0}}^{0} \otimes c_{0}, P_{1} x_{0} P_{1}\right] } \\
& \stackrel{\mathrm{WOT}}{\sim} \sum_{i=1}^{k} c_{i} P_{1}\left[\sigma_{\gamma_{0}}^{0} \otimes J \pi\left(\gamma_{i}^{-1} \gamma_{0}\right) n \pi\left(\gamma_{i}\right) J, \sigma_{\gamma_{i}}(y)\right] P_{1} \\
&=\sum_{i=1}^{k} c_{i} P_{1}\left[\sigma_{\gamma_{0}}^{0} \otimes J \pi\left(\gamma_{i}^{-1} \gamma_{0} \gamma_{i}\right) J, \sigma_{\gamma_{i}}(y)\right]\left(J \alpha_{\gamma_{i}^{-1}}(n) J\right) P_{1} \\
&=\sum_{i=1}^{k} c_{i} P_{1}\left[\sigma_{\gamma_{0}}^{0} \otimes J \pi\left(\gamma_{i}^{-1} \gamma_{0} \gamma_{i}\right) J,\left(\sigma_{\gamma_{i}}^{0} \otimes 1\right) y\left(\sigma_{\gamma_{i}^{-1}}^{0} \otimes 1\right)\right]\left(J \alpha_{\gamma_{i}^{-1}}(n) J\right) P_{1} \\
&=\sum_{i=1}^{k} c_{i} P_{1}\left(\sigma_{\gamma_{i}}^{0} \otimes 1\right)\left[\sigma_{\gamma_{i}^{-1} \gamma_{0} \gamma_{i}}^{0} \otimes J \pi\left(\gamma_{i}^{-1} \gamma_{0} \gamma_{i}\right) J, y\right]\left(\sigma_{\gamma_{i}^{-1}}^{0} \otimes 1\right)\left(J \alpha_{\gamma_{i}^{-1}}(n) J\right) P_{1} \\
&= 0 .
\end{aligned}
$$

In the second step we used that $y$ and hence also $\sigma_{\gamma_{i}}(y)$ commutes with $J N J$. In the last step we used that $\left[\sigma_{\gamma_{i}^{-1} \gamma_{0} \gamma_{i}}^{0} \otimes J \pi\left(\gamma_{i}^{-1} \gamma_{0} \gamma_{i}\right) J, y\right]=0$ because $y \in \mathcal{B}$. So we found $\left[\sigma_{\gamma_{0}}^{0} \otimes c_{0}, P_{1} x_{0} P_{1}\right]=0$ and hence $\sigma_{\gamma_{0}}\left(P_{1} x_{0} P_{1}\right)=P_{1} x_{0} P_{1}$. Since $\tau\left(\pi\left(\gamma_{0}\right) n\right)=\tau\left(\pi(\gamma) \pi\left(\gamma_{0}\right) n \pi\left(\gamma^{-1}\right)\right)=\tau\left(\pi\left(\gamma \gamma_{0} \gamma^{-1}\right) \alpha_{\gamma}(n)\right)$, we get $\sigma_{\gamma}\left(P_{1} x_{0} P_{1}\right)=$ $P_{1} x_{0} P_{1}$ for all $\gamma \in\left\langle\left\langle\gamma_{0}\right\rangle\right\rangle$ in the normal closure of $\gamma_{0}$. By Pet16, Theorem 10.10.] the action of $\left\langle\left\langle\gamma_{0}\right\rangle\right\rangle$ on $L^{\infty}(G / P)$ is ergodic, so $P_{1} x_{0} P_{1} \in \mathbb{C} 1 \otimes P_{\hat{1}}$. Since 
$x_{0}$ was arbitrary in the range of $x$, we conclude that $P_{1} x P_{1} \in L^{\infty}\left(\overline{V_{0}}\right) \otimes P_{\hat{1}}$ and hence $P_{1} \mathcal{B} P_{1} \subset L^{\infty}\left(\overline{V_{0}}\right) \otimes P_{\hat{1}}$. This means $\mathcal{B} \subset L^{\infty}\left(\overline{V_{0}}\right) \bar{\otimes} B\left(L^{2} M\right)$ because if $x \in \mathcal{B}$ and $a, b \in M$, we have

$$
\langle x \hat{a}, \hat{b}\rangle=\left\langle\left(b^{*} x a\right) \hat{1}, \hat{1}\right\rangle=\left\langle\left(P_{1} b^{*} x a P_{1}\right) \hat{1}, \hat{1}\right\rangle \in L^{\infty}\left(\overline{V_{0}}\right)
$$

since $b^{*} x a \in \mathcal{B}$. But $\overline{V_{0}}=G / P_{0}$ and $G$ is generated by the $P_{0}$ 's Mar91, Proposition I.1.2.2], so we get

$$
\mathcal{B}=B\left(L^{2} M\right) \cap(J \pi(\Gamma) J \cup J N J)^{\prime}=M .
$$

Theorem 4.2.7. Let $\Gamma$ be a lattice in a simple real Lie group $G$ which has trivial center and real rank at least 2. Let $M$ be a finite factor, $N \subset M a$ subfactor and $\pi: \Gamma \rightarrow \mathcal{N}_{M}(N)$ a representation of $\Gamma$ into the normalizer of $N$ such that the action $\Gamma \curvearrowright M$ given by $\alpha_{\gamma}(x)=\pi(\gamma) x \pi\left(\gamma^{-1}\right)$ is ergodic and $M=(N \cup \pi(\Gamma))^{\prime \prime}$.

If $N^{\prime} \cap M$ is finite-dimensional and $M$ is not isomorphic to $N \rtimes \Gamma$ with isomorphism extending $\pi$, then $[M: N]<\infty$.

Proof. If $\pi$ does not extend to an isomorphism $M \cong N \rtimes \Gamma$, we get a conditional expectation $E: B\left(L^{2} M\right) \cap(J N J)^{\prime} \rightarrow M$ by Lemma 4.2.4 and Proposition 4.2.6. By [Pop86, 3.2.3 (ii)] this means that the inclusion $N \subset M$ is amenable. $N \subset M$ is also corigid because $\Gamma$ has property (T) [Pop86, 4.1 .7 (ii)] (note the differences in the terminology described in Remark 2.5.2). If an inclusion is amenable and corigid and $N^{\prime} \cap M$ is finite-dimensional, then the inclusion is of finite index Pop86, 4.1.8 (iv)].

Remark 4.2.8. In the case where $M=L \Gamma$ and $\pi$ is the left regular representation we have $M \cong N \rtimes \Gamma$ with isomorphism extending $\pi$ if and only if $N=\mathbb{C}$.

Remark 4.2.9. Going through the above proof or the proof of Peterson's character rigidity theorem one can see that it not only works for representations, but also for projective representations. Indeed, we only get some constants coming from the cocycle in the proof of Proposition 4.2.6, which does not change the result that the commutator is zero and the argument which follows it.

So in the case of $N=\mathbb{C}$ we get that if $\pi: \Gamma \rightarrow U(N)$ is a projective representation such that $\pi(\Gamma)^{\prime \prime}$ is a finite factor, $\pi(\Gamma)^{\prime \prime}$ is finite-dimensional or $\pi$ extends to an isomorphism $\pi(\Gamma)^{\prime \prime} \cong L \Gamma_{c}$.

\subsection{On the relative commutant}

In this section we remove the condition from Theorem 4.2.7 that the relative commutant is finite-dimensional in the case where $M=L \Gamma$ and $\pi=\lambda$. Hence we will prove the following theorem. 
Theorem 4.3.1. Let $\Gamma$ be a lattice in a simple real Lie group $G$ which has trivial center and real rank at least 2 . Let $N \subset L \Gamma$ a subfactor which is normalized by the natural copy of $\Gamma$ in $L \Gamma$. Then $N=\mathbb{C}$ or $[L \Gamma: N]<\infty$.

To prove this let, as before, $\alpha: \Gamma \rightarrow \operatorname{Aut}(N), \alpha_{\gamma}(n)=\pi(\gamma) n \pi\left(\gamma^{-1}\right)$ and

$$
\bar{\alpha}: \Gamma \rightarrow \operatorname{Out}(N)=\operatorname{Aut}(N) / \operatorname{Inn}(N), \quad \bar{\alpha}(\gamma)=[\alpha(\gamma)] .
$$

The kernel of $\bar{\alpha}$ is either trivial or of finite index by Margulis' Normal Subgroup Theorem.

Lemma 4.3.2. If the kernel of $\bar{\alpha}$ is trivial, then $M=N \rtimes \Gamma$.

Definition 4.3.3. An action $\alpha: \Gamma \curvearrowright N$ is called properly outer if $x y=\alpha_{\gamma}(y) x$ for all $y \in N$ implies $x=0$.

Proof of Lemma 4.3.2. If the kernel is trivial, $\alpha_{\gamma}$ is outer for all $\gamma \in \Gamma \backslash\{e\}$ and therefore properly outer because $N$ is a factor (see [Sun87, Definition 4.1.8 and Proposition 4.1.16], where properly outer actions are called "free"). Let $E_{N}: M \rightarrow N$ be the trace preserving conditional expectation. Then for all $y \in N$ we have

$$
E_{N}(\pi(\gamma)) y=E_{N}(\pi(\gamma) y)=E_{N}\left(\alpha_{\gamma}(y) \pi(\gamma)\right)=\alpha_{\gamma}(y) E_{N}(\pi(\gamma))
$$

which implies $E_{N}(\pi(\gamma))=0$. Hence $\tau(\pi(\gamma) x)=\tau\left(E_{N}(\pi(\gamma) x)\right)=\delta_{e}(\gamma) \tau(x)$, which implies $M=N \rtimes \Gamma$.

If the kernel is of finite index, let $\Gamma_{0}=\operatorname{ker}(\bar{\alpha})$ so that $\Gamma_{0}$ acts by inner automorphisms, hence for every $\gamma \in \Gamma_{0}$ there is a $u_{\gamma} \in U(N)$ such that for all $n \in N$

$$
\pi(\gamma) n \pi\left(\gamma^{-1}\right)=u_{\gamma} n u_{\gamma^{-1}}
$$

For $\gamma=e$ we pick the $u_{e}=1$. Define $c\left(\gamma, \gamma^{\prime}\right)=u_{\gamma \gamma^{\prime}} u_{\gamma^{\prime}}^{-1} u_{\gamma}^{-1}$ for all $\gamma, \gamma^{\prime} \in \Gamma$. Then $c\left(\gamma, \gamma^{\prime}\right) \in U(\mathcal{Z}(N))=S^{1}$ because $N$ is a factor, and $c$ is a 2-cocycle. Now

$$
\rho: \Gamma_{0} \rightarrow U\left(N^{\prime} \cap M\right), \quad \gamma \mapsto \pi(\gamma) u_{\gamma}^{-1},
$$

takes values in $N^{\prime} \cap M$ because of (4.4) and we have

$$
\begin{aligned}
\rho(g h) & =\pi(g h) u_{g h}^{-1}=\pi(g) \pi(h)\left(c(g, h) u_{g} u_{h}\right)^{-1}=c(g, h)^{-1} \pi(g) \pi(h) u_{h}^{-1} u_{g}^{-1} \\
& =c(g, h)^{-1} \pi(g) u_{g}^{-1} \pi(h) u_{h}^{-1}=c(g, h)^{-1} \rho(g) \rho(h) .
\end{aligned}
$$

Hence we got two projective representations, $\rho: \Gamma_{0} \rightarrow N^{\prime} \cap M$ with cocycle $c^{-1}$ and $u: \Gamma_{0} \rightarrow N$ with cocycle $c$. Up to here we did not use $M=L \Gamma$ and $\pi=\lambda$. 
Lemma 4.3.4. $u\left(\Gamma_{0}\right)^{\prime \prime}$ and $\rho\left(\Gamma_{0}\right)^{\prime \prime}$ both have finite center and we have

$$
\operatorname{dim}_{\rho\left(\Gamma_{0}\right)^{\prime \prime}} L^{2}\left(N^{\prime} \cap M\right)<\infty \text { and } \operatorname{dim}_{u\left(\Gamma_{0}\right)^{\prime \prime}} L^{2}(N)<\infty .
$$

Proof. By [Cam07, Lemma IV.3 and Lemma IV.5], we have $\tau(x y)=\tau(x) \tau(y)$ for all $x \in N^{\prime} \cap M$ and $y \in N$ and

$$
M \supset\left(\left(N^{\prime} \cap M\right) \cup N\right)^{\prime \prime} \cong\left(N^{\prime} \cap M\right) \bar{\otimes} N
$$

Let $F \subset \Gamma$ be a finite subset such that $\Gamma=\left\langle\Gamma_{0}, F\right\rangle$. Let

$$
M_{0}:=\lambda\left(\Gamma_{0}\right)^{\prime \prime}=(\rho \otimes u)\left(\Gamma_{0}\right)^{\prime \prime} \subset \rho\left(\Gamma_{0}\right)^{\prime \prime} \bar{\otimes} u\left(\Gamma_{0}\right)^{\prime \prime} \subset\left(N^{\prime} \cap M\right) \bar{\otimes} N .
$$

Since $M=\lambda(\Gamma)^{\prime \prime}$, we have

$$
M=\sum_{\gamma \in F} M_{0} \lambda(\gamma)=\sum_{\gamma \in F}\left(\left(N^{\prime} \cap M\right) \bar{\otimes} N\right) \lambda(\gamma) .
$$

Now the claims about dimensions follow from the following estimate using Lemma 1.2 .27

$$
\begin{aligned}
\infty>\operatorname{dim}_{M_{0}}\left(L^{2} M\right) & \geq \operatorname{dim}_{(\rho \otimes u)\left(\Gamma_{0}\right)^{\prime \prime}}\left(L^{2}\left(\left(N^{\prime} \cap M\right) \bar{\otimes} N\right)\right) \\
& \geq \operatorname{dim}_{\rho\left(\Gamma_{0}\right)^{\prime \prime} \bar{\otimes} u\left(\Gamma_{0}\right)^{\prime \prime}}\left(L^{2}\left(\left(N^{\prime} \cap M\right) \bar{\otimes} N\right)\right) \\
& =\operatorname{dim}_{\rho\left(\Gamma_{0}\right)^{\prime \prime}}\left(L^{2}\left(N^{\prime} \cap M\right)\right) \cdot \operatorname{dim}_{u\left(\Gamma_{0}\right)^{\prime \prime}}\left(L^{2}(N)\right) .
\end{aligned}
$$

$\Gamma / \Gamma_{0}$ acts on $\mathcal{Z}\left(\rho\left(\Gamma_{0}\right)^{\prime \prime}\right)$ and $\mathcal{Z}\left(u\left(\Gamma_{0}\right)^{\prime \prime}\right)$ by conjugation with $\lambda$ because $\left.\lambda\right|_{\Gamma_{0}}=$ $\rho \otimes u$ and $\lambda(\Gamma)$ normalizes $N$ and $N^{\prime} \cap M$. Since $M$ is a factor, these actions must be ergodic. As $\Gamma / \Gamma_{0}$ is finite, $\mathcal{Z}\left(\rho\left(\Gamma_{0}\right)^{\prime \prime}\right)$ and $\mathcal{Z}\left(u\left(\Gamma_{0}\right)^{\prime \prime}\right)$ must also be finite.

Lemma 4.3.5. $\rho$ and $u$ generate finite-dimensional von Neumann algebras or are the direct sum of a projective representation (with cocycle $c$ resp. $c^{-1}$ ) generating a finite-dimensional von Neumann algebra and a twisted left regular representation (with cocycle c resp. $c^{-1}$ ).

Proof. We want to use Remark 4.2 .9 for $u$ and $\rho$. Since the center of $u\left(\Gamma_{0}\right)^{\prime \prime}$ is finite, we can decompose it into a finite direct sum of factors, $u\left(\Gamma_{0}\right)^{\prime \prime}=\bigoplus N_{i}$ and $u=\bigoplus u_{i}$ decomposes into a direct sum of projective representations such that $u_{i}\left(\Gamma_{0}\right)^{\prime \prime}=N_{i}$. Then the claim follows for $u$ and analogously for $\rho$.

So both $u$ and $\rho$ have a finite-dimensional and possibly a left regular component. The following lemma shows that they can't simultaneously have a left regular component.

Lemma 4.3.6. It cannot happen that $\rho=\rho^{\mathrm{fin}} \oplus \lambda_{c^{-1}}$ and $u=u^{\mathrm{fin}} \oplus \lambda_{c}$ such that $\rho^{\mathrm{fin}}\left(\Gamma_{0}\right)^{\prime \prime}$ and $u^{\mathrm{fin}}\left(\Gamma_{0}\right)^{\prime \prime}$ are finite-dimensional. 
Proof. Assume that $\rho$ and $u$ are as above such that $B$ is a basis of $\rho^{\text {fin }}\left(\Gamma_{0}\right)^{\prime \prime}$ and $C$ is a basis $u^{\mathrm{fin}}\left(\Gamma_{0}\right)^{\prime \prime}$. Recall (4.5) and let $E: M \rightarrow\left(N^{\prime} \cap M\right) \bar{\otimes} N$ be the trace-preserving conditional expectation. Then 4.6 implies

$$
\begin{aligned}
\left(N^{\prime} \cap M\right) \bar{\otimes} N & =E(M)=E\left(\sum_{\gamma \in F} M_{0} \lambda(\gamma)\right)=\sum_{\gamma \in F} M_{0} E(\lambda(\gamma)) \\
& =\sum_{\gamma \in F}\left(\left(\rho^{\text {fin }} \oplus \lambda_{c^{-1}}\right) \otimes\left(u^{\text {fin }} \oplus \lambda_{c}\right)\right)\left(\Gamma_{0}\right)^{\prime \prime} E(\lambda(\gamma)) \\
& =\sum_{\gamma \in F} \sum_{x \in B} \sum_{y \in C}\left(\lambda_{c^{-1}} \otimes \lambda_{c}\right)\left(\Gamma_{0}\right)^{\prime \prime} x y E(\lambda(\gamma))
\end{aligned}
$$

The left hand side contains $\lambda_{c^{-1}}\left(\Gamma_{0}\right)^{\prime \prime} \bar{\otimes} \lambda_{c}\left(\Gamma_{0}\right)^{\prime \prime}$ and the right hand side is finitedimensional over $\left(\lambda_{c^{-1}} \otimes \lambda_{c}\right)\left(\Gamma_{0}\right)^{\prime \prime}$. To bring this to a contradiction to Lemma 1.2 .27 we show that

$$
\operatorname{dim}_{\left(\lambda_{c^{-1}} \otimes \lambda_{c}\right)\left(\Gamma_{0}\right)^{\prime \prime}} L^{2}\left(\lambda_{c^{-1}}\left(\Gamma_{0}\right)^{\prime \prime} \bar{\otimes} \lambda_{c}\left(\Gamma_{0}\right)^{\prime \prime}\right)=\infty .
$$

For this we prove that if $g, h, g^{\prime}, h^{\prime} \in \Gamma_{0}$ such that $h^{-1} g \neq h^{\prime-1} g^{\prime}$, the sets

$$
\left(\lambda_{c^{-1}} \otimes \lambda_{c}\right)\left(\Gamma_{0}\right)^{\prime \prime}\left(\lambda_{c^{-1}}(g) \otimes \lambda_{c}(h)\right) \quad \text { and } \quad\left(\lambda_{c^{-1}} \otimes \lambda_{c}\right)\left(\Gamma_{0}\right)^{\prime \prime}\left(\lambda_{c^{-1}}\left(g^{\prime}\right) \otimes \lambda_{c}\left(h^{\prime}\right)\right)
$$

are orthogonal in $L^{2}\left(\lambda_{c^{-1}}\left(\Gamma_{0}\right)^{\prime \prime} \bar{\otimes} \lambda_{c}\left(\Gamma_{0}\right)^{\prime \prime}\right)$. Indeed, for each $\gamma, \gamma^{\prime} \in \Gamma_{0}$,

$$
\begin{aligned}
& \left\langle\left(\lambda_{c^{-1}} \otimes \lambda_{c}\right)(\gamma)\left(\lambda_{c^{-1}}(g) \otimes \lambda_{c}(h)\right),\left(\lambda_{c^{-1}} \otimes \lambda_{c}\right)\left(\gamma^{\prime}\right)\left(\lambda_{c^{-1}}\left(g^{\prime}\right) \otimes \lambda_{c}\left(h^{\prime}\right)\right)\right\rangle \\
& =\left\langle\lambda_{c^{-1}}(\gamma) \lambda_{c^{-1}}(g), \lambda_{c^{-1}}\left(\gamma^{\prime}\right) \lambda_{c^{-1}}\left(g^{\prime}\right)\right\rangle \cdot\left\langle\lambda_{c}(\gamma) \lambda_{c}(h), \lambda_{c}\left(\gamma^{\prime}\right) \lambda_{c}\left(h^{\prime}\right)\right\rangle .
\end{aligned}
$$

The first factor is nonzero iff $g g^{\prime-1}=\gamma^{-1} \gamma^{\prime}$, the second factor is nonzero iff $h h^{\prime-1}=\gamma^{-1} \gamma^{\prime}$. So if the product is nonzero, $g g^{\prime-1}=h h^{\prime-1}$. Hence $h^{-1} g=$ $h^{\prime-1} g^{\prime}$, contradicting the assumption.

Hence $u\left(\Gamma_{0}\right)^{\prime \prime}$ is finite-dimensional or $\rho\left(\Gamma_{0}\right)^{\prime \prime}$ is finite-dimensional. If $u\left(\Gamma_{0}\right)^{\prime \prime}$ is finite-dimensional, then $N$ is also finite-dimensional because $\operatorname{dim}_{u\left(\Gamma_{0}\right)^{\prime \prime}} L^{2}(N)$ is finite, which implies $N=\mathbb{C}$ by Lemma 3.5.5. If $\rho\left(\Gamma_{0}\right)^{\prime \prime}$ is finite-dimensional, then $N^{\prime} \cap M$ is finite-dimensional since $\operatorname{dim}_{\rho\left(\Gamma_{0}\right)^{\prime \prime}} L^{2}\left(N^{\prime} \cap M\right)$ is finite. This proves Theorem 4.3.1

Remark 4.3.7. Another approach to obtain results as in this chapter would be to study the character $\gamma \mapsto\left\|E_{N}(\pi(\gamma))\right\|_{2}^{2}$ and use character rigidity of $\Gamma$. 


\section{Bibliography}

[AD87] Claire Anantharaman-Delaroche. On Connes' property $T$ for von Neumann algebras. Math. Japon., 32(3):337-355, 1987.

[AD05] Claire Anantharaman-Delaroche. Cohomology of property $T$ groupoids and applications. Ergodic Theory Dynam. Systems, 25(4):977-1013, 2005.

[ADP10] Claire Anantharaman-Delaroche and Sorin Popa. An introduction to $I I_{1}$ factors, preliminary version. http://www . univ-orleans.fr/ mapmo/membres/anantharaman/, 2010.

[ADR00] Claire Anantharaman-Delaroche and Jean Renault. Amenable groupoids, volume 36 of Monographies de L'Enseignement Mathématique [Monographs of L'Enseignement Mathématique]. L'Enseignement Mathématique, Geneva, 2000.

[AGV14] Miklós Abért, Yair Glasner, and Bálint Virág. Kesten's theorem for invariant random subgroups. Duke Math. J., 163(3):465-488, 2014.

[Bla06] Bruce Blackadar. Operator algebras, volume 122 of Encyclopaedia of Mathematical Sciences. Springer-Verlag, Berlin, 2006. Theory of $C^{*}$-algebras and von Neumann algebras, Operator Algebras and Non-commutative Geometry, III.

[Bou04] Nicolas Bourbaki. Integration. II. Chapters 7-9. Elements of Mathematics (Berlin). Springer-Verlag, Berlin, 2004.

[Cam07] Jan M. Cameron. Normalizers of finite von Neumann algebras. Texas University, Dissertation, 2007.

[CJ85] Alain Connes and Vaughan Jones. Property $T$ for von Neumann algebras. Bull. London Math. Soc., 17(1):57-62, 1985.

[CSU16] Ionut Chifan, Thomas Sinclair, and Bogdan Udrea. Inner amenability for groups and central sequences in factors. Ergodic Theory Dynam. Systems, 36(4):1106-1129, 2016. 
[Dix81] Jacques Dixmier. von Neumann algebras, volume 27 of NorthHolland Mathematical Library. North-Holland Publishing Co., Amsterdam-New York, 1981.

[Gla03] Eli Glasner. Ergodic theory via joinings, volume 101 of Mathematical Surveys and Monographs. American Mathematical Society, Providence, RI, 2003.

[HM79] Roger E. Howe and Calvin C. Moore. Asymptotic properties of unitary representations. J. Funct. Anal., 32(1):72-96, 1979.

[Ioa10] Adrian Ioana. Relative property $(\mathrm{T})$ for the subequivalence relations induced by the action of $\mathrm{SL}_{2}(\mathbb{Z})$ on $\mathbb{T}^{2}$. Adv. Math., 224(4):15891617, 2010.

[JS87] Vaughan F. R. Jones and Klaus Schmidt. Asymptotically invariant sequences and approximate finiteness. Amer. J. Math., 109(1):91$114,1987$.

[KM04] Alexander S. Kechris and Benjamin D. Miller. Topics in orbit equivalence, volume 1852 of Lecture Notes in Mathematics. SpringerVerlag, Berlin, 2004.

[Lüc97] Wolfgang Lück. Hilbert modules and modules over finite von Neumann algebras and applications to $L^{2}$-invariants. Math. Ann., 309:247-285, 1997.

[Lüc02] Wolfgang Lück. $L^{2}$-invariants: theory and applications to geometry and K-theory, volume 44 of Ergebnisse der Mathematik und ihrer Grenzgebiete. 3. Folge. Springer-Verlag, Berlin, 2002.

[Lup17] Martino Lupini. A von Neumann algebra characterization of property (T) for groupoids. arxiv. org/pdf/1708. 01763. pdf, 2017. preprint.

[Mar91] Grigory A. Margulis. Discrete subgroups of semisimple Lie groups, volume 17 of Ergebnisse der Mathematik und ihrer Grenzgebiete (3). Springer-Verlag, Berlin, 1991.

[Moo82] Calvin C. Moore. Ergodic theory and von Neumann algebras. In Operator algebras and applications, Part 2 (Kingston, Ont., 1980), volume 38 of Proc. Sympos. Pure Math., pages 179-226. Amer. Math. Soc., Providence, R.I., 1982.

[Par67] T. R. Parthasarathy. Probability measures on metric spaces. Probability and Mathematical Statistics: A Series of Monographs and Textbooks. Academic Press, New York, 1967. 
[Pet14] Jesse Peterson. Character rigidity for lattices in higher-rank groups. www. math. vanderbilt. edu/ peters10/rigidity.pdf, 2014. preprint.

[Pet16] Jesse Peterson. Lecture notes on "Character Rigidity". www. math. vanderbilt. edu/peters10/notes. html, 2016.

[Pop86] Sorin Popa. Correspondences. www. math.ucla. edu/ popa/ popa-correspondences. pdf, 1986. preprint.

[Pop06] Sorin Popa. On a class of type $\mathrm{II}_{1}$ factors with Betti numbers invariants. Ann. of Math. (2), 163(3):809-899, 2006.

[PT16] Jesse Peterson and Andreas Thom. Character rigidity for special linear groups. J. reine u. angew. Math., 716:207-228, 2016.

[Sch81] Klaus Schmidt. Amenability, Kazhdan's property T, strong ergodicity and invariant means for ergodic group-actions. Ergodic Theory Dynamical Systems, 1(2):223-236, 1981.

[ST10] Roman Sauer and Andreas Thom. A spectral sequence to compute $L^{2}$-Betti numbers of groups and groupoids. J. Lond. Math. Soc. (2), 81(3):747-773, 2010.

[Str81] Şerban Strătilă. Modular theory in operator algebras. Editura Academiei Republicii Socialiste România, Bucharest; Abacus Press, Tunbridge Wells, 1981.

[Sun87] Vaikalathur S. Sunder. An invitation to von Neumann algebras. Universitext. Springer-Verlag, New York, 1987.

[SZ94] Garrett Stuck and Robert J. Zimmer. Stabilizers for ergodic actions of higher rank semisimple groups. Ann. of Math. (2), 139(3):723$747,1994$.

[Tak02] Masamichi Takesaki. Theory of operator algebras. I, volume 124 of Encyclopaedia of Mathematical Sciences. Springer-Verlag, Berlin, 2002. Operator Algebras and Non-commutative Geometry, 5.

[Tak03] Masamichi Takesaki. Theory of operator algebras. II, volume 125 of Encyclopaedia of Mathematical Sciences. Springer-Verlag, Berlin, 2003. Operator Algebras and Non-commutative Geometry, 6.

[Tho64a] Elmar Thoma. Die unzerlegbaren, positiv-definiten Klassenfunktionen der abzählbar unendlichen, symmetrischen Gruppe. Math. Z., 85:40-61, 1964. 
[Tho64b] Elmar Thoma. Über unitäre Darstellungen abzählbarer, diskreter Gruppen. Math. Ann., 153:111-138, 1964.

[Vae07] Stefaan Vaes. Rigidity results for Bernoulli actions and their von Neumann algebras (after Sorin Popa). Astérisque, (311):Exp. No. 961, viii, 237-294, 2007. Séminaire Bourbaki. Vol. 2005/2006.

[Ver10] Anatoly Vershik. Nonfree actions of countable groups and their characters. Zap. Nauchn. Sem. S.-Peterburg. Otdel. Mat. Inst. Steklov. (POMI), 378(Teoriya Predstavleniı̌, Dinamicheskie Sistemy, Kombinatornye Metody. XVIII):5-16, 228, 2010.

[VK81] Anatoly Vershik and Sergei Kerov. Characters and factor representations of the infinite symmetric group. Dokl. Akad. Nauk SSSR, 257(5):1037-1040, 1981.

[Zim81] Robert J. Zimmer. On the cohomology of ergodic actions of semisimple Lie groups and discrete subgroups. Amer. J. Math., 103(5):937951, 1981. 